\title{
MULTIPERMUTATION SOLUTIONS OF THE YANG-BAXTER EQUATION
}

\author{
TATIANA GATEVA-IVANOVA AND PETER CAMERON
}

\begin{abstract}
Set-theoretic solutions of the Yang-Baxter equation form a meetingground of mathematical physics, algebra and combinatorics. Such a solution consists of a set $X$ and a function $r: X \times X \rightarrow X \times X$ which satisfies the braid relation. We examine solutions here mainly from the point of view of finite permutation groups: a solution gives rise to a map from $X$ to the symmetric group $\operatorname{Sym}(X)$ on $X$ satisfying certain conditions.

Our results include many new constructions based on strong twisted union and wreath product, with an investigation of retracts and the multipermutation level and the solvable length of the groups defined by the solutions; and new results about decompositions and factorisations of the groups defined by invariant subsets of the solution.
\end{abstract}

\section{Contents}

1. Introduction

2. Preliminaries on set-theoretic solutions 4

3. Homomorphisms, automorphisms, strong twisted unions 11

4. Decomposition of solutions. 16

5. Multipermutation solutions of low levels. 23

6. Solutions with abelian permutation group 30

7. Multipermutation solutions of finite multipermutation level 36

8. Wreath products of solutions 46

9. Infinite solutions 52

10. More about YB permutation groups 58

References $\quad 59$

Date: October 25, 2018.

The first author is partially supported by Isaac Newton Institute, UK, The Abdus Salam International Centre for Theoretical Physics (ICTP), and by Grant MI 1503/2005 of the Bulgarian National Science Fund of the Ministry of Education and Science. 


\section{INTRODUCTION}

Let $V$ be a vector space over a field $k$. It is well-known that the "Yang-Baxter equations" on a linear map $R: V \otimes V \rightarrow V \otimes V$, the equation

$$
R_{12} R_{23} R_{12}=R_{23} R_{12} R_{23}
$$

(where $R_{i, j}$ denotes $R$ acting in the $i, j$ place in $V \otimes V \otimes V$ ), give rise to a linear representation of the braid group on tensor powers of $V$. When $R^{2}=$ id one says that the solution is involutive, and in this case one has a representation of the symmetric group on tensor powers.

A particularly nice class of solutions is provided by set-theoretic solutions where $X$ is a set and $r: X \times X \rightarrow X \times X$ obeys similar relations on $X \times X \times X$. Of course, each such solution extends linearly to $V=k X$ with matrices in this natural basis having only entries from 0,1 and many other nice properties.

Associated to each set-theoretic solution are several algebraic constructions: the semigroup $S(X, r)$, the group $G(x, r)$, the semigroup algebra $k S(X, r)=k_{R}[V]$ generated by $X$ with relations $x y=r(x, y)$ (where $\cdot$ denotes product in the free semigroup, resp free group) and the permutation group $\mathcal{G}(X, r) \subset \operatorname{Sym}(X)$ defined by the corresponding left translations $y \mapsto{ }^{x} y$ for $x \in X$, where $r(x, y)=\left({ }^{x} y, y^{x}\right)$ (under assumptions which will be given later).

In this paper we study the special case when $(X, r)$ is a square-free solution, a square-free symmetric set of arbitrary cardinality. Our special interest is in the retractability of such solutions. We study multipermutation solutions and find close relation between the multipermutation level of such a solution and the properties of the associated algebraic objects $\mathcal{G}(X, r), G(X, r)$, and $S(X, r)$. A feature of our approach is to give prominence to the group $\mathcal{G}(X, r)$.

We introduce and study various constructions of solutions such as strong twisted unions of solutions, doubling of solutions, wreath product of solutions, and various other constructions, and study the multipermutation level of the new solutions and properties such as solvable length of their associated algebraic structures.

We now describe the contents of the paper in greater detail.

In Section 2 we recall basic definitions and basic results, and give a general description of the permutation groups $H \subseteq \operatorname{Sym}(X)$ which can serve as a YB permutation group of some square-free solution $(X, r)$, see Proposition 2.17

In Section 3 we recall the definitions and basic properties of homomorphisms and automorphisms of solutions, we give a general construction, the strong twisted union of a finite number of solutions, and describe strong twisted unions in terms of split maps, see Proposition 3.12

In Section 4 we study various decompositions of square-free solutions $(X, r)$ into disjoint unions of a finite number of $r$-invariant subsets and the corresponding factorisation of $S(X, r), G(X, r)$, and $\mathcal{G}(X, r)$, see Theorems 4.16, 4.19] and 4.21] We make essential use of the matched pairs approach to solutions (in the most general setting), developed in GIM08.

Section 5 continues the study of multipermutation solutions of low levels which was initiated in GIM07, and deepened in GIM0806] with detailed description of 
solutions of multipermutation level 2. Using matched pair approach and results from GIM08, we show in Proposition 5.6 that when $(X, r)$ has multipermutation level 2, the associated braided monoid $\left(S, r_{S}\right)$ and the associated braided group $\left(G, r_{G}\right)$ are symmetric sets which inherit some nice combinatorial conditions such as the cyclic condition and condition lri (seeDefinition 2.9) but they are not squarefree. Furthermore, $S$ (respectively $G$ ) acts on itself as automorphisms. Proposition 5.8 characterises the permutation groups $H \subset \operatorname{Sym}(X)$ which define (via the left action) square-free solutions $(X, r)$ with multipermutation level 2. As a corollary we obtain that every finite abelian group $H$ is isomorphic to the permutation group $\mathcal{G}(X, r)$ of some square-free solution with multipermutation level 2.

We characterise solutions with multipermutation level 3 in Proposition 5.12, and Corollary 5.13 . We show that, for $(X, r)$ of arbitrary cardinality, but having only a finite number of $G$-orbits, each solution with multipermutation level 3 decomposes as a strong twisted union of a finite number of solutions with multipermutation level $\leq 2$, and the permutation group $\mathcal{G}$ decomposes as a product of abelian subgroups, see Propositions 5.15, and 5.12

One can say a surprising amount about solutions $(X, r)$ for which $\mathcal{G}(X, r)$ is abelian: this is the theme of Section [6. In Subsection 6.1 we develop a technique for computation with actions in the case of abelian $\mathcal{G}$.

This technique and Theorem 7.10 are used to prove the main results of the section: Theorems 6.1 and 6.3. We assume $(X, r)$ is a square-free solution of arbitrary cardinality, $\mathcal{G}=\mathcal{G}(X, r)$ is abelian, and $X$ has finite number $t$ of $\mathcal{G}$-orbits. Theorems 6.1 shows that every such a solution is a multipermutation solution of level mpl $X \leq$ $t$, and $X$ decomposes as a strong twisted union of its orbits. Furthermore, each orbit $X_{i}$ is itself a trivial solution (so $m p l X_{i}=1$ )). This confirms two conjectures of the first author (only for the case of abelian $\mathcal{G}$, of course), see GI Conjecture I, Conjecture II, formulated for finite square-free solutions (see also Conjecture 2.26).

Theorem 6.3 proves that, under the assumption that $\mathcal{G}(X, r)$ is abelian, a strong twisted union $X=X_{1} \natural X_{2}$ of two multipermutation solutions is itself a multipermutation solution with $\operatorname{mpl} X \leq \operatorname{mpl} X_{1}+\operatorname{mpl} X_{2}$. In the general case, the question whether a strong twisted union $X=X_{1} \curvearrowleft X_{2}$ of multipermutation solutions $X_{1}, X_{2}$ is also a multipermutation solution remains open.

A resent result of Cedó, Jespers, and Okniński, CJR09, shows that each finite square-free solution $(X, r)$ with $\mathcal{G}$ abelian is retractable. The proof is combinatorial (and different from ours) and relies strongly on the finiteness of $X$; there is no estimate of mpl $X$. Our proof uses a general technique which is applicable to solutions with arbitrary cardinality of $X$ (but a finite number $t$ of $G$-orbits). In fact we show that $t$ is an upper bound for the multipermutation level $\operatorname{mpl} X$.

Section 7 studies the general case of multipermutation square-free solutions. In Subsection [7.1 we recall basic notions and facts from [GI], and use them to develop a basic technique for dealing with retracts and retract classes. Theorem 7.10 gives an explicit identity in terms of action necessary and sufficient for $m p l X=m$. This identity plays an essential role in the paper.

Subsection 7.2 contains some of the important results of this paper. We study the groups $G=G(X . r)$ and $\mathcal{G}=\mathcal{G}(X, r)$ of multipermutation solutions. We show that 
if $(X, r)$ is a square-free multipermutation solution (of arbitrary cardinality) the groups $G$ and $\mathcal{G}$ are solvable. This was known for finite symmetric sets, see [ESS, (see also GI] for finite square-free solutions), but no information about the solvable length of $G$ was known.

Lemma 7.15 and induction on $m=\operatorname{mpl} X$ allow us not only to prove solvability (without assuming $X$ or $\mathcal{G}$ finite), but also to find an upper bound for the solvable lengths: $\operatorname{sl} G \leq m$ and $\operatorname{sl} \mathcal{G} \leq m-1$. The results of Section 9 show that these upper bounds are attained. Theorem 7.25 verifies that, whenever $(X, r)$ is a square-free solution of finite order, then $\operatorname{sl}(G)=\operatorname{sl}(\mathcal{G})+1$.

In Section 8 we define the notion of wreath product of solutions, by analogy with the wreath products of permutation groups. Theorem 8.7 shows that wreath product $(Z, r)=\left(X_{0}, r_{X_{0}}\right)$ wr $\left(Y, r_{Y}\right)$ is a square-free solution, with $\mathcal{G}(Z, r)=$ $\mathcal{G}\left(X_{0}, r_{X_{0}}\right)$ wr $\mathcal{G}\left(Y, r_{Y}\right)$. Furthermore, whenever $\left(X_{0}, r_{X_{0}}\right)$ and $\left(Y, r_{Y}\right)$ are multipermutation solutions, one has $\operatorname{mpl}(Z, r)=\operatorname{mpl}\left(X_{0}, r_{X_{0}}\right)+\operatorname{mpl}\left(Y, r_{Y}\right)-1$.

In Section 9 we construct an interesting sequence of explicitly defined solutions $\left(X_{m}, r_{m}\right), m=0,1,2, \ldots$, such that $\operatorname{mpl}\left(X_{m}\right)=m, \operatorname{Ret}\left(X_{m+1}, r_{m+1}\right) \simeq\left(X_{m}, r_{m}\right)$, $m=\operatorname{sl} G\left(X, r_{m}\right)=\operatorname{sl} \mathcal{G}\left(X, r_{m}\right)+1$ : see Definition 9.9 and Theorem 9.11.

\section{Preliminaries on Set-Theoretic SOlutions}

There are many works on set-theoretic solutions and related structures, of which a relevant selection for the interested reader is WX, GB, ESS, GI, GI04, GIM07, GIM08, GIM0806, LYZ, $\mathrm{Ru}, \mathrm{Ta}, \mathrm{V}$. In this section we recall basic notions and results which will be used in the paper. We shall use the terminology, notation and some results from GI, GIM07, GIM08, GIM0806.

Definition 2.1. Let $X$ be a nonempty set (not necessarily finite) and let $r$ : $X \times X \longrightarrow X \times X$ be a bijective map. We refer to it as a quadratic set, and denote it by $(X, r)$. The image of $(x, y)$ under $r$ is presented as

$$
r(x, y)=\left({ }^{x} y, x^{y}\right) .
$$

The formula (2.1) defines a "left action" $\mathcal{L}: X \times X \longrightarrow X$, and a "right action" $\mathcal{R}: X \times X \longrightarrow X$, on $X$ as:

$$
\mathcal{L}_{x}(y)={ }^{x} y, \quad \mathcal{R}_{y}(x)=x^{y},
$$

for all $x, y \in X$. The map $r$ is nondegenerate, if the maps $\mathcal{L}_{x}$ and $\mathcal{R}_{x}$ are bijective for each $x \in X$. In this paper we shall consider only the case where $r$ is nondegenerate. As a notational tool, we shall often identify the sets $X \times X$ and $X^{2}$, the set of all monomials of length two in the free semigroup $\langle X\rangle$.

Definition 2.2. (1) $r$ is square-free if $r(x, x)=(x, x)$ for all $x \in X$.

(2) $r$ is a set-theoretic solution of the Yang-Baxter equation or, shortly a solution (YBE) if the braid relation

$$
r^{12} r^{23} r^{12}=r^{23} r^{12} r^{23}
$$

holds in $X \times X \times X$, where the two bijective maps $r^{i i+1}: X^{3} \longrightarrow X^{3}$, $1 \leq i \leq 2$ are defined as $r^{12}=r \times \mathrm{id}_{X}$, and $r^{23}=\mathrm{id}_{X} \times r$. In this case we refer to $(X, r)$ also as a braided set.

(3) A braided set $(X, r)$ with $r$ involutive is called a symmetric set. 
Convention 2.3. By square-free solution we mean a nondegenerate square-free symmetric set $(X, r)$, where $X$ is a set of arbitrary cardinality. Alternative definitions are given in terms of the left action, see Lemma 2.12 and Corollary 2.14.

To each quadratic set $(X, r)$ we associate canonical algebraic objects generated by $X$ and with quadratic defining relations $\Re=\Re(r)$ defined by

$$
x y=z t \in \Re(r), \quad \text { whenever } \quad r(x, y)=(z, t) .
$$

Definition 2.4. Let $(X, r)$ be a quadratic set.

(i) The unital semigroup $S=S(X, r)=\langle X ; \Re(r)\rangle$, with a set of generators $X$ and a set of defining relations $\Re(r)$, is called the semigroup associated with $(X, r)$.

(ii) The group $G=G(X, r)$ associated with $(X, r)$ is defined as $G=G(X, r)=$ ${ }_{g r}\langle X ; \Re(r)\rangle$

(iii) For arbitrary fixed field $k$, the $k$-algebra associated with $(X, r)$ is defined as $\mathcal{A}(k, X, r)=k\langle X ; \Re(r)\rangle$. $(\mathcal{A}(k, X, r)$ is isomorphic to the monoidal algebra $k S(X, r))$.

(iv) To each nondegenerate braided set $(X, r)$ we also associate a permutation group, called the group of left action and denoted $\mathcal{G}=\mathcal{G}(X, r)$, see Definition 2.7.

If $(X, r)$ is a solution, then $S(X, r)$, resp. $G(X, r)$, resp. $\mathcal{G}(X, r)$, resp. $\mathcal{A}(k, X, r)$ is called the Yang-Baxter semigroup, resp. the Yang-Baxter group, , resp. the Yang-Baxter algebra, resp. the (Yang-Baxter) permutation group or shortly the $Y B$ permutation group, associated to $(X, r)$

The YB permutation group $\mathcal{G}(X, r)$ will be of particular importance in this paper.

Example 2.5. For arbitrary nonempty set $X$, denote by $\tau_{X}=\tau$ the flip map $\tau(x, y)=(y, x)$ for all $x, y \in X$. Then $(X, \tau)$ is a solution called the trivial solution. It is clear that $(X, r)$ is the trivial solution if and only if ${ }^{x} y=y$, and $x^{y}=x$, for all $x, y \in X$, or equivalently $\mathcal{L}_{x}=\mathrm{id}_{X}=\mathcal{R}_{x}$ for all $x \in X$. In this case $S(X, r)$ is the free abelian monoid, $G(X, r)$ is the free abelian group, $\mathcal{A}(k, X, r)$ the algebra of commutative polynomials in $X$, and $\mathcal{G}(X, r)=\left\{\operatorname{id}_{X}\right\}$ is the trivial group.

Remark 2.6. Suppose $(X, r)$ is a nondegenerate quadratic set. It is well known, see for example GIM08, that $(X, r)$ is a braided set (i.e. $r$ obeys the YBE) if and only if the following conditions hold

$$
\begin{gathered}
\text { 11: } \quad x(y z)={ }^{x} y\left(x^{y} z\right), \quad \mathbf{r 1}: \quad\left(x^{y}\right)^{z}=\left(x^{y} z\right)^{y^{z}}, \\
\left.\operatorname{lr3}: \quad\left({ }^{x} y\right)^{\left(x^{x^{y}}(z)\right)}={ }^{\left(x^{y} z\right.}\right)\left(y^{z}\right),
\end{gathered}
$$

for all $x, y, z \in X$.

Clearly, conditions 11 imply that, for each nondegenerate braided set $(X, r)$, the assignment $x \longrightarrow \mathcal{L}_{x}$ for $x \in X$ extends canonically to a group homomorphism

$$
\mathcal{L}: G(X, r) \longrightarrow \operatorname{Sym}(X),
$$

which defines the canonical left action of $G(X, r)$ on the set $X$. Analogously r1 gives the canonical right action of $G(X, r)$ on $X$. 
Definition 2.7. GIM08 Let $(X, r)$ be a nondegenerate braided set, $\mathcal{L}: G(X, r) \longrightarrow$ $\operatorname{Sym}(X)$ be the canonical group homomorphism defined via the left action. The image $\mathcal{L}(G(X, r))$ is denoted by $\mathcal{G}(X, r)$. We call it the (permutation) group of left action.

Remark 2.8. If $X$ is a finite set, then $\mathcal{G}=\mathcal{L}(S(X, r))$. Indeed, for a finite group, generating sets as group and as semigroup coincide; for any element $g$ of the group has finite order, say $m$, and so its inverse $g^{-1}$ can be expressed as a positive power $g^{m-1}$.

The following conditions were introduced and studied in [GI, GIM07, GIM08]:

Definition 2.9. GIM08 Let $(X, r)$ be a quadratic set.

(1) $(X, r)$ is called cyclic if the following conditions are satisfied

$$
\begin{aligned}
& \text { cl1 : } \quad y^{x} x={ }^{y} x \quad \text { for all } x, y \in X ; \quad \operatorname{cr} 1: \quad x^{x} y=x^{y}, \quad \text { for all } x, y \in X \text {; } \\
& \text { cl2: } \quad{ }^{x} y={ }^{y} x, \quad \text { for all } x, y \in X ; \quad \text { cr2 : } \quad x^{y^{x}}=x^{y} \quad \text { for all } x, y \in X \text {. }
\end{aligned}
$$

We refer to these conditions as cyclic conditions.

(2) Condition lri is defined as

$$
\text { lri: }\left({ }^{x} y\right)^{x}=y={ }^{x}\left(y^{x}\right) \text { for all } x, y \in X .
$$

In other words lri holds if and only if $(X, r)$ is nondegenerate and $\mathcal{R}_{x}=\mathcal{L}_{x}^{-1}$ and $\mathcal{L}_{x}=\mathcal{R}_{x}^{-1}$

2.1. Square-free solutions. In this paper the class of square-free solutions will be of special interest. We now introduce these.

In the case when $(X, r)$ is a square-free solution of finite order $|X|=n>2$, the algebras $\mathcal{A}(X, r)$ are binomial skew polynomial rings, see [GI, GI04, which provided new classes of Noetherian rings GI94, GI96-1, Gorentstein (Artin-Schelter regular) rings GI96-2, GI00, GI04 and so forth. Artin-Schelter regular rings were introduced in $\mathrm{AS}$, and are of particular interest. The algebras $\mathcal{A}(X, r)$ are similar in spirit to the quadratic algebras associated to linear solutions, particularly studied in $\mathrm{Ma}$, but have their own remarkable properties. The semigroups $S(X, r)$ were studied particularly in GIM08 with a systematic theory of 'exponentiation' from the set to the semigroup by means of the 'actions' $\mathcal{L}_{x}, \mathcal{R}_{x}$ (which in the process become a matched pair of semigroup actions) somewhat in analogy with the Lie theoretic exponentiation in $\mathrm{M} 90$.

We shall recall some basic facts and recent results needed in this paper.

The following result is extracted from GIM08, Theorem 2.34, where more equivalent conditions are pointed out. Note that in our considerations below (unless we indicate the contrary) the set $X$ is not necessarily of finite order.

Facts 2.10. GIM07. Suppose $(X, r)$ is nondegenerate, involutive and square-free quadratic set (not necessarily finite). Then the following conditions are equivalent:

(i) $(X, r)$ is a set-theoretic solution of the Yang-Baxter equation;

(ii) $(X, r)$ satisfies $\mathbf{1 1}$;

(iii) $(X, r)$ satisfies $\mathbf{r} \mathbf{1}$;

(iv) $(X, r)$ satisfies $\operatorname{lr} 3$. 
In this case $(X, r)$ is cyclic and satisfies lri.

Corollary 2.11. Every square-free solution $(X, r)$ satisfies lri, so it is uniquely determined by the left action $\mathcal{L}: X \times X \longrightarrow X$, more precisely,

$$
r(x, y)=\left(\mathcal{L}_{x}(y), \mathcal{L}_{y}^{-1}(x)\right) .
$$

Furthermore it is cyclic.

The following is straightforward and gives an alternative definition of square-free solutions.

Lemma 2.12. Let $X$ be a nonempty set and $\mathcal{L}$ be a map

$$
\mathcal{L}: X \longrightarrow \operatorname{Sym} X ; \quad x \mapsto \mathcal{L}_{x} \in \operatorname{Sym} X .
$$

Denote $\mathcal{L}_{x}(y)={ }^{x} y, \quad \mathcal{L}_{x}^{-1}(y)=y^{x}$ and define $r: X \times X \longrightarrow X \times X$ as $r(x, y)=$ $\left({ }^{x} y, x^{y}\right)$. Then $(X, r)$ is a square-free solution if and only if the following three conditions are satisfied for all $x, y, z \in X$ :

(i) ${ }^{x} x=x$

(ii) $\quad\left(y^{x} x\right)={ }^{y} x$

(iii) $\quad x(y)={ }^{x} y\left(x^{y} z\right)$

Remark 2.13. Note that in the hypothesis of Lemma 2.12 condition (ii) implies $(X, r)$ involutive.

Corollary 2.14. GIM08, In the hypothesis and notation of Lemma 2.12, $(X, r)$ is a square-free solution if and only if the following conditions are satisfied for all $x, y, z \in X$ :

$$
\begin{aligned}
& \text { (i) }{ }^{x} x=x \\
& \text { (ii) }{ }^{y} x\left({ }^{y} z\right)={ }^{x} y\left({ }^{x} z\right)
\end{aligned}
$$

Recall that a quadratic set $(X, r)$ which satisfies condition (ii) of Corollary 2.14 is called a cyclic set, see [Ru, GIM08].

Definition 2.15. The permutation group $H \subseteq \operatorname{Sym} X$ is called a YB permutation group, if it is isomorphic to $\mathcal{G}(X, r)$ for some square-free solution $(X, r)$.

Open questions 2.16. Let $X$ be a nonempty set.

(1) For which permutation groups $H \subseteq \operatorname{Sym} X$ is there a square-free solution $(X, r)$ with $\mathcal{G}(X, r)=H ?$

(2) Let $m$ be a positive integer. For which permutation groups $H \subseteq \operatorname{Sym} X$ is there a square-free solution $(X, r)$ with $\mathcal{G}(X, r)=H$ and $\operatorname{mpl}(X, r)=m$ (see Definition 2.25)?

The next result gives a translation of the first question which is not very easy to check.

Proposition 2.17. Let $H \subseteq \operatorname{Sym} X$ be a permutation group. Then $H$ is a $Y B$ permutation group for some square-free solution $(X, r)$ if and only if there exists a map

such that the following conditions hold:

$$
f: X \longrightarrow H ; \quad x \mapsto f_{x}
$$


(1) $f(X)$ is a generating set for $H$;

(2) $f_{x}(x)=x$;

(3) $f_{f_{y}(x)} \circ f_{y}=f_{f_{x}(y)} \circ f_{x}$

In this case the quadratic set $(X, r)$ with $r(x, y)=\left(f_{x}(y),\left(f_{y}\right)^{-1}(x)\right)$ is a square-free solution, and $H \simeq \mathcal{G}(X, r)$.

Proof. Corollary 2.14implies that the quadratic set $(X, r)$ is a square-free solution. Clearly, in this case $\mathcal{L}_{x}=f_{x} \in \operatorname{Sym} X$. Condition 3 (together with lri) implies

$$
f_{x} \circ f_{y}=f_{x y} \circ f_{x^{y}}
$$

so $\left(f_{x} \circ f_{y}\right)(a)=f_{x}\left(f_{y}(a)\right)$ and the map $\mathcal{L}_{x} \mapsto f_{x}$ extends to a group homomorphism $\varphi: \mathcal{G}(X, r) \longrightarrow H$. We have $f_{x_{i_{1}}} \circ \cdots f_{x_{i_{k}}}(y)={ }^{x_{i_{1}}}\left(\cdots\left({ }^{x_{i_{k}}} y\right) \cdots\right)$, so the kernel of $\varphi$ is trivial.

In Proposition 5.8 we describe the permutation groups $H \subseteq$ Sym $X$ which define square-free solutions $(X, r)$ with $\operatorname{mpl}(X, r)=2$, where $X$ is an arbitrary finite nonempty set.

Definition 2.18. Let $(X, r)$ be a braided set, $G=G(X, r), \mathcal{G}=\mathcal{G}(X, r)$. A subset $Y \subset X$ is said to be $r$-invariant if $r(Y \times Y) \subseteq Y \times Y$. Suppose $Y$ is an $r$-invariant subset of $(X, r)$. Then $r$ induces a solution $\left(Y, r_{\mid Y \times Y}\right)$. Denote $r_{\mid Y \times Y}=r_{\mid Y}$. We call $\left(Y, r_{\mid Y}\right)$ the restricted solution (on $Y$ ). We say that $Y \subset X$ is a (left) $G$ invariant subset of $X$, or equivalently a $\mathcal{G}$-invariant subset, if $Y$ is invariant under the left action of $G$. Clearly, $Y$ is (left) $G$-invariant if and only if

$$
\mathcal{L}_{a}(Y) \subseteq Y, \quad \forall a \in X .
$$

Right $G$-invariant subsets are defined analogously. In the case when $(X, r)$ is symmetric, and condition lri holds the subset $Y$ is left $G$-invariant if and only if it is right $G$-invariant. In this case we shall refer to it simply as a $\mathcal{G}$-invariant subset.

Clearly each $\mathcal{G}$-invariant subset $Y$ of $X$ is also an $r$-invariant subset, but, in general, an $r$-invariant subset may, or may not be $\mathcal{G}$-invariant. The following holds:

Lemma 2.19. Let $(X, r)$ be a symmetric set with lri, $\mathcal{G}=\mathcal{G}(X, r) . \quad Y \subset X$. Denote by $Z$ the complement of $Y$ in $X$. The following conditions are equivalent.

(1) $Y$ is $\mathcal{G}$-invariant;

(2) $Z$ is $\mathcal{G}$-invariant;

(3) $Y$ and $Z$ are $r$-invariant complementary subsets of $X$.

Moreover, in this case $(X, r)$ decomposes as a disjoint union of $r$-invariant subsets $X=Y \bigcup Z$.

Proof. Suppose $Y$ is $\mathcal{G}$-invariant. Clearly, $x \in Y$ if and only if the $\mathcal{G}$-orbit of $x$ is contained in $Y$.

It is easy to show now that $Z$ is also $\mathcal{G}$-invariant. Indeed assume $z \in Z, a \in X, t=$ ${ }^{a} z$. If we assume that $t$ is not in $Z$, this would imply $t \in Y$. By lri one has

$$
t={ }^{a} z \Longrightarrow t^{a}=\left({ }^{a} z\right)^{a}=z
$$


But $t \in Y$, and the $\mathcal{G}$-orbit of $t$ is contained in $Y$, so $z=t^{a} \in Y$, a contradiction. Thus $Z$ is $\mathcal{G}$-invariant. This proves $(1) \Longrightarrow(2)$.

The implication $(2) \Longrightarrow(1)$ is analogous. Clearly, then each of the conditions (1) and (2) implies that $Y$ and $Z$ are $r$-invariant.

Suppose now $(X, r)$ is decomposed into two $r$-invariant disjoint subsets $X=Y \bigcup Z$. We claim that (1) and (2) hold. It will be enough to verify (1). $Y$ is $r$-invariant, therefore

$$
{ }^{a} y \in Y \quad \forall a, y \in Y .
$$

It remains then to show that

$$
{ }^{z} y \in Y \quad \forall y \in Y, z \in Z .
$$

Assume the contrary, for some $y \in Y, z \in Z$, one has ${ }^{z} y \in Z$. This yield

$$
t={ }^{z} y \in Z \Longrightarrow y=\left({ }^{z} y\right)^{z}=t^{z} \in Z,
$$

since $Z$ s $r$-invariant. This contradicts $y \in Y$.

It is easy to show that $r_{Y}: Y \times Y \longrightarrow Y \times Y$ is surjective, and therefore bijective. Clearly, $\left(Y, r_{Y}\right)$ is involutive, hence $\left(Y, r_{Y}\right)$ is a symmetric set.

Note that $(X, r)$ is a square-free solution since in this case condition lri holds, see Corollary 2.11,

Remark 2.20. Suppose $(X, r)$ is a square-free solution. Then it satisfies lri (see Corollary 2.11) and therefore Lemma 2.19 is in force. Clearly, each $G$-orbit $X_{0}$ under the left action of $G$ on $X$ is $\mathcal{G}$-invariant and therefore it is an $r$-invariant subset. In the case when $G$ acts non-transitively on $X$ (in particular, this this holds when $X$ is finite), $(X, r)$ decomposes into a disjoint union $X=X_{0} \bigcup Z$, of its $r$-invariant subsets $X_{0}$, and $Z$, where $Z$ is the complement of $X_{0}$ in $X$.

Each finite involutive solution $(X, r)$ with lri can be represented geometrically by its graph of the left action $\Gamma(X, r)$. It is an oriented labeled multi-graph (although we refer to it as a graph). It was introduced in GI00 for square-free solutions, see also GIM07, Section 5, and GIM08. Here we recall the definition.

Definition 2.21. GIM07 Let $(X, r)$ be a finite symmetric set with lri. We define the graph $\Gamma=\Gamma(X, r)$ as follows. It is an oriented graph, which reflects the left action of $G(X, r)$ on $X$. The set of vertices of $\Gamma$ is $X$. There is a labeled arrow $x \stackrel{a}{\longrightarrow} y$ if $x, y, a \in X$ and ${ }^{a} x=y$. An edge $x \stackrel{a}{\longrightarrow} y$ with $x \neq y$ is called a nontrivial edge. We will often consider the simplified graph in which to avoid clutter we typically omit self-loops unless needed for clarity or contrast. Also for the same reason, we use the line type to indicate when the same type of element acts, rather than labeling every arrow. Clearly, $x \stackrel{a}{\longleftrightarrow} y$ indicates that ${ }^{a} x=y$ and ${ }^{a} y=x$. (One can make such graphs for arbitrary solutions but then it should be indicated which action is considered).

Note that two solutions are isomorphic if and only if their oriented graphs are isomorphic. Various properties of a solution $(X, r)$ are reflected in the properties of its graph $\Gamma(X, r)$, see for example the remark below.

Remark 2.22. Let $(Z, r)$ be a symmetric set with lri, $\Gamma=\Gamma(Z, r)$. 
(1) $(Z, r)$ is a square-free solution if and only if $\Gamma$ does not contain a nontrivial edge $x \stackrel{x}{\longrightarrow} y, x \neq y$; that is, the edge of type $x$ leaving $x$ is a loop.

(2) In this case, $(Z, r)$ is a trivial solution (or equivalently, $\operatorname{mpl}(Z, r)=1$ ) if and only if all the edges are loops.

(3) The $G$-orbits of $X$ are in 1-1 correspondence with the connected components of $\Gamma$.

(4) GIM07, Theorem 5.24 gives necessary and sufficient conditions for $\operatorname{mpl}(X, r)=$ 2 in terms of the properties of $\Gamma(X, r)$. It can be read off from GIM07, Theorem 5.22, Theorem 5.24 that, in this case, each nontrivial connected component $\Gamma_{i}$ of $\Gamma$ is a Cayley graph (see below).

Definition 2.23. If $G$ is a group and $S$ a subset of $G$, the Cayley graph Cay $(G, S)$ is the graph with vertex set $G$ and directed edges $(g, g s)$ for all $g \in G$ and $s \in S$.

Note that the group $G$ acts by left multiplication as automorphisms of the Cayley graph Cay $(G, S)$. The graph is loopless if and only if id $\notin S$ and connected if and only if $\langle S\rangle=G$.

Now any graph admitting a group $G$ of automorphisms acting regularly on the vertices is a Cayley graph for $G$. For the vertex set can be identified with $G$ (with action by left multiplication); and, if $S$ is the set of vertices $s$ for which (id, $s$ ) is an edge, then $(g, g s)$ is an edge for all $g \in G$ since $G$ acts by automorphisms. In particular, every transitive abelian group action is regular, so a graph with a transitive abelian group of automorphisms is a Cayley graph.

The notions of retraction of symmetric sets and multipermutation solutions were introduced in the general case in ESS, where $(X, r)$ is not necessarily finite, or square-free.

In GI, GIM07, GIM08, GIM0806 the multipermutation square-free solutions are studied; we recall some notions and results. Let $(X, r)$ be a nondegenerate symmetric set. An equivalence relation $\sim$ is defined on $X$ as

$$
x \sim y \quad \text { if and only if } \quad \mathcal{L}_{x}=\mathcal{L}_{y} .
$$

In this case we also have $\mathcal{R}_{x}=\mathcal{R}_{y}$,

We denote by $[x]$ the equivalence class of $x \in X,[X]=X / \sim$ is the set of equivalence classes.

Lemma 2.24. GIM08 Let $(X, r)$ be a nondegenerate symmetric set.

(1) The left and the right actions of $X$ onto itself naturally induce left and right actions on the retraction $[X]$, via

$$
{ }^{[\alpha]}[x]:=\left[{ }^{\alpha} x\right] \quad[\alpha]^{[x]}:=\left[\alpha^{x}\right], \text { for all } \alpha, x \in X .
$$

(2) The new actions define a canonical map $r_{[X]}:[X] \times[X] \longrightarrow[X] \times[X]$ where $r_{[X]}([x],[y])=\left({ }^{[x]}[y],[x]^{[y]}\right)$.

(3) $\left([X], r_{[X]}\right)$ is a nondegenerate symmetric set. Furthermore,

(4) $(X, r)$ cyclic $\Longrightarrow\left([X], r_{[X]}\right)$ cyclic.

(5) $(X, r)$ is lri $\Longrightarrow\left([X], r_{[X]}\right)$ is lri.

(6) $(X, r)$ square-free $\Longrightarrow\left([X], r_{[X]}\right)$ square-free. 
Definition 2.25. ESS The solution $\operatorname{Ret}(X, r)=([X],[r])$ is called the retraction of $(X, r)$. For all integers $m \geq 1, \operatorname{Ret}^{m}(X, r)$ is defined recursively as $\operatorname{Ret}^{m}(X, r)=$ $\operatorname{Ret}\left(\operatorname{Ret}^{m-1}(X, r)\right)$.

$(X, r)$ is a multipermutation solution of level $m$, if $m$ is the minimal number (if any), such that $\operatorname{Ret}^{m}(X, r)$ is the trivial solution on a set of one element. In this case we write $\operatorname{mpl}(X, r)=m$.

By definition $(X, r)$ is a multipermutation solution of level 0 if and only if $X$ is a one element set.

The following conjecture was made by the first author in 2004 .

Conjecture 2.26. GI

(1) Every finite square-free solution $(X, r)$ is retractable.

(2) Every finite square-free solution $(X, r)$ of finite order $n$ is multipermutation solution, with $\operatorname{mpl}(X, r)<n$.

A more recent conjecture states

Conjecture 2.27. GI08 Suppose $(X, r)$ is a nondegenerate square-free multipermutation solution of finite order $n$. Then $\operatorname{mpl}(X)<\log _{2} n$.

Evidence for this conjecture will be given later in the paper.

\section{Homomorphisms, AUtomorphisms, STRONG TWISTED UNIONS}

In this section, we recall the definitions and basic properties of homomorphisms and automorphisms of solutions, see and give a general construction, the strong twisted union of solutions, see GIM07, GIM08.

Definition 3.1. GIM07 Let $\left(X, r_{X}\right)$ and $\left(Y, r_{Y}\right)$ be arbitrary solutions (braided sets). A map $\varphi: X \longrightarrow Y$ is a homomorphism of solutions, if it satisfies the equality

$$
(\varphi \times \varphi) \circ r_{X}=r_{Y} \circ(\varphi \times \varphi) \text {. }
$$

A bijective homomorphism of solutions is called (as usual) an isomorphism. An isomorphism of the solution $(X, r)$ onto itself is an $r$-automorphism.

We denote by $\operatorname{Hom}\left(\left(X, r_{X}\right),\left(Y, r_{Y}\right)\right)$ the set of all homomorphisms of solutions $\varphi: X \longrightarrow Y$. The group of $r$-automorphisms of $(X, r)$ will be denoted by $\operatorname{Aut}(X, r)$.

Clearly, $\operatorname{Aut}(X, r)$ is a subgroup of $\operatorname{Sym}(X)$.

Remark 3.2. GIM07 Let $\left(X, r_{X}\right),\left(Y, r_{Y}\right)$ be finite square-free solutions Every homomorphism of solutions $\varphi:\left(X, r_{X}\right) \longrightarrow\left(Y, r_{Y}\right)$ induces canonically a homomorphism of their graphs:

$$
\varphi_{\Gamma}: \Gamma\left(X, r_{X}\right) \longrightarrow \Gamma\left(Y, r_{Y}\right) .
$$

Furthermore there is a one-to one correspondence between $\operatorname{Aut}(X, r)$ and $\operatorname{Aut}(\Gamma(X, r))$, the group of automorphisms of the multigraph $\Gamma(X, r)$.

Lemma 3.3. GIM07 Let $\left(X, r_{X}\right)$ and $\left(Y, r_{Y}\right)$ be braided sets. 
(1) $A \operatorname{map} \varphi: X \longrightarrow Y$ is a homomorphism of solutions if and only if $\varphi \circ \mathcal{L}_{x}=\mathcal{L}_{\varphi(x)} \circ \varphi$ and $\varphi \circ \mathcal{R}_{x}=\mathcal{R}_{\varphi(x)} \circ \varphi$ for all $x \in X$.

(2) If both $\left(X, r_{X}\right)$ and $\left(Y, r_{Y}\right)$ satisfy lri, then $\varphi$ is a homomorphism of solutions if and only if

$$
\varphi \circ \mathcal{L}_{x}=\mathcal{L}_{\varphi(x)} \circ \varphi, \text { for all } x \in X .
$$

(3) If $(X, r)$ obeys lri, (in particular, if $(X, r)$ is a square-free solution) then $\sigma \in \operatorname{Sym}(X)$ is an automorphism of $\left(X, r_{X}\right)$ if and only if

$$
\sigma \circ \mathcal{L}_{x} \circ \sigma^{-1}=\mathcal{L}_{\sigma(x)}, \text { for all } x \in X .
$$

For example, if $(X, r)$ is the trivial solution then, clearly, $\operatorname{Aut}(X, r)=\operatorname{Sym}(X)$. This is because each $\mathcal{L}_{x}=\operatorname{id}_{X}$. More generally, (3.1) implies:

Corollary 3.4. GIM07] The group $\operatorname{Aut}(X, r)$ is a subgroup of $\operatorname{Nor}_{\operatorname{Sym}(X)} \mathcal{G}(X, r)$, the normalizer of $\mathcal{G}(X, r)$ in $\operatorname{Sym}(X)$.

Corollary 3.5. Suppose $(X, r)$ is a braided set with lri. Let $Y$, be an $r$ - invariant subset, $\left(Y, r_{\mid Y}\right)$ be the restricted solution. Let $x \in X$. Then $\mathcal{L}_{x} \in \operatorname{Aut}\left(Y, r_{\mid Y}\right)$ if and only if

$$
\left(\mathcal{L}_{\alpha}\right)_{\mid Y}=\left(\mathcal{L}_{x}\right)_{\mid Y} \quad \forall \alpha \in Y
$$

Corollary 3.6. Suppose $(X, r)$ is a square-free solution. Then the following conditions hold.

(1) For each $r$ - invariant subset $Y$, and each $x \in X$, one has $\mathcal{L}_{x} \in \operatorname{Aut}\left(Y, r_{\mid Y}\right)$ if and only if (3.2) holds.

(2) The intersection $\mathcal{G}_{0}=\operatorname{Aut}(X, r) \bigcap \mathcal{G}$ is an abelian subgroup of $\mathcal{G}$.

Proof. We know that each square-free solution satisfies lri, hence, Corollary 3.5 gives (1). By Corollary 2.14 (ii) the following equality holds:

$$
\mathcal{L}_{x}{ }_{y} \circ \mathcal{L}_{x}=\mathcal{L}_{y}{ }_{x} \circ \mathcal{L}_{y} \quad \forall x, y \in X
$$

Assume now that $\mathcal{L}_{x}, \mathcal{L}_{y} \in \operatorname{Aut}(X, r)$. Then (1) implies

$$
\begin{array}{lll}
\mathcal{L}_{y_{x}}=\mathcal{L}_{x} & : \quad \text { by } & \mathcal{L}_{x} \in \operatorname{Aut}(X, r) \\
\mathcal{L}^{x} y=\mathcal{L}_{y} & : \quad \text { by } \quad \mathcal{L}_{y} \in \operatorname{Aut}(X, r)
\end{array}
$$

This together with (3.3) yield

$$
\mathcal{L}_{x} \circ \mathcal{L}_{y}=\mathcal{L}_{y} \circ \mathcal{L}_{x}
$$

We shall now discuss a special class of extensions of solutions called strong twisted unions of solutions

Definition 3.7. GIM07, GIM08, Let $(X, r)$ be an involutive quadratic set, suppose $X$ is a disjoint union $X=X_{1} \bigcup X_{2}$ of $r$-invariant subsets. Suppose the restricted sets $\left(X_{1}, r_{1}\right),\left(X_{2}, r_{2}\right)$ are symmetric sets $\left(r_{i}=r_{\mid X_{i}}, i=1,2\right)$. The quadratic set $(X, r)$ is a strong twisted union of $\left(X_{1}, r_{1}\right)$ and $\left(X_{2}, r_{2}\right)$ if 
(1) The assignment $\alpha \longrightarrow \mathcal{L}_{\alpha \mid X_{1}}, \alpha \in X_{2}$, extends to a left action of the associated group $G\left(X_{2}, r_{2}\right)$ (and the associated monoid $S\left(X_{2}, r_{2}\right)$ ) on $X_{1}$, and the assignment $x \longrightarrow \mathcal{L}_{x \mid X_{2}}, x \in X_{1}$ extends to a right action of the associated group of $G\left(X_{1}, r_{1}\right)$ (and the associated monoid $S\left(X_{1}, r_{1}\right)$ ) on $X_{2}$;

(2) The actions satisfy

$$
\text { stu : } \quad \alpha^{y} x={ }^{\alpha} x ; \quad \alpha^{\beta} x=\alpha^{x}, \quad \text { for all } \quad x, y \in X, \alpha, \beta \in Y .
$$

Note first that condition stu is equivalent to the following condition:

$$
\text { stu1 : } \quad{ }^{y} \alpha={ }^{\alpha} x ; \quad \alpha^{x^{\beta}}=\alpha^{x}, \quad \text { for all } \quad x, y \in X_{1}, \alpha, \beta \in X_{2} .
$$

We shall refer to it also as stu condition.

Secondly, note that by Definition 3.7 a strong twisted union of symmetric sets is not necessarily a solution (a symmetric set). We shall use notation

$$
(X, r)=X_{1} \curvearrowleft X_{2}
$$

to denote that $(X, r)$ is a symmetric set which is a strong twisted union of its $r$-invariant subsets $X_{1}$ and $X_{2}$.

The strong twisted union $(X, r)$ of $\left(X_{1}, r_{1}\right)$ and $\left(X_{2}, r_{2}\right)$ is nontrivial if at least one of the actions in (11) is nontrivial. In the case when both actions (1) are trivial we write $\left.(X, r)=X_{1} \natural_{0} X_{2}\right)$. In this case one has $r(x, \alpha)=(\alpha, x), r(\alpha, x)=(x, \alpha)$ for all $x \in X_{1}, \alpha \in X_{2}$.

In GIM07, GIM08 and GIM0806 appear strong twisted unions of $m$ disjoint symmetric sets, where $m$ is arbitrary integer, $m \geq 2$. Although a formal definition was not given, the notion of strong twisted union there is clear from the context. Here we give a formal definition.

Definition 3.8. Let $(X, r)$ be a symmetric set, which is a disjoint union of $\mathcal{G}$ invariant subsets $X_{1}, \cdots, X_{m}$, where $m \geq 2$. For each pair $i \neq j, 1 \leq i, j \leq m$, denote by $X_{i j}$ the $r$-invariant subset $X_{i j}=X_{i} \bigcup X_{j}$. We say that $(X, r)$ is a $a$ strong twisted union of $\left(X_{i}, r_{i}\right), 1 \leq i \leq m$ and write

$$
X=X_{1} \natural X_{2} \natural \cdots \curvearrowleft X_{m}
$$

if

$$
X_{i j}=X_{i} \curvearrowleft X_{j} \quad, \forall i \neq j, 1 \leq i, j \leq m .
$$

Here as usual, $\left(X_{i}, r_{i}\right), 1 \leq i, j \leq m$ denotes the symmetric set with $r_{i^{-}}$the restriction of $r$ on $X_{i} \times X_{i}$.

Example 3.9. Let $\left(X_{1}, r_{1}\right), \cdots,\left(X_{s}, r_{s}\right)$ be pairwise disjoint square-free solutions. Let $X=\bigcup_{1 \leq i \leq s} X_{i}$. Let $r: X \times X \longrightarrow X \times X$ be the extension of $r_{i}, 1 \leq i \leq s$ satisfying $r(x, \alpha)=(\alpha, x)$ whenever $x \in X_{i}, \alpha \in X_{j}$, where $1 \leq i, j \leq s, i \neq j$. Then clearly $(X, r)$ is a square-free solution and $(X, r)=X_{i} \natural_{0} X_{2} \natural_{0} \cdots \natural_{0} X_{s}$.

Note that when $(X, r)$ is a strong twisted union $X=X_{1} \natural X_{2} \natural \cdots \natural X_{m}$ of $m$ $\mathcal{G}$-invariant subsets, $m>2$, each set $X_{i}$, and its complement $Z_{i}$ are $\mathcal{G}$-invariant. (Clearly, $Z_{i}=\bigcup_{1 \leq j \leq m, j \neq i} X_{j}$ ). However, the union $X_{i} \bigcup Z_{i}$ may not be a strong twisted union of solutions. 
Corollary 3.10. Let $(X, r)$ be a square-free solution. Suppose $X_{1}, X_{2}, \cdots, X_{m}$ are disjoint $\mathcal{G}$-invariant subsets of $X$.

Then $(X, r)$ is a strong twisted union $X=X_{1} \curvearrowleft X_{2} \sharp \cdots \curvearrowleft X_{m}$ if and only if for each pair $i \neq j, 1 \leq i, j \leq m$ and each $x \in X_{i}$ one has

$$
\left(\mathcal{L}_{x}\right)_{\mid X_{j}} \in \operatorname{Aut}\left(X_{j}, r_{j}\right) .
$$

Definition 3.11. Suppose $(Z, r)$ is an extension (nondegenerate, involutive) of the square-free disjoint solutions $\left(X, r_{X}\right)$ and $\left(Y, r_{Y}\right)$. Consider the maps

$$
f=f(X, Y): Z \times Z \longrightarrow Z \times Z \quad g=g(Y, X): Z \times Z \longrightarrow Z \times Z
$$

defined for all $x, y \in X, \alpha, \beta \in Y$ as

$$
\begin{array}{llll}
f(\alpha, x)=\left({ }^{\alpha} x, \alpha\right) & f(x, \alpha)=\left(\alpha, x^{\alpha}\right) & f_{\mid X \times X}=r_{X}, & f_{\mid Y \times Y}=\tau_{Y} \\
g(x, \alpha)=\left({ }^{x} \alpha, x\right) & g(\alpha, x)=\left(x, \alpha^{x}\right) & g_{\mid X \times X}=\tau_{X}, & g_{\mid Y \times Y}=\tau_{Y}
\end{array}
$$

(Here $\tau_{X}, \tau_{Y}$ are the corresponding flips, and the left and the right actions ${ }^{\alpha} \bullet, \cdots, \bullet$ are the canonical actions defined via $r$ ).

We call $f$ and $g$, respectively, the associated $X$ - and $Y$-split maps of $r$.

Proposition 3.12. Suppose the quadratic set $(Z, r)$ is an extension (nondegenerate, involutove) of the square-free disjoint solutions $\left(X, r_{X}\right)$ and $\left(Y, r_{Y}\right)$, let $f, g$ be respectively the associated $X-$, respectively, $Y$ - split maps of $r$. Then the following condition holds.

(i) $f$ and $g$ are involutive maps.

(ii) There is an equality of maps $r=f \circ \tau \circ g$.

Suppose furthermore that $(Z, r)$ is a square-free solution.

$(Z, f) \quad$ is a square-free solution $\Longleftrightarrow \quad \alpha^{y} x={ }^{\alpha} x \quad \forall x, y \in X, \alpha \in Y$.

(iv)

In this case $G\left(Y, r_{Y}\right)$ acts as automorphisms on $\left(X, r_{X}\right)$.

$(Z, g) \quad$ is a square-free solution $\Longleftrightarrow{ }^{x^{\beta}} \alpha={ }^{x} \alpha \quad \forall x \in X, \alpha, \beta \in Y$

In this case $G\left(X, r_{X}\right)$ acts as automorphisms on $\left(Y, r_{Y}\right)$.

(v)

$(Z, f)$ and $(Z, g)$ are square-free solutions $\Longleftrightarrow \quad(Z, r)=X \sharp Y$.

Proof. Let $x, y \in X$ and $\alpha, \beta \in Y$. We look at the diagrams (3.6). The left handside diagram contains all elements of the orbit of monomial $\alpha x y \in X^{3}$, under the action of the group $\mathcal{D}(f)={ }_{g r}\left\langle f^{12}, f^{23}\right\rangle$. Analogously the right hand-side diagram contains the elements of the orbit of $x \alpha \beta \in X^{3}$ under the action of $\mathcal{D}(g)=$ 
${ }_{g r}\left\langle g^{12}, f g^{23}\right\rangle$.

(3.6)
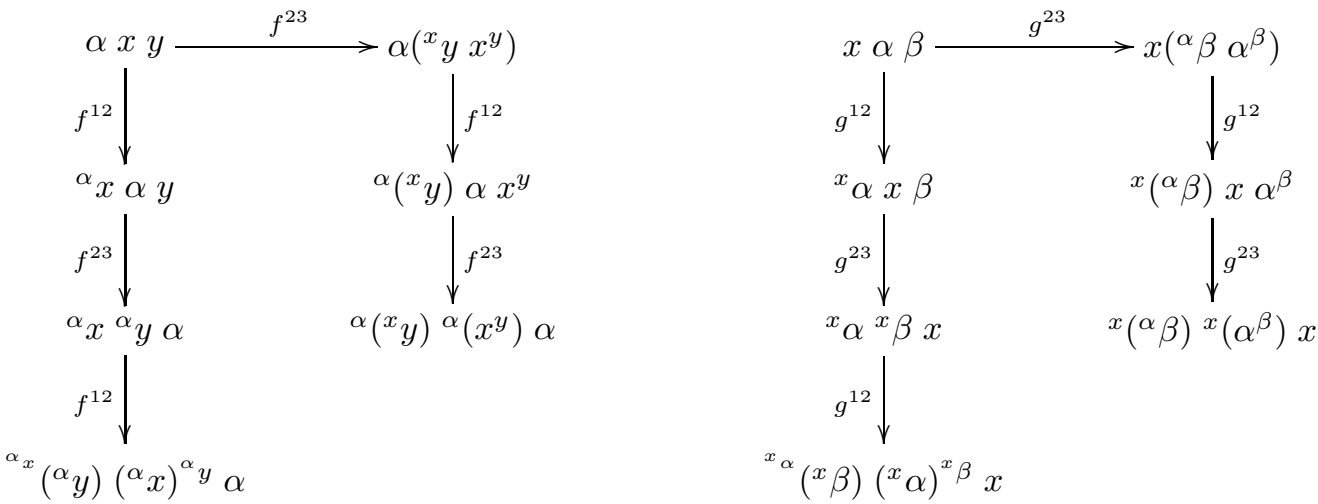

Suppose $f$ is a solution, then from the left hand-side diagram we obtain an equality of words in the free monoid $\langle X\rangle$ :

$$
{ }^{\alpha_{x}}\left({ }^{\alpha} y\right)\left({ }^{\alpha} x\right)^{\alpha} y={ }^{\alpha}\left({ }^{x} y\right){ }^{\alpha}\left(x^{y}\right) \alpha,
$$

and therefore

$$
{ }^{\alpha_{x}}\left({ }^{\alpha} y\right)={ }^{\alpha}\left({ }^{x} y\right)
$$

By assumption, $r$ is a solution, so $\mathbf{1 1}$ gives

$$
{ }^{\alpha}\left({ }^{x} y\right)={ }^{\alpha x}\left(\alpha^{x} y\right),
$$

Now (3.7) and (3.8) imply

$$
{ }^{\alpha}\left({ }^{\alpha} y\right)={ }^{\alpha}\left({ }^{\alpha^{x}} y\right),
$$

which by the non degeneracy of $(Z, r)$ implies

$$
{ }^{\alpha} y={ }^{\alpha^{x}} y,
$$

and this is valid for all $x, y \in X, \alpha \in Y$.

Assume now that $(Z, r)$ is a solution. Then direct computation to shows that (3.9) implies that $(Z, f)$ satisfies condition $\mathbf{1 1}$, and therefore is a square-free solution.

Analogous argument shows that under the assumption that $(Z, r)$ is a solution, $(Z, g)$ is a square-free solution if and only if

$$
{ }^{x} \alpha={ }^{x^{\beta}} \alpha \quad \forall x \in X, \alpha, \beta \in Y
$$

Clearly, both $(Z, f)$ and $(Z, g)$ are square free solutions if and only if both (3.9) and (3.10) hold, which by Corollary is equivalent to

$$
(Z, r)=X \natural Y .
$$




\section{Decomposition of solutions.}

In this section we study various decompositions of square-free solutions $(X, r)$ into disjoint unions of a finite number of $r$-invariant subsets and the corresponding factorisation of $S(X, r), G(X, r)$, and $\mathcal{G}(X, r)$. We use essentially the matched pairs approach to solutions (in the most general setting) developed in GIM08 . We first recall the necessary notions and results from [GIM08.

4.1. The matched pairs approach to set-theoretic YBE. The notion of a matched pair of groups in relation to group factorisation has a classical origin. By now there have been various works on matched pairs in different contexts and we refer to the text in GIM08 and references therein. In particular, this notion was used by Lu, Yan and Zhu to study the set-theoretic solution of YBE and the associated 'braided group', see [LYZ and the excellent review [Ta]. The notion of a matched pair of monoids, is developed in GIM08 with additional refinements that disappear in the group case.

We now recall some notions and results from GIM08.

Definition 4.1. $(S, T)$ is a matched pair of monoids if $T$ acts from the left on $S$ by ( ) $\bullet$ and $S$ acts on $T$ from the right by $\bullet$ ( ) and these two actions obey

$$
\begin{array}{cccc}
\text { ML0 : } & { }^{a} 1=1, \quad{ }^{1} u=u ; & \text { MR0 : } & 1^{u}=1, \quad a^{1}=a \\
\text { ML1 : } & \left({ }^{a b}\right) u={ }^{a}\left({ }^{b} u\right), & \text { MR1 : } & a^{(u v)}=\left(a^{u}\right)^{v} \\
\text { ML2 : } & { }^{a}(u . v)=\left({ }^{a} u\right)\left({ }^{a^{u}} v\right), & \text { MR2 : } & (a . b)^{u}=\left(a^{b} u\right)\left(b^{u}\right),
\end{array}
$$

for all $a, b \in T, u, v \in S$.

Proposition 4.2. GIM08 A matched pair $(S, T)$ of monoids implies the existence of a monoid $S \bowtie T$ (called the double cross product) built on $S \times T$ with product and unit

$$
(u, a)(v, b)=\left(u .^{a} v, a^{v} \cdot b\right), \quad 1=(1,1), \quad \forall u, v \in S, a, b \in T
$$

and containing $S, T$ as submonoids. Conversely, suppose that there exists a monoid $R$ factorising into monoids $S, T$ in the sense that (i) $S, T \subseteq R$ are submonoids and (ii) the restriction of the product of $R$ to a map $\mu: S \times T \rightarrow R$ is bijective. Then $(S, T)$ are a matched pair and $R \cong S \bowtie T$ by this identification $\mu$.

Definition 4.3. A strong monoid factorisation is a factorisation in submonoids $S, T$ as above such that $R$ also factorises into $T, S$. We say that a matched pair is strong if it corresponds to a strong factorisation.

Definition 4.4. A braided monoid is a monoid $S$ forming part of a matched pair $(S, S)$ such that

(1) The equality

$$
u v=\left({ }^{u} v\right)\left(u^{v}\right) \text { holds in } S, \forall u, v \in S .
$$

(2) The associated map $r_{S}$

$$
r_{S}: S \times S \rightarrow S \times S \quad \text { defined by } \quad r_{S}(u, v)=\left({ }^{u} v, u^{v}\right)
$$

is bijective and obeys the YBE. A braided monoid is denoted by $\left(S, r_{S}\right)$.

(3) The braided monoid $\left(S, r_{S}\right)$ is called a strong braided monoid if $(S, S)$ is a strong matched pair. 
Remark 4.5. Matched pairs of groups and braided groups are defined analogously. Note that if the group $G$ forms a matched pair $(G, G)$ such that $u v=\left({ }^{u} v\right)\left(u^{v}\right)$ holds for all $u, v \in G$ then the associated map $r_{G}: G \times G \rightarrow G \times G$ with $r_{G}(u, v):=\left({ }^{u} v, u^{v}\right)$ is a solution of YBE so $\left(G, r_{G}\right)$ is a braided group.

The following facts can be extracted from [GIM08 Theorems 3.6 and 3.14.

Facts 4.6. Let $(X, r)$ be a braided set and $S=S(X, r)$ the associated monoid. Then

(1) The left and the right actions

$$
\text { ( ) }: X \times X \longrightarrow X \text {, and } \bullet \text { ( ) }: X \times X \longrightarrow X
$$

defined via $r$ can be extended in a unique way to a left and a right action

$$
\text { ( ) } \bullet: S \times S \longrightarrow S \text {, and } \bullet \text { ( ) }: S \times S \longrightarrow S \text {. }
$$

which make $\left(S, r_{S}\right)$ a strong (graded) braided monoid. (In particular $\left(S, r_{S}\right)$ is a set-theoretic solution of $Y B E$ ).

(2) $\left(S, r_{S}\right)$ is nondegenerate if and only if $(X, r)$ is nondegenerate.

(3) $\left(S, r_{S}\right)$ is involutive if and only if $(X, r)$ is involutive.

The following is an interpretation of GIM08, Remark 4.3 in our settings.

Remark 4.7. Suppose the square-free solution $(Z, r)$ decomposes into a disjoint union of its $r$-invariant (nonempty) subsets $X$ and $Y$. By definition $(Z, r)$ is nondegenerate, thus the equalities $r(x, y)=\left({ }^{x} y, x^{y}\right), r(y, x)=\left({ }^{y} x, y^{x}\right)$, and the nondegeneracy of $r, r_{X}, r_{Y}$ imply that

$$
{ }^{y} x, x^{y} \in X \text {, and }{ }^{x} y, y^{x} \in Y \text {, for all } x \in X, y \in Y .
$$

Therefore, $r$ induces bijective maps

$$
\rho: Y \times X \longrightarrow X \times Y \text {, and } \sigma: X \times Y \longrightarrow Y \times X,
$$

and left and right "actions"

$$
\begin{aligned}
& \text { ( ) }: Y \times X \longrightarrow X, \bullet \text { ( ) }: Y \times X \longrightarrow Y \text {, projected from } \rho \\
& \triangleright: X \times Y \longrightarrow Y, \quad \triangleleft: X \times Y \longrightarrow X, \text { projected from } \sigma .
\end{aligned}
$$

In the general setting of GIM08, extensions satisfying (4.2) are called regular extension.

4.2. Decompositions of square-free solutions, and factorisation of $S, G$ and $\mathcal{G}$. From now on we keep the conventions and the usual notation of this paper$(X, r)$ will denotes a square-free solution, not necessarily finite (unless we indicate the contrary). $S=S(X, r), G=G(X, r), \mathcal{G}=\mathcal{G}(X, r)$ denote respectively the YB-monoid, YB-group and the YB permutation group associated with $(X, r) . \mathcal{L}$ : $G(X, r) \longrightarrow \operatorname{Sym}(X)$ is the canonical group homomorphism defined via the left action, see Remark 2.6, and by definition $\mathcal{G}=\mathcal{L}(G(X, r))$. One has $\mathcal{G}=\mathcal{L}(S(X, r))$ whenever $X$ is a finite set.

It is known that when $(X, r)$ is a finite square-free solution the group $G$, or equivalently $\mathcal{G}$, acts intransitively on $X$. This follows from the decomposition theorem of Rump, $[\mathrm{Ru}$. Most of the statements in this and the later sections do not need necessarily the assumption that $X$ is of finite order. 
Remark 4.8. The group $\mathcal{G}$ acts intransitively on $X$ whenever $(X, r)$ is a square-free solution with finite multipermutation level $m \geq 1$ (where $X$ is a set of arbitrary cardinality). In this case the number of orbits $t$ equals at least the cardinality of the $(m-1)$ th retract, $\left|\operatorname{Ret}^{m-1}(X, r)\right|$, see Corollary 7.9 .

When the set $X$ is infinite we shall often impose the restriction that the number $t$ of $\mathcal{G}$-orbits is finite, this will be clearly indicated.

Notation 4.9. $\mathcal{O}_{\mathcal{G}}(x)$ will denote the $\mathcal{G}$-orbit of $x, x \in X$.

In all cases when $X$ has finite number of $\mathcal{G}$-orbits we shall denote these by $X_{1}, \cdots, X_{t}$.

Clearly, the orbits are $r$-invariant subsets of $X$, and each $\left(X_{i}, r_{i}\right), 1 \leq i \leq t$, where $r_{i}$ is the restriction $r_{i}=r_{\mid X_{i}}$, is also a square-free solution. For each $\alpha \in X_{j}$ there is an equality of sets

$$
X_{j}=\left\{{ }^{u} \alpha \mid u \in \mathcal{G}\right\} .
$$

Notation 4.10. Let $(X, r)$ be a square-free solution, $Y \subset X$ an arbitrary subset. We shall use the following notation.

$$
\begin{aligned}
& S(Y):=\text { the submonoid of } S(X, r) \text { generated by } Y ; \\
& G(Y):=\text { the subgroup of } G(X, r) \text { generated by } Y ;
\end{aligned}
$$

$\mathcal{G}(Y):=\mathcal{L}(G(Y))$. This is a subgroup of $\mathcal{G}(X, r)$ generated by the permutations $\mathcal{L}_{y} \in \mathcal{G}(X, r)$, where $y \in Y:$

$$
\mathcal{G}(Y)=g r\left\langle\mathcal{L}_{y} \in \mathcal{G}(X, r) \mid y \in Y\right\rangle
$$

Remark 4.11. Let $Y \subset X$, an arbitrary subset. Then $\mathcal{G}(Y)=1$ if and only if $Y \subset \operatorname{ker} \mathcal{L}$.

Remark 4.12. Suppose $Y$ is $\mathcal{G}$-invariant. Then $\left(Y, r_{Y}\right)$ is a square-free solution, (as usual $r_{Y}$ denotes the restriction $r_{Y \times Y}$ of $r$ ). Then $S(Y) \simeq S\left(Y, r_{Y}\right), G(Y) \simeq$ $G\left(Y, r_{Y}\right)$, (see Theorem 4.16). Note that in general, $\mathcal{G}(Y)$ is different from the permutation group $\mathcal{G}\left(Y, r_{\mid Y}\right) \leq \operatorname{Sym}(Y)$. Furthermore, if $(X, r)$ is a finite solution and $Y$ is an $r$-invariant subset of $X$, then the group $\mathcal{G}(Y)$ is the image of $S(Y)$ under the map $\mathcal{L}: G(X, r) \longrightarrow \operatorname{Sym}(X)$.

Proposition 4.13. Let $(X, r)$ be a square-free solution, $S=S(X, r), G=G(X, r)$. Suppose $Y$ is a $G$-invariant subset of $X$. Then

(i) ${ }^{a} u \in S(Y), \quad u^{a} \in S(Y) \quad \forall u \in S(Y), a \in S$;

(ii) $\quad{ }^{a} u \in G(Y), \quad u^{a} \in G(Y) \quad \forall u \in G(Y), a \in G$.

Under the hypothesis of the proposition we prove first the following key lemma.

Lemma 4.14. With the assumptions and notation of Proposition 4.13, the following are equalities in $G$

$$
{ }^{a}\left(y^{-1}\right)=\left({ }^{a} y\right)^{-1} ; \quad\left(y^{-1}\right)^{a}=\left(y^{a}\right)^{-1} \quad \forall a \in G, \quad y \in X .
$$

Proof. Note that each element $a \in G$ can be presented as a monomial

$$
a=\zeta_{1} \zeta_{2} \cdots \zeta_{n}, \quad \zeta_{i} \in X \bigcup X^{-1} .
$$


We shall consider a reduced form of $a$, that is a presentation 4.6 with minimal length $n$. We shall use induction on the length $n$ of the reduced form of $a$.

Step 1. $a \in X \bigcup X^{-1}$. Two cases are possible: (i) $a \in X$. By the cyclic condition we have ${ }^{y} a={ }^{a} y$. This implies

$$
\left({ }^{y} y\right) \cdot\left({ }^{a} y\right)^{-1}=1
$$

Recall that $(G, G)$ is a matched pair of groups, thus ${ }^{a} 1=1$ for all $a \in G$. Consider the equalities

$$
\begin{aligned}
& 1={ }^{y} a 1={ }^{y} a\left(y \cdot y^{-1}\right) \quad \text { : by MLO } \\
& \left.=\left[{ }^{y} a y\right] \cdot\left[{ }^{y} a\right)^{y}\left(y^{-1}\right)\right] \quad: \text { by ML2 } \\
& ={ }^{y} a y \cdot\left({ }^{a}\left(y^{-1}\right) \quad: \text { since }\left({ }^{y} a\right)^{y}=a\right. \text { by lri. }
\end{aligned}
$$

Hence

$$
1=\left({ }^{y} a\right) \cdot\left({ }^{a} y\right)^{-1}={ }^{y} a y \cdot\left({ }^{a}\left(y^{-1}\right),\right.
$$

which is an equality in the group $G$, therefore the left hand side of (4.5) holds. For the right hand side one uses analogous argument. By the (right) cyclic condition one has $y^{a^{y}}=y^{a}$, hence

$$
\left(y^{a}\right)^{-1}\left(y^{a^{y}}\right)=1
$$

This time we act on the right-hand side:

$$
\begin{aligned}
1=1^{a^{y}} & =\left(y^{-1} y\right)^{a^{y}} & & \text { : by MR0 } \\
& =\left[\left(y^{-1}\right)^{y}\left(a^{y}\right)\right]\left[y^{\left(a^{y}\right)}\right] & & : \text { by MR2 } \\
& =\left[\left(y^{-1}\right)^{a}\right]\left[y^{\left(a^{y}\right)}\right] & & \text { by lri. }
\end{aligned}
$$

So $\left(y^{-1}\right)^{a}\left(y^{\left(a^{y}\right)}\right)=\left(y^{a}\right)^{-1}\left(y^{a^{y}}\right)$ holds in $G$ and therefore $\left(y^{-1}\right)^{a}=\left(y^{a}\right)^{-1}$. This verifies RHS of (4.5). (ii) $a \in X^{-1}$, or equivalently $a=\zeta^{-1}$, where $\zeta \in X$. Recall that there is an equality

$$
\zeta^{-1} y=y^{\zeta} \quad \forall \zeta, y \in X
$$

Consider now the equalities:

$$
\begin{aligned}
& 1={ }^{y}\left(\zeta^{-1}\right)\left[y y^{-1}\right] \quad \text { : by MLO } \\
& \left.=\left({ }^{y} \zeta\right)^{-1}\right)\left[y y^{-1}\right] \quad \text { : by case } \mathbf{i} \\
& =\left[\left({ }^{\left.\left({ }^{y} \zeta\right)^{-1}\right)} y\right] \cdot\left[\left({ }^{y} \zeta\right)^{-1}\right)^{y} y^{-1}\right] \quad \text { : by ML2 } \\
& =\left[y^{(y \zeta)}\right] \cdot\left[\left({ }^{y} \zeta\right)^{y}\right)^{-1}\left(y^{-1}\right) \quad \text { : by case } \mathbf{i} \\
& =\left[y^{\zeta}\right] \cdot\left[\zeta^{-1}\right)\left(y^{-1}\right) \quad \text { : by the cyclic condition and lri } \\
& =\left[\zeta^{-1} y\right]\left[\left(^{-1}\right)\left(y^{-1}\right)\right. \text {. }
\end{aligned}
$$

So the equality

$$
\left[\zeta^{-1} y\right]\left[\zeta^{\left(\zeta^{-1}\right)} y^{-1}\right]=1
$$

implies

$$
\left[\zeta^{-1} y\right]^{-1}={ }^{\left(\zeta^{-1}\right)} y^{-1}
$$

and therefore

$$
\left({ }^{a} y\right)^{-1}={ }^{a}\left(y^{-1}\right) .
$$

This proves the LHS of (4.5). Analogous argument verifies its RHS. 
Step 2. Assume (4.5) hold for each $y \in X$ and each $a \in G$ with reduced form of minimal length $n$. Suppose $a \in G$ has minimal length $n+1$. Then $a=\zeta b$, where $\zeta \in X \bigcup X^{-1}$, and $b \in G$ has length $n$. Then

$$
\begin{aligned}
{ }^{a}\left(y^{-1}\right) & ={ }^{(\zeta b)}\left(y^{-1}\right) & & \\
& ={ }^{\zeta}\left({ }^{b}\left(y^{-1}\right)\right) & & : \text { by ML1 } \\
& ={ }^{\zeta}\left(\left({ }^{b} y\right)^{-1}\right) & & : \text { by the inductive assumption } \\
& =\left({ }^{\zeta}\left({ }^{b} y\right)\right)^{-1} & & : \text { by the inductive assumption } \\
& =\left({ }^{(\zeta b)} y\right)^{-1} & & : \text { by } \mathbf{M L 1} \\
& =\left({ }^{a} y\right)^{-1} & & : \text { by } a=\zeta b .
\end{aligned}
$$

This proves the LHS of (4.5), the remaining part is proven analogously. The lemma has been proved.

Proof of the proposition. We shall prove the implication

$$
u \in G(Y), a \in G \Longrightarrow{ }^{a} u \in G(Y) \quad a^{u} \in G(Y)
$$

This time we use induction on the length of $u$. Lemma 4.14 gives the base for the induction. Assume now (4.7) holds for all $u \in G(Y)$ with length $n$. Suppose $u \in G(Y)$ has length $n+1$, so $u=\zeta v$, where $\zeta \in Y \bigcup Y^{-1}$, and $v \in G(Y)$ has length $n$. One has

$$
\begin{aligned}
{ }^{a} u & ={ }^{a}(\zeta v) & & \\
& =\left[{ }^{a} \zeta\right]\left[a^{\zeta} v\right] & & : \text { by ML2 } \\
& \in G(Y) & & : \text { since }{ }^{a} \zeta, \quad a^{\zeta} v \in G(Y) \text { by the inductive assumption. }
\end{aligned}
$$

Analogous argument verifies $u^{a} \in G(Y)$. This proves part (ii) of the proposition. The proof of (i) is analogous.

Corollary 4.15. In notation as above let $Y$ be a $G$-invariant subset of $(X, r)$. Then

i) $S(Y)$ is an $r_{S}$-invariant subset of the braided monoid $\left(S, r_{S}\right)$

ii) $G(Y)$ is an $r_{G}$-invariant subset of the braided group $\left(G, r_{G}\right)$

Proof. We shall prove (ii) ((i) is analogous). We know that $\left(G, r_{G}\right)$ is a braided group and $r_{G}$ is defined via the left and right actions on $G=G(X, r)$. So we have

$$
r_{G}(u, v)=\left({ }^{u} v, u^{v}\right) \quad \forall u, v \in G .
$$

By Proposition 4.13 each pair $u, v \in G(Y)$ satisfies ${ }^{u} v, u^{v} \in G(Y)$. This shows that $G(Y)$ is $r_{G}$-invariant.

Theorem 4.16. Let $(X, r)$ be a square-free solution, which decomposes into a disjoint union $X=Y \bigcup Z$ of $r$-invariant subsets. Let $\left(Y, r_{Y}\right),\left(Z, r_{Z}\right)$ be the restricted solutions, $G=G(X, r), G_{Y}=G\left(Y, r_{Y}\right), G_{Z}=G\left(Z, r_{Z}\right)$. Then

(1) $G(Y) \simeq G_{Y}, G(Z) \simeq G_{Z}$.

(2) $G_{Y}, G_{Z}$ is a matched pair of groups with actions induced from the braided group $\left(G, r_{G}\right) . G=G(X, r)$ is isomorphic to the double crossed products

$$
G \simeq G_{Y} \bowtie G_{Z} \simeq G_{Z} \bowtie G_{Y} .
$$

In particular, $G$ factorises as:

$$
G=G(X, r)=G_{Y} G_{Z}=G_{Z} G_{Y} .
$$


(3) $\mathcal{G}$ decomposes as product of subgroups (which in general is not a factorisation):

$$
\mathcal{G}=\mathcal{G}(Y) \mathcal{G}(Z)=\mathcal{G}(Z) \mathcal{G}(Y)
$$

Proof. It follows from GIM08 Proposition 4.25. that $G_{Y}, G_{Z}$ is a matched pair, so there is a factorisation $G=G_{Y} G_{Z}$, and each $w \in G$ has unique presentation as

$$
w=u a \quad \text { with } \quad u \in G_{Y}, a \in G_{Z} .
$$

On the other hand $(X, r)$ is a solution, thus $\left(G, r_{G}\right)$ is a braided group and the equality

$$
u a=\left({ }^{u} a\right)\left(u^{a}\right) \text { holds } \forall u, a \in G .
$$

$Y$ and $Z$ are $G$-invariant subsets of $X$, so by Proposition 4.13

$$
\begin{gathered}
a \in G_{Z}, u \in G \Longrightarrow{ }^{u} a \in G_{Z} \\
u \in G_{Y}, a \in G \Longrightarrow u^{a} \in G_{Y} .
\end{gathered}
$$

Therefore each element $w \in G$ presents as

$$
w=u a=a_{1} u_{1} \quad \text { where } \quad u, u_{1} \in G_{Y}, a, a_{1} \in G_{Z}, a_{1}={ }^{u} a, u_{1}=u^{a} .
$$

The uniqueness of $a_{1}$ and $u_{1}$ in (4.11) follows from $G_{Y} \cap G_{Z}=1$. This implies the factorisation $G=G_{Z} G_{Y}$, hence $G_{Y}, G_{Z}$ is a strong matched pair.

We apply the group homomorphism $\mathcal{L}$ to (4.8). Now the equalities $\mathcal{L}(G)=\mathcal{G}$, $\mathcal{L}\left(G_{Y}\right)=\mathcal{L}(G(Y))=\mathcal{G}(Y)$ and $\mathcal{L}\left(G_{Z}\right)=\mathcal{L}(G(Z))=\mathcal{G}(Z)$ give the decomposition (4.9). Note that each $w \in \mathcal{G}$ decomposes as a product $w=u a=a_{1} u_{1}$, where $u, u_{1} \in \mathcal{G}(Y), a, a_{1} \in \mathcal{G}(Z)$, but this presentation is possibly not unique.

Proposition 4.17. With the assumptions and notation of Theorem 4.16, there are isomorphisms of monoids $S(Y) \simeq S\left(Y, r_{Y}\right), \quad S(Z) \simeq S\left(Z, r_{Z}\right) . S(X) \cap S(Y)=1$. Furthermore, $(S(Y), S(Z))$ is a strong matched pair of monoids, $S$ is isomorphic to the double crossed product

$$
S=S(X, r) \simeq S(Y) \bowtie S(Z) \simeq S(Z) \bowtie S(Y) .
$$

There is a factorisation of monoids

$$
S=S(Y) S(Z)=S(Z) S(Y),
$$

where each $w \in S$ decomposes uniquely as

$$
w=u a=a_{1} u_{1}, \quad u, u_{1} \in S(Y), a, a_{1} \in S(Z) .
$$

The following lemma is straighforward. It verifies the associativity of bicross products for $\mathcal{G}$-invariant subset of $(X, r)$.

Lemma 4.18. Notation as above. The double cross product on $\mathcal{G}$-invariant disjoint subsets of $(X, r)$ is commutative and associative. More precisely, suppose $Y_{1}, Y_{2}, Y_{3}$ are pairwise disjoint $\mathcal{G}$-invariant subsets of $X$. Let $Y=Y_{1} \cup Y_{2} \cup Y_{3}$. Then

$$
S\left(Y_{i} \bigcup Y_{j}\right) \simeq S\left(Y_{i}\right) \bowtie S\left(Y_{j}\right) \simeq S\left(Y_{j}\right) \bowtie S\left(Y_{i}\right), 1 \leq i<j \leq s .
$$

Analogous statement is true for the groups $G\left(Y_{i} \bigcup Y_{j}\right), 1 \leq i<j \leq s$. Furthermore,

$$
\begin{aligned}
& S(Y) \simeq S\left(Y_{1}\right) \bowtie\left[S\left(Y_{2}\right) \bowtie S\left(Y_{3}\right)\right] \simeq\left[S\left(Y_{1}\right) \bowtie S\left(Y_{2}\right)\right] \bowtie S\left(Y_{3}\right) \\
& G(Y) \simeq G\left(Y_{1}\right) \bowtie\left[G\left(Y_{2}\right) \bowtie G\left(Y_{3}\right)\right] \simeq\left[G\left(Y_{1}\right) \bowtie G\left(Y_{2}\right)\right] \bowtie G\left(Y_{3}\right) .
\end{aligned}
$$


Theorem 4.19. Let $(X, r)$ be a nontrivial square-free solution. Suppose $X$ decomposes into a disjoint union

$$
X=\bigcup_{1 \leq i \leq p} Y_{i}
$$

of $\mathcal{G}$-invariant subsets $Y_{1}, Y_{2}, \cdots Y_{s}$. Then

$$
\begin{gathered}
S=S(X, r) \simeq S\left(Y_{1}\right) \bowtie S\left(Y_{2}\right) \bowtie \cdots S\left(Y_{s}\right) \\
G=G(X, r) \simeq G\left(Y_{1}\right) \bowtie G\left(Y_{2}\right) \bowtie \cdots G\left(Y_{s}\right) .
\end{gathered}
$$

In particular,

(1) $S=S(X, r)$ factorises as a product of submonoids:

$$
S=S(X, r)=S\left(Y_{1}\right) S\left(Y_{2}\right) \cdots S\left(Y_{s}\right),
$$

where each $u \in S$ has unique presentation $u=u_{1} u_{2} \cdots u_{s}, u_{i} \in S\left(Y_{i}\right), 1 \leq$ $i \leq s$.

(2) $G=G(X, r)$ factorises as a product of subgroups:

$$
G=G(X, r)=G\left(Y_{1}\right) G\left(Y_{2}\right) \cdots G\left(Y_{s}\right),
$$

where each $u \in G$ has unique presentation $u=u_{1} u_{2} \cdots u_{s}, u_{i} \in G\left(Y_{i}\right), 1 \leq$ $i \leq s$.

$$
\mathcal{G}=\mathcal{G}(X, r)=\mathcal{G}\left(Y_{1}\right) \mathcal{G}\left(Y_{2}\right) \cdots \mathcal{G}\left(Y_{s}\right)
$$

in the sense that each $a \in \mathcal{G}$ is presented as a product $a=a_{1} a_{2} \cdots a_{s}$, where $a_{i} \in \mathcal{G}\left(Y_{i}\right), 1 \leq i \leq s$, but this presentation is possibly not unique. At least one of the groups $\mathcal{G}\left(Y_{i}\right)$ is nontrivial. (We have $\mathcal{G}\left(Y_{i}\right)=1$ if and only if $Y_{i} \subset \operatorname{ker} \mathcal{L}$ ).

Corollary 4.20. Let $(X, r)$ be a nontrivial square-free solution, which is either finite, or infinite but with a finite set of $\mathcal{G}$-orbits. Let $X_{1} \cdots X_{t}$ be the set of all orbits in $X$ denoted so that the first $t_{0}$ orbits are exactly the nontrivial ones. Then

$$
\begin{aligned}
& S=S(X, r)=S\left(X_{1}\right) S\left(X_{2}\right) \cdots S\left(X_{t}\right) \\
& G=G(X, r)=G\left(X_{1}\right) G\left(X_{2}\right) \cdots G\left(X_{t}\right) \\
& \mathcal{G}=\mathcal{G}(X, r)=\mathcal{G}\left(X_{1}\right) \mathcal{G}\left(X_{2}\right) \cdots \mathcal{G}\left(X_{t_{0}}\right) \text {. }
\end{aligned}
$$

For multipermutation square-free solutions $(X, r)$ with $m p l X=m$ there is a natural and important decomposition: $X$ decomposes as a disjoint union of its $(m-1)$ th retract classes. The retract classes $\left[x^{(k)}\right], 1 \leq k, x \in X$ are introduced in section 7 see Notation 7.1, and Facts 7.4. They are disjoint $r$-invariant subsets of $(X, r)$ and behave nicely. Note that when $\operatorname{mpl} X=m$, and $k<m-1$ at least one of the $k$-th retract classes is not $\mathcal{G}$-invariant. Moreover, each $(m-1)$-th retract class $\left[x^{(m-1)}\right]$ is $\mathcal{G}$-invariant and contains the orbit $\mathcal{O}_{\mathcal{G}}(x)$. More precisely, each retract class $\left[x^{(m-1)}\right]$ splits into a disjoint union of the orbits $X_{i_{j}}$, which intersect it nontrivially.

Theorem 4.21. Let $(X, r)$ be a nontrivial square-free solution, which is either finite, or is infinite but with a finite number of $\mathcal{G}$-orbits. Suppose it has a finite multipermutation level $\operatorname{mpl}(X, r)=m$. Then

(1) $\operatorname{Ret}^{(m-1)}(X, r)$ is a finite set of order $\leq t$, where $t$ is the number of $\mathcal{G}$-orbits of $X$. Let

$$
Y_{1}=\left[\xi_{1}^{(m-1)}\right], \cdots, Y_{s}=\left[\xi_{s}^{(m-1)}\right]
$$


be the set of all distinct $(m-1)$-retract classes in $X$. One has $s \geq 2$, and $\left|Y_{i}\right| \geq 2$ for some $1 \leq i \leq s$.

(2) $X$ is a disjoint union $X=\bigcup_{1 \leq i \leq s} Y_{i}$. Each $Y_{i}, 1 \leq i \leq s$, is $\mathcal{G}$-invariant, and $\operatorname{mpl}\left(Y_{i}, r_{i}\right) \leq m-1$, where $\left(\bar{Y}_{i}, r_{i}\right)$ is the restricted solution.

(3) The monoid $S=S(X, r)$ and the group $G=G(X, r)$ have factorisations as in (4.12) and (4.13), respectively. $\mathcal{G}=\mathcal{G}(X, r)$ also decomposes as a product of subgroups (4.14), but some pairs of these subgroups may have nontrivial intersection.

Proof. Clearly $X=\bigcup_{1 \leq i \leq s} Y_{i}$ is a disjoint union. It follows from Proposition 7.8 that $s \geq 2, Y_{i}$ is $\mathcal{G}$-invariant for $1 \leq i \leq s$ and contains each $\mathcal{G}$-orbit which intersects it nontrivially. If we assume $\left|Y_{i}\right|=1,1 \leq i \leq s$, this would imply that all $\mathcal{G}$ - orbits in $X$ are trivial, and therefore $(X, r)$ is a trivial solution, a contradiction. It follows than that $\left|Y_{i}\right| \geq 2$ for some $i, 1 \leq i \leq s$. The inequality $\operatorname{mpl}\left(Y_{i}, r_{i}\right) \leq m-1$ follows from Facts 7.4 2, We have proved (1) and (2). (3) follows straightforwardly from Theorem 4.19

Remark 4.22. Proposition 5.15 shows that in the particular case when $2 \leq m \leq 3$ $(X, r)$ is a strong twisted union

$$
X=Y_{1} \curvearrowleft Y_{2} \downarrow \cdots \curvearrowleft Y_{s},
$$

and $\mathcal{G}$ decomposes as a product of abelian subgroups

$$
\mathcal{G}=\mathcal{G}\left(Y_{1}\right) \mathcal{G}\left(Y_{2}\right) \cdots \mathcal{G}\left(Y_{s}\right)
$$

\section{Multipermutation solutions of low Levels.}

A natural question arises:

Question 5.1. Suppose $(X, r)$ is a multipermutation square-free solution. What is the relation between the multipermutation level $\operatorname{mpl}(X, r)$ and the algebraic properties of $S(X, r), G(X, r), \mathcal{G}(X, r), k \mathcal{G}(X, r), \mathcal{A}(k, X, r)$ ?

The following is straightforward.

Lemma 5.2. Suppose $(X, r)$ is a square-free solution of order $\geq 2$ (but not necessarily finite). Then the following conditions are equivalent.

(1) $\operatorname{mpl}(X, r)=1$.

(2) $(X, r)$ is the trivial solution, i.e. $r(x, y)=(y, x)$, for all $x, y \in X$.

(3) $S(X, r)$ is the free abelian monoid generated by $X$.

(4) $G(X, r)$ is the free abelean group generated by $X$.

(5) $\mathcal{G}(X, r)=\{$ id $\}$, the trivial group.

5.1. Square-free solutions of multipermutation level 2. Detailed study of square-free solutions of multipermutation level 2 is performed in GIM0806, and GIM08. We recall first some results from these works needed in the sequel.

Proposition 5.3. GIM0806, Let $(X, r)$ be a square-free solution of finite order, let $X_{i}, 1 \leq i \leq t$ be the set of all $G(X, r)$-orbits in $X$ enumerated so that $X_{1}, \cdots, X_{t_{0}}$ is the set of all nontrivial orbits (if any). Then the following are equivalent. 
(1) $(X, r)$ is a multipermutation solution of level 2

(2) $t_{0} \geq 1$ and for each $j, 1 \leq j \leq t_{0}, x, y \in X_{j}$ implies $\mathcal{L}_{x}=\mathcal{L}_{y}$.

(3) $t_{0} \geq 1$ and for each $x \in X$ the permutation $\mathcal{L}_{x}$ is an r-automorphism, i.e. $\mathcal{G}(X, r) \subseteq \operatorname{Aut}(X, r)$.

Theorem 5.4. GIM0806 Let $(X, r)$ be square-free solution of multipermutation level 2 and finite order, and $X_{i}, 1 \leq i \leq t$ be the set of all $G(X, r)$-orbits as in Popostion 5.3. Let $\left(X_{i}, r_{i}\right), 1 \leq i \leq t$ be the restricted solution. Then:

(1) $\mathcal{G}(X, r)$ is a nontrivial abelian group.

(2) Each $\left(X_{i}, r_{i}\right), 1 \leq i \leq t_{0}$ is a trivial solution. Clearly in the case $t_{0}<t$, each $\left(X_{j}, r_{j}\right)$, with $t_{0} \leq j \leq t$ is a one element solution.

(3) For any ordered pair $i, j, 1 \leq i \leq t_{0}, 1 \leq j \leq t$, such that $X_{j}$ acts nontrivially on $X_{i}$, every $x \in X_{j}$ acts via the same permutation $\sigma_{i}^{j} \in \operatorname{Sym}\left(X_{i}\right)$ which is a product of disjoint cycles of equal length $d=d_{i}^{j}$

$$
\sigma_{i}^{j}=\left(x_{1} \cdots x_{d}\right)\left(y_{1} \cdots y_{d}\right) \cdots\left(z_{1} \cdots z_{d}\right),
$$

where each element of $X_{i}$ occurs exactly once. Here $d_{i}^{j}$ is an invariant of the pair $X_{j}, X_{i}$.

(4) $X$ is a strong twisted union $X=X_{1} \downarrow X_{2} \downarrow \cdots \downarrow X_{t}$.

Fact 5.5. GIM08, Proposition 2.5. Let $(X, r)$ be a quadratic set. Then any of the following two conditions imply the third. (i) $(X, r)$ is involutive; (ii) $(X, r)$ is nondegenerate and cyclic; (iii) $(X, r)$ satisfies lri.

Proposition 5.6. Suppose $(X, r)$ is a multipermutation solution of level 2. Then

(1) The associated braided monoid $\left(S, r_{S}\right)$ is a symmetric set which satisfies the cyclic conditions and lri (see Definition [2.9). (S, $\left.r_{S}\right)$ is not square-free. Furthermore, $S$ acts on itself as automorphisms:

$$
{ }^{a}(u v)=\left({ }^{a} u\right)\left({ }^{a} v\right) \quad(u v)^{a}=\left(u^{a}\right)\left(v^{a}\right) \quad \forall \quad a, u, v \in S .
$$

(2) An analogous statement is true for the associated braided group $\left(G, r_{G}\right)$.

Proof. By Facts 4.6 $\left(S, r_{S}\right)$ is an nondegenerate involutive set-theoretic solution of YBE, therefore it is a symmetric set. By Proposition $5.3 \mathcal{G}(X, r) \subseteq \operatorname{Aut}(X, r)$, thus, by Corollary 3.5 and by the definition of automorphism of solutions one has

$$
\begin{array}{cl}
\mathcal{L}^{\alpha} x=\mathcal{L}_{x}=\mathcal{L}_{x^{\alpha}} & \forall x, \alpha \in X \\
{ }^{a}(x y)=\left({ }^{a} x\right)\left({ }^{a} y\right) & \forall a \in S, x, y \in X .
\end{array}
$$

Using (5.2) and induction on the length $|v|$ of $v \in S$ ) one shows easily that

$$
\begin{aligned}
& a^{b} v={ }^{a} v \quad v^{b}=v^{a}, \quad \text { for all } a, b, v \in S \text {; } \\
& { }^{b} a={ }^{a} v, \quad v^{a^{b}}=v^{a} \quad \text { for all } a, b, v \in S \text {. }
\end{aligned}
$$

replacing $b$ with $v$ in each of the above equalities one yields the cyclic conditions for the solution $\left(S, r_{S}\right)$. We have shown that $\left(S, r_{S}\right)$ satisfies (i) and (ii) of Fact 5.5. hence it satisfies (iii). This verifies lri $\left(\left({ }^{a} u\right)^{a}=u={ }^{a}\left(u^{a}\right)\right.$ for all $\left.a, u \in S\right)$. We will verify that the left action of $S$ (see Facts 4.6) is as automorphisms (the proof for the right action is analogous). This follows from the equalities:

$$
\begin{aligned}
{ }^{a}(u v) & =\left({ }^{a} u\right)\left({ }^{a^{u}} v\right) & & \text { : by ML2 } \\
& =\left({ }^{a} u\right)\left({ }^{a} v\right) & & \text { : by (5.3). }
\end{aligned}
$$


We claim that $\left(S, r_{S}\right)$ is not square-free, or equivalently there exist an $a \in S$, such that ${ }^{a} a \neq a$. Assume the contrary. By hypothesis $(X, r)$ is not the trivial solution, so there exist $x, y \in X$, with ${ }^{y} x \neq x$. Let $a=x y$

$$
\begin{aligned}
{ }^{a} a={ }^{x y}(x y) & =\left({ }^{x y} x\right)\left({ }^{x y} y\right) & & : \text { by }(5.1) \\
& =\left({ }^{y}\left({ }^{x} x\right)\right)\left({ }^{x}\left({ }^{y} y\right)\right) & & : \text { by } \mathcal{G} \text { abelian } \\
& =\left({ }^{y} x\right)\left({ }^{x} y\right) & & : \text { by }(X, r) \quad \text { square-free. }
\end{aligned}
$$

So $x y=a={ }^{a} a=\left({ }^{y} x\right)\left({ }^{x} y\right)$ are equalities in $S$. The only quadratic relation in $S$ involving $x y$ is

$$
x y={ }^{x} y \cdot x^{y} .
$$

Therefore one of the following is an equality of words in the free monoid $\langle X\rangle$ :

$$
\begin{gathered}
\left({ }^{y} x\right)\left({ }^{x} y\right)=x y \quad \text { in } \quad\langle X\rangle \\
\left({ }^{y} x\right)\left({ }^{x} y\right)={ }^{x} y \cdot x^{y} \quad \text { in } \quad\langle X\rangle .
\end{gathered}
$$

Case a. (5.4) holds. Then ${ }^{y} x=x$ which contradicts the choice of $x$ and $y$. Case b. (5.5) is in force. Hence ${ }^{y} x={ }^{x} y$. We apply right action by ${ }^{y}$ on both sides of this equality and obtain

$$
\left({ }^{y} x\right)^{y}=\left({ }^{x} y\right)^{y} .
$$

Thus

$$
\begin{array}{rlrl}
x & =\left({ }^{y} x\right)^{y} & \text { by } \mathbf{l r i} \\
& =\left({ }^{x} y\right)^{y} & \text { by }(\sqrt[5.6)]{ } \\
& ={ }^{x}\left(y^{y}\right) & \text { by } \mathcal{G} \text { abelian } \\
& ={ }^{x} y & \text { by }(X, r) & \text { square-free. }
\end{array}
$$

Therefore ${ }^{x} y=x={ }^{x} x$. It follows then by the nondegeneracy of $(X, r)$ that $y=x$, and ${ }^{y} x={ }^{y} y=y$. A contradiction with the choice of $x, y$. We have verified the first part of the proposition. An analogous argument proves the statement for the braided group $\left(G, r_{G}\right)$.

It is natural to ask

Questions 5.7. 1) When can an abelian group of permutations $H \leq \operatorname{Sym} X$ be considered as a permutation YB group of a solution $(X, r)$ ?

In particular,

2) When is $H \leq \operatorname{Sym} X$ a permutation $Y B$ group of a solution $(X, r)$ of multipermutation level 2?

An answer to the second question is given in the following Proposition 5.8 Furthermore, as shows Corollary [5.9, every finite abelian group is isomorphic to the permutation group of a solution $(X, r)$ with $\operatorname{mpl} X=2$.

Proposition 5.8. Let $H$ be an abelian permutation group on a set $X$. Then the following are equivalent:

(a) there is a solution $(X, r)$, with $\operatorname{mpl}(X, r)=2$, such that $\mathcal{G}(X, r)=H$;

(b) there is a function $f$ from the set of $H$-orbits on $X$ to $H$ with the properties

- $f\left(X_{i}\right)$ fixes every point in $X_{i}$;

- the image of $f$ generates $H$. 
Proof. We use the following facts: If $H$ is an abelian permutation group on $X$, and if $h \in H$ fixes $x \in X$, then $H$ fixes every point of the $H$-orbit containing $X$. For, if $k \in H$, then

$$
h(k(x))=k(h(x))=k(x) .
$$

Also, if a solution $(X, r)$ has $\operatorname{mpl}(X, r)=2$, and $\mathcal{L}_{a}(x)=y$, then $\mathcal{L}_{x}=\mathcal{L}_{y}$. For in the first retract, $\mathcal{L}_{[a]}$ is the identity, so that $[x]=[y]$, which means precisely that $\mathcal{L}_{x}=\mathcal{L}_{y}$. It follows that if $x$ and $y$ lie in the same orbit of $\mathcal{G}(X, r)$, then $\mathcal{L}_{x}=\mathcal{L}_{y}$.

Now suppose that $H=\mathcal{G}(X, r)$ for some solution $(X, r)$ with $\operatorname{mpl}(X, r)=2$. For any orbit $X_{i}$, choose $a \in X_{i}$, and let $f\left(X_{i}\right)=\mathcal{L}_{a}$. This element fixes $a$, and hence fixes every point of its orbit. Moreover, the previous paragraph shows that $f\left(X_{i}\right)$ is independent of the choice of $a \in X_{i}$. Also, the image of $f$ consists of all permutations $\mathcal{L}_{a}$, for $a \in X$; so it generates $\mathcal{G}(X, r)=H$.

Conversely, suppose that we are given a function $f$ with the properties in the proposition. For all $a \in X_{i}$, we define $\mathcal{L}_{a}=f\left(X_{i}\right)$, and then construct a map $r: X \times X \rightarrow X \times X$ in the usual way:

$$
r(a, b)=\left(\mathcal{L}_{a}(b), \mathcal{L}_{b}^{-1}(a)\right) .
$$

By assumption, $\mathcal{L}_{a}(a)=a$. Also, for any $a, b \in X, \mathcal{L}_{a}(b)={ }^{a} b$ lies in the same orbit as $b$, and hence $\mathcal{L}_{a b}=\mathcal{L}_{b}$; similarly $\mathcal{L}_{a^{b}}=\mathcal{L}_{a}$; and the fact that the group is abelian now implies that

$$
\mathcal{L}_{a} \mathcal{L}_{a^{b}}=\mathcal{L}_{b} \mathcal{L}_{a}=\mathcal{L}_{a} \mathcal{L}_{b}
$$

It follows that we do have a solution. Moreover, the group $H$ is generated by all the maps $\mathcal{L}_{a}$, for $a \in X$; so $H=\mathcal{G}(X, r)$.

From this we can deduce the following.

Corollary 5.9. Let $H$ be a finite abelian group. Then there is a solution $(X, r)$ with $\operatorname{mpl}(X, r)=2$ such that $\mathcal{G}(X, r) \cong H$.

Proof. Let $h_{1}, \ldots, h_{r}$ generate $H$. Now let $X=H \cup\left\{a_{1}, \ldots, a_{r}\right\}$, where $H$ has its regular action on itself and fixes the points $a_{1}, \ldots, a_{r}$. Define a function $f$ by

$$
\begin{aligned}
f(H) & =\mathrm{id}, \\
f\left(\left\{a_{i}\right\}\right) & =h_{i} .
\end{aligned}
$$

The conditions of the proposition are obviously satisfied by $f$.

5.2. Square-free solutions of multipermutation level 3. It is straightforward that $\mathrm{mpl} X>2$ if and only if $\operatorname{Ret}(X, r)$ is a nontrivial solution, or equivalently

$$
\exists \alpha, x \in X, \text { such that }\left[{ }^{\alpha} x\right] \neq[x] .
$$

Remark 5.10. The following are equivalent

i) $\operatorname{mpl}(X, r)>2$

ii) There exists a first-retract class $[x]=\left[x^{(1)}\right]$ which is not $\mathcal{G}$-invariant.

(iii) There exists a $\mathcal{G}$-orbit $X_{0}$ in $X$ and a pair $\alpha, \beta \in X_{0}$ such that $\mathcal{L}_{\alpha} \neq \mathcal{L}_{\beta}$.

Proposition 5.11. Let $(X, r)$ be a square-free solution (of arbitrary cardinality). 
(1) $\operatorname{mpl}(X, r) \leq 3$ if and only if the following condition holds

$$
\mathcal{L}_{\left({ }^{\beta} x\right)}=\mathcal{L}_{\left({ }^{\alpha} x\right)} \quad \forall \alpha, \beta, x \in X \quad \text { with } \quad \mathcal{O}_{\mathcal{G}}(\alpha)=\mathcal{O}_{\mathcal{G}}(\beta) .
$$

(2) $\mathrm{mpl} X=3$, if and only if (5.7) holds, and there exists a pair $x, \alpha \in X$ such that

$$
\mathcal{L}_{\left({ }^{\alpha} x\right)} \neq \mathcal{L}_{x}
$$

Proof. The following implications hold:

$\operatorname{mpl}(X, r) \leq 3 \Longleftrightarrow \operatorname{Ret}^{2}(X, r) \quad$ is either the trivial solution or one element solution $\Longleftrightarrow[\alpha] \sim\left[{ }^{u} \alpha\right] \quad$ in $\operatorname{Ret}(X, r) \quad \forall \alpha \in X, u \in \mathcal{G}$

$\Longleftrightarrow\left[{ }^{\alpha} x\right]=\left[{ }^{\beta} x\right] \quad \forall \alpha, \beta, x \in X$, with $\quad \mathcal{O}_{\mathcal{G}}(\alpha)=\mathcal{O}_{\mathcal{G}}(\beta)$

$\Longleftrightarrow \mathcal{L}_{\alpha_{x}}=\mathcal{L}_{\beta} \quad \forall \alpha, \beta, x \in X$, with $\quad \mathcal{O}_{\mathcal{G}}(\alpha)=\mathcal{O}_{\mathcal{G}}(\beta)$.

This implies the first part of the proposition. Now (1) together Remark 5.10 imply (2).

Proposition 5.12. Let $(X, r)$ be a nontrivial square-free solution with condition (5.7). Suppose $X_{i}, 1 \leq i \leq t$, is the set of all $G(X, r)$-orbits in $X$ enumerated so that $X_{1}, \cdots, X_{t_{0}},\left(t_{0} \geq 1\right)$ are exactly the nontrivial ones. Then the following are equivalent. Then the following conditions hold:

(1) There are equalities

$$
{ }^{\beta} x={ }^{x} \alpha \quad{ }^{y} x={ }^{\alpha} x \quad \forall x, y \in X_{i}, \forall \alpha, \beta \in X_{j}, 1 \leq i<j \leq t .
$$

(2) Each group $\mathcal{G}\left(X_{j}\right), 1 \leq j \leq t_{0}$, is abelian. In the case when $t_{0}<t, \mathcal{G}\left(X_{j}\right)=$ $\{1\}$, for all $t_{0}<j \leq t$.

(3) $X$ is a strong twisted union $X=X_{1} \natural X_{2} \natural \cdots \natural X_{t}$.

(4) In particular, $\operatorname{mpl}(X, r)=3$ implies (5.7) and conditions (1), (2), (3).

Proof. We apply the two sides of (5.7) to the element $\alpha$, and use the cyclic condition to yield: $\mathcal{L}_{\beta}(\alpha)=\mathcal{L}_{\alpha_{x}}(\alpha)=\mathcal{L}_{x}(\alpha)$. This interpreted in our typical notation gives:

$$
{ }^{\beta} x \alpha={ }^{x} \alpha \quad \forall x \in X, \alpha, \beta \in X_{j}, 1 \leq j \leq t,
$$

We have verified the left hand-side equality of (5.8). The right hand-side is analogous. This proves part (1).

To prove (3) it will be enough to show that for two arbitrary orbits $X_{i}, X_{j}, 1 \leq i<$ $j \leq t$ the set $X_{i j}=X_{i} \bigcup X_{j}$ is $r$-invariant, and the restricted solution $\left(X_{i j}, r_{\mid X_{i j}}\right)$ is a strong twisted union $X_{i j}=X_{i} \curvearrowleft X_{j}$.

Consider two orbits $X_{i}, X_{j}$, where $1 \leq i<j \leq t$. As a union of two $\mathcal{G}$-invariant subsets of $X$, the set $X_{i j}=X_{i} \bigcup X_{j}$ is $r$-invariant, so (5.8) implies that the restricted solution $\left(X_{i j}, r_{\mid X_{i j}}\right)$ is a strong twisted union $X_{i j}=X_{i} \natural X_{j}$, which proves (3).

Suppose now $1 \leq j \leq t$. Without loss of generality we can assume that the group $\mathcal{G}\left(X_{j}\right)$ is nontrivial (by hypothesis $(X, r)$ is a nontrivial solution). Now in the equality (5.7) we set $x=\alpha$ and since $(X, r)$ is square-free $\left({ }^{\alpha} \alpha=\alpha\right)$ we obtain the left hand-side of the following

$$
\mathcal{L}_{\beta}{ }=\mathcal{L}_{\alpha}, \quad \mathcal{L}_{\beta^{\alpha}}=\mathcal{L}_{\beta} \quad \forall \alpha, \beta \in X_{j}, \quad 1 \leq j \leq t .
$$


The equality in the right hand-side is analogous. Recall that since $(X, r)$ is a solution one has

$$
\mathcal{L}_{\beta} \circ \mathcal{L}_{\alpha}=\mathcal{L}_{\beta} \circ \mathcal{L}_{\beta^{\alpha}} \quad \forall \alpha, \beta \in X,
$$

which together with (5.9) implies

$$
\mathcal{L}_{\beta} \circ \mathcal{L}_{\alpha}=\mathcal{L}_{\alpha} \circ \mathcal{L}_{\beta}, \quad \forall \alpha, \beta \in X_{j}, \quad 1 \leq j \leq t .
$$

By definition, $\mathcal{G}\left(X_{j}\right)$ is the subgroup of $\mathcal{G}$, generated by the set of all $\mathcal{L}_{\alpha}, \alpha \in X_{j}$. It follows then from (5.10) that each nontrivial $\mathcal{G}\left(X_{j}\right), 1 \leq j \leq t$, is abelian.

Corollary 5.13. Let $(X, r)$ be a finite square-free solution with $\mathrm{mpl} X=3$. Let $X_{1} \cdots X_{t}$ be the set of $\mathcal{G}$-orbits in $X$. Then

(1) Each group $\mathcal{G}\left(X_{j}\right), 1 \leq j \leq t$, is abelian $\left(\mathcal{G}\left(X_{j}\right)=\{e\}\right.$ is also possible). There exists a $j, 1 \leq j \leq t$ for which $\mathcal{G}\left(X_{j}\right)$ is nontrivial. Without loss of generality we shall assume that the nontrivial groups are $\mathcal{G}\left(X_{j}\right)$ with $1 \leq j \leq t_{0}$

(2) Suppose $1 \leq j \leq t_{0}$, so $\mathcal{G}\left(X_{j}\right)$ acts on $X$ nontrivially. Consider the set of $\mathcal{G}\left(X_{j}\right)$-orbits in $X$. Then the elements of each $\mathcal{G}\left(X_{j}\right)$-orbit in $X$ act equally on $X_{j}$, that is:

$$
\left(\mathcal{L}_{\alpha}\right)_{\mid X_{j}}=\left(\mathcal{L}_{\beta}\right)_{\mid X_{j}} \quad \text { whenever } \quad \beta \in \mathcal{O}_{\mathcal{G}\left(X_{j}\right)}(\alpha) .
$$

Moreover, $X=X_{1} \natural X_{2} \natural \cdots \curvearrowleft X_{t}$.

(3) The group $\mathcal{G}$ is a product of abelian subgroups

$$
\mathcal{G}=\mathcal{G}\left(X_{1}\right) \mathcal{G}\left(X_{2}\right) \cdots \mathcal{G}\left(X_{t_{0}}\right) .
$$

This corollary gives interesting information about the graph $\Gamma$ of finite solutions $(X, r)$ with $\operatorname{mpl} X=3$. Take an arbitrary $j$ for which the group $\mathcal{G}\left(X_{j}\right)$ is nontrivial. The action of $\mathcal{G}\left(X_{j}\right)$ on $X$ is represented graphically by taking the subgraph $\Gamma^{(j)}$ of $\Gamma$ with the same set of vertices, but only edges labeled by $\alpha$, where $\alpha \in X_{j}$, are considered. Then clearly, there is a $1-1$ correspondence between the set of connected components in $\Gamma^{(j)}$ and the set of $\mathcal{G}\left(X_{j}\right)$-orbits in $X$. For each component $\Gamma_{x}^{(j)}(x$ is an arbitrary vertex in the component) the corresponding orbit is $\mathcal{O}_{\mathcal{G}\left(X_{j}\right)}(x)$. As a set it coincides with the set of all vertices in $\Gamma_{x}^{(j)}$. It follows then from Corollary 5.13 that all vertices in a connected component of $\Gamma^{(j)}, 1 \leq j \leq t_{0}$ have the same action on the set $X_{j}$.

Question 5.14. In notation as above, suppose that $(X, r)$ is a finite square-free solution which satisfies condition (1) and (2) of Corollary 5.13. What additional "minimal" condition should be imposed on the actions (if any) to guarantee $\mathrm{mpl} X=$ 3? We are searching a condition which formally is weaker that the obviously suffcient condition (5.7).

Proposition 5.15. Let $(X, r)$ be a nontrivial finite square-free solution of multipermutation level $m, 2 \leq m \leq 3$. Let

$$
Y_{1}=\left[\xi_{1}^{(m-1)}\right], \cdots, Y_{s}=\left[\xi_{s}^{(m-1)}\right]
$$

be the set of all distinct $(m-1)$-retract classes in $X$. Let $S\left(Y_{j}\right), G\left(Y_{j}\right), \mathcal{G}\left(Y_{j}\right), 1 \leq$ $j \leq s$, be as in Notation 4.10. Then

(1) $s \geq 2,\left|Y_{i}\right| \geq 2$ for some $1 \leq i \leq s$. (We enumerate them so that the first $s_{0}$ are exactly the nontrivial ones). 
(2) Each retract class $Y_{i}, 1 \leq i \leq s$, is $\mathcal{G}$-invariant and the restricted solution $\left(Y_{i}, r_{i}\right)$ where $r_{i}=r_{\mid Y_{i}}$ has multipermutation level $\leq 2$. (In the case when $\left.s_{0}<i \leq s, \operatorname{mpl} Y_{j}=0\right)$.

(3) $(X, r)$ is a strong twisted union

$$
X=Y_{1} \curvearrowleft Y_{2} \curvearrowleft \cdots \curvearrowleft Y_{s} .
$$

(4) All groups $\mathcal{G}\left(Y_{j}\right), 1 \leq j \leq s$, are abelian and $\mathcal{G}\left(Y_{j}\right)=1$ is possible for at most one retract class $Y_{j}$ (with $Y_{j} \subset \operatorname{ker} \mathcal{L}$ ). Furthermore,

$$
\mathcal{G}=\mathcal{G}\left(Y_{1}\right) \mathcal{G}\left(Y_{2}\right) \cdots \mathcal{G}\left(Y_{s_{0}}\right) .
$$

Proof. We give a sketch of the proof for the case $\operatorname{mpl} X=3$, the case $\operatorname{mpl} X=2$ is analogous. 1 is clear. 2 follows from Facts 7.42 We shall prove 3 . Clearly, $X$ splits into a disjoint union $X=\bigcup_{1 \leq j \leq s} Y_{j}$. of the $\mathcal{G}$-invariant subsets $Y_{j}, 1 \leq j \leq s$. By Definition 3.8 it will be enough to show that for each pair $i \neq j, 1 \leq i, j \leq s$ the $\mathcal{G}$-invariant subset $Y_{i j}=Y_{i} \bigcup Y_{j}$ is a strong twisted union $Y_{i j}=Y_{i} \curvearrowleft Y_{j}$. Suppose $\alpha, \beta \in Y_{i}$, then $\alpha^{(2)}=\beta^{(2)}$ and by Facts 7.4, (17.3) one has

$$
{ }^{\alpha}{ }^{x} \beta={ }^{x} \beta \quad \forall x \in X,
$$

which implies $Y_{i j}=Y_{i}\left\lfloor Y_{j}\right.$. This proves 3 ,

By Corollary 7.7 each group $\mathcal{G}\left(Y_{i}\right), 1 \leq$ is abelian. By Remarks $4.11 \mathcal{G}\left(Y_{j}\right)=1$ iff $Y_{j} \subset \operatorname{ker} \mathcal{L}$, and 7.6 implies that if such a retract class exists it contains the set $X_{0}=X \bigcap \operatorname{ker} \mathcal{L}$, and therefore it is unique. Now 4 follows from the decomposition Theorem 4.21 (3).

We show in section 6 that for square-free solutions of arbitrary cardinality and finite multipermutation level $m$ one has

$$
s l \mathcal{G}(X, r) \leq m-1, \quad s l G(X, r) \leq m
$$

Thus

$$
\operatorname{mpl} X=3 \Longrightarrow 1 \leq \operatorname{slG}(X, r) \leq 2 .
$$

We conclude the section with an example of a square-free solutions $(X, r)$ with $\operatorname{mpl}(X, r)=3$ and abelian YB permutation group $\mathcal{G}(X, r)$.

Example 5.16. Let $(X, r)$ be the square-free solution defined as

$$
\begin{gathered}
X=X_{1} \cup X_{2} \cup X_{3} \\
X_{1}=\left\{x_{i} \mid 1 \leq i \leq 8\right\} \quad X_{2}=\{a, c\} \quad X_{3}=\{b, d\} \\
\mathcal{L}_{a}=(b d)\left(x_{1} x_{2}\right)\left(x_{3} x_{4}\right)\left(x_{5} x_{6}\right)\left(x_{7} x_{8}\right) \quad \mathcal{L}_{c}=(b d)\left(x_{1} x_{5}\right)\left(x_{2} x_{6}\right)\left(x_{3} x_{7}\right)\left(x_{4} x_{8}\right) \\
\mathcal{L}_{b}=(a c)\left(x_{1} x_{3}\right)\left(x_{2} x_{4}\right)\left(x_{5} x_{7}\right)\left(x_{6} x_{8}\right) \quad \mathcal{L}_{c}=(a c)\left(x_{1} x_{8}\right)\left(x_{2} x_{7}\right)\left(x_{3} x_{6}\right)\left(x_{5} x_{4}\right) \\
\mathcal{L}_{x_{i}}=\operatorname{id}_{X} \quad 1 \leq i \leq 8 .
\end{gathered}
$$

Then $\operatorname{mpl}(X, r)=3, \mathcal{G}(X, r)$ is abelian, and the group $G(x, r)$ is solvable of solvable length 2. 
Proof. Note that ${ }^{a} b=d, a^{b}=c$, so $\mathcal{L}_{a} \mathcal{L}_{b}=\mathcal{L}_{a_{b}} \mathcal{L}_{a^{b}}$. Similarly one veryfies that $\mathcal{L}_{z} \mathcal{L}_{t}=\mathcal{L}_{z} \mathcal{L}_{z^{t}}$, for all $z, t \in X$, so condition $\mathbf{1 1}$ is satisfied and the left action given above defines a solution. Direct computation shows:

$$
\begin{aligned}
& \mathcal{L}_{a} \circ \mathcal{L}_{b}=\mathcal{L}_{b} \circ \mathcal{L}_{a}=\mathcal{L}_{d} \circ \mathcal{L}_{c}=\mathcal{L}_{c} \circ \mathcal{L}_{d}=(a c)(b d)\left(x_{1} x_{4}\right)\left(x_{2} x_{3}\right)\left(x_{5} x_{8}\right)\left(x_{6} x_{7}\right) \\
& \mathcal{L}_{a} \circ \mathcal{L}_{c}=\mathcal{L}_{c} \circ \mathcal{L}_{a}=\mathcal{L}_{b} \circ \mathcal{L}_{d}=\mathcal{L}_{d} \circ \mathcal{L}_{b}=\left(x_{1} x_{6}\right)\left(x_{2} x_{5}\right)\left(x_{3} x_{8}\right)\left(x_{4} x_{7}\right) \\
& \mathcal{L}_{a} \circ \mathcal{L}_{d}=\mathcal{L}_{d} \circ \mathcal{L}_{a}=\mathcal{L}_{b} \circ \mathcal{L}_{c}=\mathcal{L}_{c} \circ \mathcal{L}_{b}=(a c)(b d)\left(x_{1} x_{7}\right)\left(x_{2} x_{8}\right)\left(x_{3} x_{5}\right)\left(x_{4} x_{6}\right) .
\end{aligned}
$$

Hence $\mathcal{G}=\mathcal{G}(X, r)=\left\langle\mathcal{L}_{a}, \mathcal{L}_{b}, \mathcal{L}_{c}, \mathcal{L}_{d}\right\rangle$ is abelian. Next, the equality $\mathcal{L}_{a b}=\mathcal{L}_{d} \neq \mathcal{L}_{b}$, implies that $\operatorname{mpl}(X, r) \geq 3$. It is easy to see that

$$
\mathcal{G}=\left\langle\mathcal{L}_{a}\right\rangle \times\left\langle\mathcal{L}_{b}\right\rangle \times\left\langle\mathcal{L}_{c}\right\rangle \simeq C_{2} \times C_{2} \times C_{2} .
$$

We will show that $\operatorname{mpl}(X, r)=3$. For the retracts one has

$$
\begin{array}{r}
\operatorname{Ret}(X, r)=\left([X], r_{[X]}\right), \text { where }[X]=\left\{[a],[b],[c],[d],\left[x_{1}\right]\right\}, \\
\mathcal{L}_{[a]}=\mathcal{L}_{[c]}=([b][d]), \mathcal{L}_{[b]}=\mathcal{L}_{[d]}=([a][c])
\end{array}
$$

$\operatorname{Ret}^{2}(X, r)=\left([[X]], r_{[[X]]}\right)$, where $[[X]]=\left\{[a],[b],\left[x_{1}\right]\right\}, \mathcal{L}_{[[a]]}=\mathcal{L}_{[[b]]}=\mathcal{L}_{\left[\left[x_{1}\right]\right]}=e$

$\operatorname{Ret}^{3}(X, r)$ is the one element solution on $\{[a]\}$.

So we have $\operatorname{mpl}(X, r)=3$. We have seen that $\mathcal{G}$ is abelian, or equivalently, $\mathrm{sl} \mathcal{G}=1$. It is easy to see that $\operatorname{sl} G(X, r)=2$. Indeed, $\operatorname{mpl}(X, r)=3$ implies $2 \leq \operatorname{sl} G(X, r)$, by Theorem $7.23 \mathrm{sl} G(X, r) \leq \operatorname{sl} \mathcal{G}+1=2$.

Question 5.17. Are there multipermutation square-free solutions $(X, r)$ of arbitrarily high multipermutation level, and with abelian permutation group $\mathcal{G}$ ? If not, what is the largest integer $M$ for which there exist solutions $(X, r)$ with $\operatorname{mpl}(X, r)=$ $M$ and $\mathcal{G}$ abelian.

We show, see Theorem 6.1, that assuming $\mathcal{G}(X, r)$ abelian, one has $\operatorname{mpl}(X, r) \leq t$, where $t$ is the number of $\mathcal{G}$-orbits in $X$. We still do not have examples of solutions with high multipermutation level and $\mathcal{G}$ abelian.

\section{Solutions With ABELIAN PERMUtATION GRoup}

We can say a surprising amount about solutions $(X, r)$ for which $\mathcal{G}(X, r)$ is abelian. In this section we keep the notation and conventions from the previous sections.

As usual $(X, r)$ is a square-free solution of arbitrary cardinality, $\mathcal{G}=\mathcal{G}(X, r)$ denotes its YB permutation group. In the cases when we assume $X$ finite this will be written explicitly. Without restriction to the case of necessarily finite solutions we shall assume that $X$ has a finite number of $\mathcal{G}$ - orbits, as in the previous sections, $\mathcal{X}=\left\{X_{1}, \cdots, X_{t}\right\}$ will denote the set of $\mathcal{G}$-orbits in $X$. Clearly, in the case when $X$ is finite this condition is always in force. As discussed in the previous sections, each $\left(X_{i}, r_{i}\right), 1 \leq i \leq t$, is also a square-free solution, where $r_{i}$ is the restriction $r_{i}=r_{\mid X_{i}}$. The main results of the section are the following theorems. 
Theorem 6.1. Let $(X, r)$ be a square-free solution of arbitrary cardinality. Suppose its permutation group $\mathcal{G}$ is abelian, and $X$ has a finite number of $\mathcal{G}$-orbits, $X_{1}, \cdots, X_{t}$, where $t \geq 2$. Then the following conditions hold.

(1) Each $\left(X_{i}, r_{i}\right), 1 \leq i \leq t$, is a trivial solution.

(2) $(X, r)$ is a strong twisted union of its $\mathcal{G}$-orbits (see Definition [3.8):

$$
X=X_{1} \natural X_{2} \natural \cdots \curvearrowleft X_{t} .
$$

(3) $(X, r)$ is a multipermutation solution, with

$$
\operatorname{mpl}(X, r) \leq t .
$$

Corollary 6.2. Every finite square-free solution $(X, r)$ with abelian permutation group $\mathcal{G}$ has multipermutation level $\operatorname{mpl}(X, r) \leq t$, where $t$ is the number of its $\mathcal{G}$ orbits.

Theorem 6.3. Let $(X, r)$ be a square-free solution with abelian permutation group $\mathcal{G}$. Suppose $X$ is a strong twisted union, $(X, r)=X_{1} \natural X_{2}$ of the solutions $\left(X_{1}, r_{X_{1}}\right)$, and $\left(X_{2}, r_{X_{2}}\right)$. Then the three solutions are multipermutation solutions and

$$
\operatorname{mpl} X \leq \operatorname{mpl} X_{1}+\operatorname{mpl} X_{2} \text {. }
$$

Remark 6.4. Note that using a different argument Cedó, Jespers, and Okniński, CJR09, have proven that each finite square-free solution $(X, r)$ with $\mathcal{G}$ abelian is retractable (and therefore multipermutation solution), but do no give estimation of the multipermutation level.

Remark 6.5. Theorem 6.1 confirms Conjectures I and II GI in the case when the finite square-free solutions $(X, r)$ has abelian permutation group $\mathcal{G}(X, r)$, or equivalently its YB group $G(X, r)$ has solvable length 2 .

Remark 6.6. Note that in the general case there is no relation between $\mathrm{mpl} X$ and the number of orbits $t=t(X)$. As show Lemma 6.8, and Theorem 9.11, for every integer $m \geq 3$ there exist finite square-free solutions $(X, r)$ with exactly 2 orbits and with $\mathrm{mpl} X=m$.

The proofs of the main results in this section heavily rely on a necessary and sufficient condition for $\operatorname{mpl}(X, r)=m$ given by Theorem 7.10 in the next section. This condition is given in terms of long actions, or as we call them informally towers of actions. We need to develop first some basic technique for computation with towers of actions. We end the section with a simple construction doubling of solutions which is also used to illustrate that a solutions $(X, r)$ may have exactly two orbits and arbitrarily large finite multipermutation level $m=\operatorname{mpl} X$.

Definition 6.7. Let $\left(X, r_{X}\right)$ and $\left(X^{\prime}, r_{X^{\prime}}\right)$ be a disjoint identical finite square-free solution, where $X=\left\{x_{1} \cdots x_{n}\right\}, X^{\prime}=\left\{x_{1}^{\prime} \cdots x_{n}^{\prime}\right\}$. Let $Y=\{\alpha\}$ be a one element set disjoint with $X \bigcup X^{\prime}$, and let $\left(Y, r_{0}\right)$ be the one element trivial solution. (By definition one has $\operatorname{mpl} Y=0)$. Consider the extension of solutions $(Z, r)$

$$
Z=\left(X \natural_{0} X^{\prime}\right) \natural\{\alpha\},
$$

where $r$ is an extension of the YB maps, $r_{X}, r_{X^{\prime}}, r_{Y}$ defined (as usual) via the canonical isomorphism of solutions $\mathcal{L}_{\alpha}=\left(x_{1} x_{1}^{\prime}\right) \cdots\left(x_{n} x_{n}^{\prime}\right)$

$$
\begin{array}{r}
r_{\mid X}=r_{X}, \quad r_{\mid X^{\prime}}=r_{X^{\prime}} \\
r\left(x_{i}, x_{j}^{\prime}\right)=\left(x_{j}^{\prime}, x_{i}\right) \quad 1 \leq i, j \leq n \\
r\left(\alpha, x_{j}\right)=\left(x_{j}^{\prime}, \alpha\right), \quad r\left(\alpha, x_{j}^{\prime}\right)=\left(x_{j}, \alpha\right) .
\end{array}
$$


We call $(Z, r)$ a canonical doubling of $\left(X, r_{X}\right)$, and denote it $Z=X^{[2, \alpha]}$.

In the following lemma, wr denotes the wreath product of a group with a permutation group. We discuss wreath products more extensively below.

Lemma 6.8. Let $\left(X, r_{X}\right)$ be a square-free solution with $\operatorname{mpl} X=m$. Let $(Z, r)=$ $X^{[2, y]}$ be a canonical doubling of $\left(X, r_{X}\right)$. Denote $G_{X}=G\left(X, r_{X}\right), G_{Z}=G(Z, r)$. Then

(1) $\operatorname{mpl}(Z, r)=m+1$ and clearly, $(Z, r)$ has exactly two $\mathcal{G}(Z, r)$ orbits, namely $Z_{1}=X \cup X^{\prime}$, and $Z_{2}=\{y\}$.

(2) There is an isomorphism of groups

$$
G_{Z} \simeq\left(G_{X} \times G_{X}\right) \rtimes C_{\infty} \simeq G_{X} \text { wr } C_{\infty}
$$

where the generator of the infinite cyclic group interchanges the two factors.

(3) There is an isomorphism of groups

$$
\mathcal{G}(Z) \simeq\left(\mathcal{G}_{X} \times \mathcal{G}_{X}\right) \rtimes C_{2} \simeq \mathcal{G}_{X} \text { wr } C_{2} .
$$

The proof is straightforward.

Clearly, $\mathcal{L}_{\alpha} \in \operatorname{Aut}\left(X \natural_{0} X^{\prime}\right)$ but does not belong to the permutation group $\mathcal{G}\left(X \natural_{0}\right.$ $X^{\prime}$ ), so Lemma 6.8 is a particular case of Lemma 8.3 .

Remark 6.9. It is straightforward to see that

$$
\operatorname{sl}\left(G_{Z}\right)=\operatorname{sl}\left(G_{X}\right)+1, \quad \operatorname{sl}\left(\mathcal{G}_{Z}\right)=\operatorname{sl}\left(\mathcal{G}_{X}\right)+1,
$$

where $\operatorname{sl}(G)$ denotes the solvable length of the group $G$. We will see more general results later.

6.1. Computations with actions in $(X, r)$. In cases when we have to write a sequence of successive actions we shall use one also well known notation

$$
\alpha \triangleright x={ }^{\alpha} x
$$

Definition 6.10. Let $\zeta_{1}, \zeta_{2}, \cdots, \zeta_{m} \in X$. The expression

$$
\omega=\left(\cdots\left(\left(\zeta_{m} \triangleright \zeta_{m-1}\right) \triangleright \zeta_{m-2}\right) \triangleright \cdots \triangleright \zeta_{2}\right) \triangleright \zeta_{1}
$$

will be called a tower of actions or shortly a tower.

Clearly, the result of this action has the shape ${ }^{u} \zeta_{1}$, where

$$
u=\left(\cdots\left(\left(\zeta_{m} \triangleright \zeta_{m-1}\right) \triangleright \zeta_{m-2}\right) \triangleright \cdots \triangleright \zeta_{3}\right) \triangleright \zeta_{2},
$$

so it belongs to the $\mathcal{G}$-orbit of $\zeta_{1}$.

The following two remarks and lemma are straightforward and hold for the general case of square-free solutions, where $\mathcal{G}(X, r)$ is not necessarily abelian, and $X$ is of arbitrary cardinality.

Remark 6.11. Let $\Sigma_{1}, \Sigma_{2}$ be two disjoint alphabets, $m_{1}, m_{2}$ be positive integers, $m=m_{1}+m_{2}+1$. Let $\omega=\zeta_{m} \zeta_{m-1} \cdots \zeta_{2} \zeta_{1}$ be a string (word) of length $m$ in the alphabet $\Sigma_{1} \bigcup \Sigma_{2}$. Then one of the following conditions is satisfied

i) $\omega$ contains a segment $v$ of the shape $v=\beta y_{q} \cdots y_{2} y_{1} \alpha$, where $q \geq 1, y_{k} \in \Sigma_{j}, 1 \leq$ $k \leq q, \alpha, \beta \in \Sigma_{i}$, and $1 \leq i \neq j \leq 2$ 
ii) $\omega=y_{q} \cdots y_{2} y_{1} \alpha_{p} \cdots \alpha_{2} \alpha_{1}$, where $y_{s} \in \Sigma_{j}, 1 \leq s \leq q, \alpha_{k} \in \Sigma_{i}, 1 \leq k \leq p$, and either $p \geq m_{i}+1$, or $q \geq m_{j}+1,\left(p=0, q=m_{1}+m_{2}+1\right.$, or $p=m_{1}+m_{2}+1, q=0$ is also possible).

This remark has a transparent but very useful interpretation for towers of actions.

Remark 6.12. Let $X_{1}, X_{2}$ be disjoint subsets of the solution $(X, r), m_{1}, m_{2}$ be positive integers, $m=m_{1}+m_{2}+1$. Let $\zeta_{1}, \zeta_{2}, \cdots \zeta_{m} \in X_{1} \bigcup X_{2}$, and $\omega$ be the tower:

Then either

$$
\omega=\left(\cdots\left(\zeta_{m} \triangleright \zeta_{m-1}\right) \triangleright \cdots \triangleright \zeta_{2}\right) \triangleright \zeta_{1}
$$

i) $\omega$ contains a segment $\left(\left(\left(\cdots \triangleright \beta \triangleright y_{q}\right) \triangleright \cdots \triangleright y_{2}\right) \triangleright y_{1}\right) \triangleright \alpha$, where $q \geq 1, y_{k} \in X_{i}, 1 \leq k \leq$ $q, \alpha, \beta \in X_{j}$, and $1 \leq i \neq j \leq 2$; or

ii) $\omega$ has the shape

$$
\left.\omega=\left(\cdots\left(\left(\cdots\left(y_{q} \triangleright y_{q-1}\right) \triangleright \cdots \triangleright y_{2}\right) y_{1}\right) \triangleright \alpha_{p}\right) \cdots \alpha_{2}\right) \triangleright \alpha_{1},
$$

where $y_{s} \in X_{j}, 1 \leq s \leq q, \alpha_{k} \in X_{i}, 1 \leq k \leq p$, with $1 \leq i \neq j \leq 2$. Furthermore, either $p \geq m_{i}+1$, or $q \geq m_{j}+1,\left(p=0, q=m_{1}+m_{2}+1\right.$, or $q=m_{1}+m_{2}+1, q=0$ is also possible).

Lemma 6.13. Let $(X, r)$ be square-free solution, $y_{1}, \cdots y_{k} \in X$, with $k \geq 1$, and let $Z$ be an $r$-invarionat subset of $X$. Suppose there is an equality

$$
\left(\left(\cdots\left(\left(\alpha \triangleright y_{k}\right) \triangleright y_{k-1}\right) \triangleright \cdots y_{2}\right) \triangleright y_{1}\right)=\left(\cdots\left(y_{k} \triangleright y_{k-1}\right) \triangleright \cdots y_{2}\right) \triangleright y_{1} \quad \forall \alpha \in Z
$$

Then any longer tower

$\left.\left.\omega=\left(\cdots\left(\left((\cdots)\left(\left(\left(\left(\cdots\left(a_{s} \triangleright a_{s-1}\right) \triangleright \cdots\right) \triangleright a_{1}\right) \triangleright \alpha\right) \triangleright y_{k}\right) \triangleright y_{k-1}\right) \triangleright \cdots \triangleright y_{2}\right) \triangleright y_{1}\right) \triangleright b_{p}\right) \triangleright \cdots \triangleright b_{2}\right) \triangleright b_{1}$ with $a_{1}, \cdots a_{s}, b_{1}, \cdots b_{p} \in X$ and $\alpha \in Z$, can be simplified by "cutting" the leftmost sub-tower of length $s+1$, that is there is an equality:

$$
\omega=\omega^{\prime}=\left(\left(\cdots\left(\left(\left(\cdots\left(y_{k} \triangleright y_{k-1}\right) \triangleright \cdots \triangleright y_{2}\right) \triangleright y_{1}\right) \triangleright b_{p}\right) \triangleright \cdots \triangleright b_{2}\right) \triangleright b_{1} .\right.
$$

In the particular cases $s=0$ (respectively $p=0$ ) the a's, (respectively the b's) are simply missing in the formulae above.

The lemma follows straightforwardly from the clear implication

$$
\alpha \in Z \Longrightarrow\left(\left(\cdots\left(a_{s} \triangleright a_{s-1}\right) \triangleright \cdots\right) \triangleright a_{1}\right) \triangleright \alpha \in Z
$$

We shall refer (informally) to the procedure described in Lemma 6.13 as truncation.

From now on we assume that $\mathcal{G}(X, r)$ is abelian.

Lemma 6.14. Let $(X, r)$ be a square-free solution with abelian permutation group $\mathcal{G}$. Suppose $Y, Z$ are $r$-invariant subsets of $X$ and the following stu-type condition is satisfied:

$$
{ }^{\alpha} y={ }^{y} z \quad \forall \alpha, z \in Z \quad \text { and } \quad \forall y \in Y .
$$

Then for every pair $\alpha, z \in Z$, and every finite sequence $y_{1}, \cdots, y_{k} \in Y, k \geq 1$ one has

$$
\left.\left(\left(\cdots\left(\alpha \triangleright y_{k}\right) \triangleright y_{k-1}\right) \triangleright \cdots \triangleright y_{2}\right) \triangleright y_{1}\right) \triangleright z=\left(\left(\cdots\left(y_{k} \triangleright y_{k-1}\right) \triangleright \cdots \triangleright y_{2}\right) \triangleright y_{1}\right) \triangleright z .
$$


Proof. We shall prove (6.6) using induction on $k$. Clearly, 6.5 gives the base for the induction. Assume the statement of the lemma is true for $k-1$ where $k>1$. Suppose $\alpha, z \in Z, y_{1}, \cdots y_{k} \in Y$. For convenience we introduce the elements $y_{k-1}^{\prime}, y_{k-1}^{\prime \prime}$ as follows:

$$
y_{k-1}^{\prime}=y_{k} \triangleright y_{k-1}={ }^{y_{k}} y_{k-1} \quad y_{k-1}^{\prime \prime}=\left(\alpha \triangleright y_{k}\right) \triangleright y_{k-1}={ }^{\left({ }^{\alpha} y_{k}\right)} y_{k-1}
$$

Then the following equalities hold.

$$
\begin{aligned}
& \alpha \triangleright y_{k-1}^{\prime}={ }^{\alpha}\left(y_{k} y_{k-1}\right) \\
& ={ }^{\alpha} y_{k}\left(\alpha^{y_{k}} y_{k-1}\right) \quad \mathbf{1 1} \\
& ={ }^{y_{k}}\left({ }^{\alpha} y_{k} y_{k-1}\right) \quad \mathcal{G} \text { abelian } \\
& =\alpha^{y_{k}}\left(\left(\alpha \triangleright y_{k}\right) \triangleright y_{k-1}\right) \\
& =\left(\alpha^{y_{k}}\right) \triangleright y_{k-1}^{\prime \prime}
\end{aligned}
$$

Thus

$$
\alpha \triangleright y_{k-1}^{\prime}=\left(\alpha^{y_{k}}\right) \triangleright y_{k-1}^{\prime \prime} .
$$

Now consider the equalities

$$
\begin{array}{rlrl} 
& \left(\left(\cdots\left(\left(\alpha \triangleright y_{k}\right) \triangleright y_{k-1}\right) \triangleright \cdots y_{2}\right) \triangleright y_{1}\right) \triangleright z & \\
= & \left(\left(\cdots\left(y_{k-1}^{\prime \prime} \triangleright y_{k-2}\right) \triangleright \cdots \triangleright y_{2}\right) \triangleright y_{1}\right) \triangleright z & & \\
= & \left.\left(\left(\cdots\left(\left(\alpha^{y_{k}}\right) \triangleright y_{k-1}^{\prime \prime}\right) \triangleright y_{k-2}\right) \triangleright \cdots \triangleright y_{2}\right) \triangleright y_{1}\right) \triangleright z & & \alpha^{y_{k}} \in Z, \\
= & \left(\left(\cdots\left(\left(\alpha \triangleright y_{k-1}^{\prime}\right) \triangleright y_{k-2}\right) \triangleright \cdots \triangleright y_{2}\right) \triangleright y_{1}\right) \triangleright z & & \text { (6.8 }) \\
= & \left(\left(\cdots\left(y_{k-1}^{\prime} \triangleright y_{k-2}\right) \triangleright \cdots \triangleright y_{2}\right) \triangleright y_{1}\right) \triangleright z & & \text { by IH } \\
= & \left(\left(\cdots\left(\left(y_{k} \triangleright y_{k-1}\right) \triangleright y_{k-2}\right) \triangleright \cdots \triangleright y_{2}\right) \triangleright y_{1}\right) \triangleright z & & \text { (6.7) }
\end{array}
$$

where IH is the inductive assumption. This proves the Lemma.

Remark 6.15. Note that in the hypothesis of Lemma 6.14 we do not assume that the sets $Y, Z$ are disjoint. Furthermore the stu-type condition is not imposed symmetrically on both sets, i.e. even if $Y$ and $Z$ are disjoint we do not assume that necessarily $Y$ $Z$.

\subsection{Proofs of the theorems.}

Lemma 6.16. Let $(X, r)$ be a square-free solution, with abelian permutation group $\mathcal{G}$. Then the following two conditions hold.

(1) Let $Y$ be a $\mathcal{G}$-orbit of $X$. Then for any $x \in Y$ one has $\left(\mathcal{L}_{x}\right)_{\mid Y}=\operatorname{id}_{Y}$

(2) Suppose $Y, Z$ are two distinct $\mathcal{G}$-orbits of $X,\left(Y, r_{Y}\right),\left(Z, r_{Z}\right)$ are the canonically induced solutions on $Y$ and $Z$. Then the actions satisfy the stu condition:

$$
\left(\mathcal{L}_{x_{\alpha}}\right)_{\mid Y}=\left(\mathcal{L}_{\alpha}\right)_{\mid Y} ; \quad\left(\mathcal{L}_{\alpha}\right)_{\mid Z}=\left(\mathcal{L}_{x}\right)_{\mid Z} \quad \text { for all } \quad x \in Y, \alpha \in Z
$$

Moreover, the induced solution $\left(T, r_{T}\right)$ on the union $T=Y \bigcup Z$ is a strong twisted union $T=Y \boxminus Z$.

Proof. Let $x \in Y$. To prove (1) it will be enough to show

$$
{ }^{x}\left({ }^{t} x\right)={ }^{t} x, \forall t \in X \text {. }
$$

Now the equalities

$$
\begin{aligned}
{ }^{x}\left({ }^{t} x\right) & ={ }^{t}\left({ }^{x} x\right) & & \mathcal{G} \text { abelian } \\
& ={ }^{t} x & & (X, r) \text { square-free } .
\end{aligned}
$$


imply (1). Assume now that $x, y \in Y, \alpha, \beta \in Z$. We have to show

$$
{ }^{y} \alpha={ }^{\alpha} x, \quad{ }^{\beta} x \alpha={ }^{x} \alpha .
$$

Consider the equalities

$$
\begin{aligned}
{ }^{\alpha} x & ={ }^{y}\left({ }^{\alpha} x\right) & & y,{ }^{\alpha} x \in Y \text { and }(1) \\
& ={ }^{\left({ }^{\alpha} \alpha\right)}\left(y^{\alpha} x\right) & & \text { by } \mathbf{1 1} \\
& { }^{\left({ }^{y} \alpha\right)} x & & x, y^{\alpha} \in Y \text { and }(1)
\end{aligned}
$$

We have shown the left hand side equality in (6.10). Analogous argument gives the remaining equality.

Corollary 6.17. Let $(X, r)$ be a square-free solution of arbitrary cardinality and with abelian permutation group $\mathcal{G}$. Suppose $\mathcal{G}$ acts non transitively on $X$ and splits it into a finite number of $\mathcal{G}$ - orbits $X_{1}, \cdots X_{t}, t \geq 2$. Then each $\left(X_{i}, r_{i}\right)$ is a trivial solution and $X$ is a strong twisted union

$$
X=X_{1} \natural X_{2} \natural \cdots \natural X_{t} .
$$

Proposition 6.18. Under the hypothesis of Theorem 6.1. $(X, r)$ is multipermutation solution with $\operatorname{mpl} X \leq t$, where $t$ is the number of $\mathcal{G}$-orbits of $X$.

Proof. By Theorem7.10 it will be enough to show that for each choice of $y_{1}, \cdots y_{m+1} \in$ $X$ there is an equality

$$
\omega:=\left(\cdots\left(\left(y_{m+1} \triangleright y_{m}\right) \triangleright y_{m-1}\right) \triangleright \cdots \triangleright y_{2}\right) \triangleright y_{1}=\left(\cdots\left(y_{m} \triangleright y_{m-1}\right) \triangleright \cdots \triangleright y_{2}\right) \triangleright y_{1}=: \omega^{\prime} .
$$

Clearly, since the orbits are exactly $m$, there will be some $1 \leq \lambda<\lambda+\mu \leq m+1$, such that $y_{\lambda}, y_{\lambda+\mu}$ are in the same orbit, say $X_{i}$.

Two cases are possible.

Case 1. $\mu=1$. In this case, $\left.\lambda+\mu=\lambda+1\left(y_{m+1} \triangleright y_{m}\right) \triangleright \cdots \triangleright y_{\lambda+1}\right)=u \in X_{i}$ and since $X_{i}$ is a trivial solution, one has $\left(\cdots\left(y_{m+1} \triangleright y_{m}\right) \triangleright \cdots \triangleright y_{\lambda+1}\right) \triangleright y_{\lambda}={ }^{u} y_{\lambda}=y_{\lambda}$, thus

$$
\omega=\left(\cdots\left(\left(y_{m+1} \triangleright y_{m}\right) \triangleright y_{m-1}\right) \triangleright \cdots \triangleright y_{2}\right) \triangleright y_{1}=\left(\cdots\left(y_{\lambda} \triangleright y_{\lambda-1}\right) \triangleright \cdots \triangleright y_{2}\right) \triangleright y_{1}
$$

Similarly,

$$
\omega^{\prime}=\left(\cdots\left(y_{m} \triangleright y_{m-1}\right) \triangleright \cdots \triangleright y_{2}\right) \triangleright y_{1}=\left(\cdots\left(y_{\lambda} \triangleright y_{\lambda-1}\right) \triangleright \cdots \triangleright y_{2}\right) \triangleright y_{1}
$$

So $\omega=\omega^{\prime}$, which proves (6.11).

Case 2. $\mu>1$. In this case we set $y_{\lambda+\mu}=\alpha, y_{\lambda}=z$. The tower $\omega$ contains the segment $\left(\cdots\left(\alpha \triangleright y_{\lambda+\mu-1}\right) \triangleright \cdots \triangleright y_{\lambda+1}\right) \triangleright z$, with $\alpha, z \in X_{i}$. By Lemma 6.14 we can cut $\alpha$ from the tower to yield

$$
\left(\cdots\left(\alpha \triangleright y_{\lambda+\mu-1}\right) \triangleright \cdots \triangleright y_{\lambda+1}\right) \triangleright z=\left(\cdots\left(y_{\lambda+\mu-1} \triangleright y_{\lambda+\mu-2}\right) \cdots \triangleright y_{\lambda+1}\right) \triangleright z .
$$

We shall assume $\lambda+\mu<m+1$ (The proof in the case $\lambda+\mu=m+1$ is analogous). By Lemma 6.13 there are equalities

$$
\begin{aligned}
\omega & =\left(\cdots\left(\left(\cdots\left(\left(\cdots\left(y_{m+1} \triangleright y_{m}\right) \triangleright \cdots \alpha\right) \triangleright y_{\lambda+\mu-1}\right) \triangleright \cdots \triangleright y_{\lambda+1}\right) \triangleright z\right) \triangleright \cdots\right) \triangleright y_{1} \\
& =\left(\cdots\left(\left(\cdots\left(y_{\lambda+\mu-1} \triangleright y_{\lambda+\mu-2}\right) \cdots \triangleright y_{\lambda+1}\right) \triangleright z\right) \triangleright \cdots\right) \triangleright y_{1} \\
& \left.=\left(\cdots\left(\left(\cdots\left(\cdots\left(y_{m} \triangleright y_{m-1}\right) \triangleright \cdots \alpha\right) \triangleright y_{\lambda+\mu-1}\right) \triangleright \cdots \triangleright y_{\lambda+1}\right) \triangleright z\right) \triangleright \cdots\right) \triangleright y_{1} .
\end{aligned}
$$

The proposition has been proved. 
Theorem 6.1 follows straightforwardly from Corollary 6.17 and Proposition 6.18

We shall now prove Theorem 6.3. Suppose the square free solution $(X, r)$ is a strong twisted union $(X, r)=X_{1} \curvearrowleft X_{2}$ and $\mathcal{G}(X, r)$ is abelian. Then by Theorem $6.1(X, r),\left(X_{1}, r_{X_{1}}\right),\left(X_{2}, r_{X_{2}}\right)$ are multipermutation solutions. Let $\mathrm{mpl} X_{1}=m_{1}$, $\operatorname{mpl} X_{2}=m_{2}$. We claim that $\operatorname{mpl} X \leq m_{1}+m_{2}$. Denote $m=m_{1}+m_{2}+1$. By Theorem 7.10 it will be enough to show that for any choice of $\zeta_{1}, \cdots \zeta_{m} \in X$ there is an equality

$$
\omega=\left(\cdots\left(\zeta_{m} \triangleright \zeta_{m-1}\right) \triangleright \cdots \triangleright \zeta_{2}\right) \triangleright \zeta_{1}=\omega^{\prime}=\left(\cdots\left(\zeta_{m-1} \triangleright \zeta_{m-2}\right) \triangleright \cdots \triangleright \zeta_{2}\right) \triangleright \zeta_{1}
$$

By Remark 6.12 two cases are possible.

Case 1. $\omega$ contains a segment $\left(\left(\left(\cdots \triangleright \beta \triangleright y_{q}\right) \triangleright \cdots \triangleright y_{2}\right) \triangleright y_{1}\right) \triangleright \alpha$, where $q \geq 1, y_{k} \in$ $X_{i}, 1 \leq k \leq q, \alpha, \beta \in X_{j}$, and $1 \leq i \neq j \leq 2$. Since $X$ is strong twisted union of $X_{1}$ and $X_{2}$ the hypothesis of Lemma 6.14 is in force, and therefore

$$
\left(\left(\cdots\left(\beta \triangleright y_{q}\right) \triangleright \cdots \triangleright y_{2}\right) \triangleright y_{1}\right) \triangleright \alpha=\left(\left(\cdots\left(y_{q} \triangleright y_{q-1}\right) \triangleright \cdots \triangleright y_{2}\right) \triangleright y_{1}\right) \triangleright \alpha
$$

Now apply Lemma 6.13 to deduce (6.12)

Case 2. $\omega$ has the shape

$$
\omega=\left(\cdots\left(\left(\left(\cdots\left(y_{q} \triangleright y_{q-1}\right) \triangleright \cdots \triangleright y_{2}\right) \triangleright y_{1}\right) \triangleright \alpha_{p}\right) \cdots \alpha_{2}\right) \triangleright \alpha_{1},
$$

where $y_{s} \in X_{j}, 1 \leq s \leq q, \alpha_{k} \in X_{i}, 1 \leq k \leq p$, with $1 \leq i \neq j \leq 2$. Furthermore, either $p \geq m_{i}+1$, or $q \geq m_{j}+1,\left(p=0, q=m_{1}+m_{2}+1\right.$, or $q=m_{1}+m_{2}+1, q=0$ is also possible). Without loss of generality we may assume $q \geq m_{j}+1$. But $\operatorname{mpl} X_{j}=m_{j}$, so Theorem 7.10 implies the equality

$$
\left(\cdots\left(y_{q} \triangleright y_{q-1}\right) \triangleright \cdots \triangleright y_{2}\right) \triangleright y_{1}=\left(\cdots\left(y_{q-1} \triangleright y_{q-2}\right) \triangleright \cdots \triangleright y_{2}\right) \triangleright y_{1} .
$$

We apply again Lemma 6.13 to obtain (6.12). The case when $p \geq m_{i}+1$ is analogous and we leave it to the reader.

Theorem 6.3 has been proved. Q.E.D.

\section{Multipermutation solutions of Finite multipermutation LeVel}

7.1. General results. We shall use the notation from section 2. We also recall some notions and basic facts from GI].

Notation 7.1. Let $(X, r)$ be a square-free solution. For each integer $k \geq 0$ as usual, we shall use following notation.

(1) $\operatorname{Ret}^{k}(X, r)$ denotes the $k$-th retract of $(X, r)$, but when $k=1$ it is convenient to use both notations $\operatorname{Ret}(X, r)=\operatorname{Ret}^{1}(X, r)$ and $\left([X], r_{[X]}\right)$ for the retract. For completeness we set $\operatorname{Ret}^{0}(X, r)=(X, r)$.

(2) $x^{(k)}$ denotes the image of $x$ in $\operatorname{Ret}^{k}(X, r)$. The set

$$
\left[x^{(k)}\right]:=\left\{\xi \in X \mid x^{(k)}=\xi^{(k)}\right\}
$$

is called the kth retract class of $x$. (In [GI] it is referred to as the kth retract orbit of $x$ ).

(3) In the case when $\operatorname{mpl}(X, r)=m<\infty$, and $X$ has a finite number of $\mathcal{G}$-orbits, we let these orbits be

$$
X_{1}, \ldots, X_{t} .
$$


(4) We fix a notation for the elements of the $(m-1)$ th retract:

$$
\stackrel{m-1}{\operatorname{Ret}}(X, r)=\left\{\zeta_{1}^{(m-1)}, \cdots, \zeta_{s}^{(m-1)}\right\} .
$$

(By Corollary 7.9 one has $s \leq t$ ).

(5) The $m-1$ th retract classes will be denoted by

$$
Y_{i}:=\left[\zeta_{i}^{(m-1)}\right], 1 \leq i \leq s .
$$

For each $i, 1 \leq i \leq s$ we denote the set of all $\mathcal{G}$-orbits of $X$ which intersect $Y_{i}$ nontrivially by

$$
X_{i 1}, X_{i 2}, \cdots, X_{i t_{i}} .
$$

Remark 7.2. In the above notation, suppose that $\operatorname{mpl}(X, r)=m<\infty$, and that $X$ has a finite number of $\mathcal{G}$-orbits, say $X_{1}, \ldots, X_{t}$. Then by Corollary $7.9 \operatorname{Ret}^{m-1}$ is a finite set of order $s, 2 \leq s \leq t$.

Furthermore, it follows from Proposition 7.8 that for each pair $i, j 1 \leq i \leq s, 1 \leq$ $j \leq t$ one has

$$
Y_{i} \bigcap X_{j} \neq \emptyset \Longleftrightarrow X_{j} \subseteq Y_{i} .
$$

so each $(m-1)$ th retract class $Y_{i}, 1 \leq i \leq s$ is a disjoint union of the set of all $\mathcal{G}$-orbits which intersect it nontrivially:

$$
Y_{i}=\bigcup_{1 \leq k \leq t_{i}} X_{i k}
$$

The following corollary is straightforward from Lemma 2.24

Corollary 7.3. For each integer $k \geq 1$ the canonical map $(X, r) \longrightarrow \operatorname{Ret}^{k}(X, r)$, $x \mapsto x^{(k)}$, is a homomorphism of solutions.

The following results are extracted from [GI], where they are stated for finite squarefree solutions $(X, r)$. However, the argument does not rely on the finiteness of $X$.

Facts 7.4. GI]

(1) [GI], Lemma 8.10. For every $\alpha, \beta, x \in X$, and $k$ a positive integer,

$$
\alpha^{(k)}=\beta^{(k)} \Longrightarrow\left({ }^{\alpha} x\right)^{(k-1)}=\left({ }^{\beta} x\right)^{(k-1)} .
$$

In particular,

$$
\alpha^{(2)}=\beta^{(2)} \Longrightarrow{ }^{\alpha} x \sim{ }^{\beta} x \quad \forall x \in X, \quad{ }^{\alpha} \beta \sim \beta .
$$

(2) GI], Lemma 8.9. It follows (7.1) that for any positive integer $k$, and any $x \in X$, the restriction $r_{x, k}$ of $r$ on $\left[x^{(k)}\right]$ is a bijective map

$$
r_{x, k}:\left[x^{(k)}\right] \times\left[x^{(k)}\right] \longrightarrow\left[x^{(k)}\right] \times\left[x^{(k)}\right],
$$

so the kth retract class $\left(\left[x^{(k)}\right], r_{x, k}\right)$ is itself a solution. Furthermore, $\left(\left[x^{(k)}\right], r_{x, k}\right)$ is a multipermutation solution of level $\leq k$. In particular, whenever $[x]$ has cardinality $\geq 2,\left([x], r_{x, 1}\right)$ is the trivial solution.

(3) GI], Lemma 8.12.

$$
\alpha^{(2)}=\beta^{(2)} \Longrightarrow{ }^{\alpha} x={ }^{x} \beta \quad \forall x \in X, \quad\left(\mathcal{L}_{\alpha x}\right)_{\mid\left[\alpha^{(2)}\right]}=\left(\mathcal{L}_{x}\right)_{\mid\left[\alpha^{(2)}\right]} .
$$

Remark 7.5. Note that Lemma 8.9 in [GI] states inaccurately that $\operatorname{mpl}\left(\left[x^{(k)}\right], r_{x, k}\right)=$ $k$. The correct statement is $\operatorname{mpl}\left(\left[x^{(k)}\right], r_{x, k}\right) \leq k$. 
Remark 7.6. Suppose that $X_{0}=X \bigcap \operatorname{ker} \mathcal{L} \neq \emptyset$ then $\mathcal{G}\left(X_{0}\right)=1$. Let $\zeta_{0} \in X_{0}$. Then $X_{0}=\left[\zeta_{0}\right] \subseteq\left[\zeta_{0}^{(k)}\right]$, for all $k \geq 1$. Let $Y=\left[x^{(k)}\right]$ be a $k$-th retract class distinct from $\left[\zeta_{0}^{(k)}\right]$. Then this class generates a nontrivial permutation group $\mathcal{G}(Y)$. It follows then that $\mathcal{G}(Y)=1$ is possible for at most one k-th retract class, namely $Y=\left[\zeta_{0}^{(k)}\right]$, and this happens in the particular case $Y=\left[\zeta_{0}^{(k)}\right]=X_{0}$.

Corollary 7.7. Suppose $Y=\left[\zeta^{(2)}\right]$ is a second retract class in $X$. Then the permutation group $\mathcal{G}(Y)$ is an abelian subgroup of $\mathcal{G}=\mathcal{G}(X, r)$. $\mathcal{G}(Y)=1$ iff $Y \subset \operatorname{ker} \mathcal{L}$.

Proof. Let $\alpha, \beta \in Y$. Then $\alpha^{(2)}=\beta^{(2)}$, and by (7.2) one has

$$
{ }^{\alpha} \beta \sim{ }^{\beta} \beta=\beta \text {, }
$$

or equivalently

$$
\mathcal{L}_{(\alpha \beta)}=\mathcal{L}_{\beta}
$$

similarly

$$
\mathcal{L}_{\alpha^{\beta}}=\mathcal{L}_{\alpha}
$$

So we have

$$
\begin{aligned}
\mathcal{L}_{\alpha} \circ \mathcal{L}_{\beta} & =\mathcal{L}_{\alpha \beta} \circ \mathcal{L}_{\alpha^{\beta}} & & \text { lri } \\
& =\mathcal{L}_{\beta} \circ \mathcal{L}_{\alpha} & & (7.4),(7.5),
\end{aligned}
$$

hence $\mathcal{G}(Y)$ is abelian.

Proposition 7.8. The following conditions are equivalent:

(1) $\operatorname{mpl}(X, r)=m$.

(2) For every $x \in X$ one has

$$
X \supset\left[x^{(m-1)}\right] \supseteq \mathcal{O}_{\mathcal{G}}(x),
$$

where the left hand side inclusion is strict, and $\mathcal{O}_{\mathcal{G}}(x)$ is the $\mathcal{G}$-orbit of $x$.

(3) For every $x \in X$ the $m-1$ retract class $\left[x^{(m-1)}\right]$ is a $\mathcal{G}$-invariant proper subset of $X$.

Proof. Note that $\operatorname{mpl}(X, r)=m$ iff $\operatorname{Ret}^{m-1}$ is a trivial solution with at least 2 elements. Clearly, $\operatorname{Ret}^{m-1}$ is a trivial solution iff

$$
{ }^{\left(a^{(m-1)}\right)}\left(x^{(m-1)}\right)=x^{(m-1)} \quad \forall a, x \in X .
$$

The following are equalities in $\operatorname{Ret}^{m-1}$

$$
\begin{aligned}
&{ }^{\left(a^{(m-1)}\right)}\left(x^{(m-1)}\right)=\left({ }^{a} x\right)^{(m-1)} \quad \forall a, x \in X \\
& \operatorname{mpl}(X, r)=m \Longleftrightarrow \operatorname{Ret}^{m-1} \quad \text { is a trivial solution of order } \geq 2 \\
& \Longleftrightarrow\left({ }^{a} x\right)^{(m-1)}=x^{(m-1)} \quad \forall a, x \in X,\left[x^{(m-1)}\right] \subset X \\
& \Longleftrightarrow \mathcal{O}_{\mathcal{G}}(x) \subseteq\left[x^{(m-1)}\right] \subset X \quad \forall a, x \in X \\
& \Longleftrightarrow\left[x^{(m-1)}\right] \quad \text { is a } \mathcal{G} \text {-invariant proper subset of } X .
\end{aligned}
$$

Corollary 7.9. Let $(X, r)$ be a square-free multipermutation solution of arbitrary cardinality. Suppose it is a multipermutation solution with $2 \leq m p l X=m<$ $\infty$. Then the number of $\mathcal{G}$ - orbits in $X$ is at least the cardinality of $\operatorname{Ret}^{m-1}$. In particular, $\mathcal{G}$ acts intransitively on $X$. 
Proof. The (m-1)st retract $\operatorname{Ret}^{m-1}$ is a trivial solution with at least 2 elements. By Proposition 7.8 each $(m-1)$-retract class $\left[x^{(m-1)}\right]$ contains the $\mathcal{G}$-orbit of $x$. This proves the corollary.

Theorem 7.10. Let $(X, r)$ be an arbitrary square-free solution, not necessarily of finite cardinality. Then

i) $\operatorname{mpl}(X, r) \leq m$ if and only if the following equality holds

$$
\begin{gathered}
\left(\left(\cdots\left(\left(y_{m} \triangleright y_{m-1}\right) \triangleright y_{m-2}\right) \triangleright \cdots \triangleright y_{2}\right) \triangleright y_{1}\right) \triangleright x \\
\forall x, y_{1}, \cdots y_{m} \in X .
\end{gathered}=\left(\left(\cdots\left(y_{m-1} \triangleright y_{m-2}\right) \triangleright \cdots \triangleright y_{2}\right) \triangleright y_{1}\right) \triangleright x,
$$

ii) $\operatorname{mpl}(X, r)=m$ if and only if $m$ is the minimal integer for which 7.6) holds.

Proof. We use induction on $m$ to show the implication

$$
\operatorname{mpl} X \leq m \Longleftrightarrow(7.6) \text {. }
$$

Clearly,

$$
\left.\operatorname{mpl} X \leq 2 \Longleftrightarrow\left[{ }^{z} y\right]=[y], \forall y, z \in X \Longleftrightarrow{ }^{(z} y\right) x={ }^{y} x, \forall x, y, z \in X .
$$

This gives the base for the induction.

Assume the implications are true whenever $\operatorname{mpl} X \leq m$. Consider now the retract $\left([X], r_{[X]}\right.$. Clearly $\mathrm{mpl} X=\operatorname{mpl}[X]+1$. Furthermore, by the inductive assumption the following equality holds if and only if $\left.\operatorname{mpl}\left([X], r_{[} X\right]\right) \leq m$.

$\left(\left(\cdots\left(\left(\left[y_{m+1}\right] \triangleright\left[y_{m}\right]\right) \triangleright\left[y_{m-1}\right]\right) \triangleright \cdots\left[y_{3}\right]\right) \triangleright\left[y_{2}\right]\right) \triangleright\left[y_{1}\right]=\left(\cdots\left(\left(\left[y_{m}\right] \triangleright\left[y_{m-1}\right]\right) \triangleright \cdots\left[y_{3}\right]\right) \triangleright\left[y_{2}\right]\right) \triangleright\left[y_{1}\right]$

for all $y_{1}, \cdots, y_{m}, y_{m+1} \in X$.

(Here we enumerate differently: we write $y_{1}$ instead of $x$, etc.) By the obvious equalities

$$
[a] \triangleright[b]={ }^{[a]}[b]=\left[{ }^{a} b\right]=[a \triangleright b],
$$

(7.7) is equivalent to

$$
\left[\left(\cdots\left(\left(y_{m+1} \triangleright y_{m}\right) \triangleright y_{m-1}\right) \triangleright \cdots \triangleright y_{2}\right) \triangleright y_{1}\right]=\left[\left(\cdots\left(y_{m} \triangleright y_{m-1}\right) \triangleright \cdots \triangleright y_{2}\right) \triangleright y_{1}\right]
$$

for all $y_{1}, \cdots, y_{m}, y_{m+1} \in X$.

But (7.9) is equivalent to

$$
\left(\left(\cdots\left(\left(y_{m+1} \triangleright y_{m}\right) \triangleright y_{m-1}\right) \triangleright \cdots \triangleright y_{2}\right) \triangleright y_{1}\right) \triangleright x=\left(\cdots\left(\left(y_{m} \triangleright y_{m-1}\right) \triangleright \cdots \triangleright y_{2}\right) \triangleright y_{1}\right) \triangleright x
$$

for all $x, y_{1}, \cdots y_{m}, y_{m+1} \in X$.

This proves the equivalence mpl $X \leq m+1 \Longleftrightarrow 7.10$, which proves i).

(ii) follows straightforwardly from (i).

The theorem has been proved. 
7.2. The groups $G(X, r)$ and $\mathcal{G}(X, r)$. We recall more results.

Facts 7.11. Suppose $(X, r)$ is a square-free solution. Then

(1) $G=G(X, r)$ is torsion free. Let $p$ be the least common multiple of the orders of all permutations $\mathcal{L}_{x}$ for $x \in X$. Then the following conditions hold.

(i) $y x^{p}=\left(\left({ }^{y} x\right)^{p}\right) y, \quad x^{p} y=y\left(x^{y}\right)^{p} \quad \forall x, y \in X$. Thus the group $G$ acts via conjugation on the set $X^{(p)}=\left\{x^{p} \mid x \in X\right\}$.

(ii) $x^{p} y^{p}=y^{p} x^{p}, \forall x, y \in X$. The subgroup $A$ of $G$ generated by the set $X^{(p)}=\left\{x^{p} \mid x \in X\right\}$ is isomorphic to the free abelian group in $n$ generators, (see GI, GB]).

(2) It is known that each set-theoretic solution (X, $r$ ) of YBE (a braded set) can be extended canonically to a solution $\left(S, r_{S}\right)$ on $S=S(X, r)$, see GIM08 (respectively to a solution $\left(G, r_{G}\right)$, on $G=G(X, r)$, , [YZ) which makes $\left(S, r_{S}\right), a$ braided monoid (respectively $\left(G, r_{G}\right), a$ braided group. In other words the equality

$$
u v={ }^{u} v \cdot u^{v},
$$

holds in for all $u, v$ in $S$ (respectively in $G$ ).

Remark 7.12. It follows from the results of GIM08, that the extended solution $\left(G, r_{G}\right)$ satisfy

(1) $\left(G, r_{G}\right)$ is involutive (i.e. $\left(G, r_{G}\right)$ is symmetric set) if and only if $(X, r)$ is involutive.

(2) $\left(G, r_{G}\right)$ is nondegenerate if and only if $(X, r)$ is nondegenerate

(3) In particular, if $(X, r)$ is a square-free solution then $\left(G, r_{G}\right)$ is a nondegenerate symmetric set (but in general it is not square-free). The notion of equivalence $u \sim v$ given by

$$
u \sim v \Leftrightarrow(\forall g \in G)\left({ }^{u} g={ }^{v} g\right)
$$

is well defined, and, as usual, [u] denotes the equivalence class of $u$ in $G$.

Lemma 7.13. Let $G=G(X, r)$. The kernel $K_{0}=\operatorname{ker} \mathcal{L}$ of the group homomorphism $\mathcal{L}: G \longrightarrow \operatorname{Sym}(X)$ is a normal abelian subgroup of $G$ of finite index. In particular, $K_{0}$ contains the free abelian subgroup $A={ }_{g r}\left[x_{1}^{p}, \cdots, x_{n}^{p}\right]$, where $p$ is the least common multiple of all orders of permutations $\mathcal{L}_{x}$, for $x \in X$.

Proof. Clearly $u \in K_{0}$ if and only if $\mathcal{L}_{u}=\mathrm{id}_{X}$, and by lri the right action $\mathcal{R}_{u}=$ $\left(\mathcal{L}_{u}\right)^{-1}=\mathrm{id}_{X}$. This straightforwardly implies

$$
u \in K_{0} \Longleftrightarrow{ }^{u} a=a \quad \forall a \in G \Longleftrightarrow a^{u}=a, \quad \forall a \in G
$$

Assume now $u, v \in K_{0}$ Then $u v=7.11$ v $u \cdot u^{v}=\sqrt{7.12} v u$, so $K_{0}$ is abelian. Clearly, $\mathcal{L}_{x^{p}}=\left(\mathcal{L}_{x}\right)^{p}=\mathrm{id}_{X}$, so $x^{p} \in K_{0}$, for all $x \in X$, and therefore the free abelian group $A$ is contained in $K_{0}$.

In assumption and conventions as above we introduce more notation.

Notation 7.14. (1) $G_{i}=G\left(\operatorname{Ret}^{i}(X, r)\right), G_{0}=G(X, r)=G\left(\operatorname{Ret}^{0}(X, r)\right):=$ $(X, r))$.

(2) $\mathcal{G}_{i}=\mathcal{G}\left(\operatorname{Ret}^{i}(X, r)\right)$. 
(3) $\mathcal{L}^{0}=\mathcal{L}: G(X, r) \longrightarrow \mathcal{G}(X, r)$ is the usual epimorphism extending the assignment $x \mapsto \mathcal{L}_{x}, x \in X$

$\mathcal{L}^{i}=\mathcal{L}: G_{i} \longrightarrow \mathcal{G}_{i}$ is the canonical epimorphism extending the assignment $x^{(i)} \mapsto \mathcal{L}_{x^{(i)}} \in \operatorname{Sym}\left(\operatorname{Ret}^{i}(X, r)\right), x \in X$.

(4) $K_{i}$, is the pull-back of $\operatorname{ker} \mathcal{L}^{i}$ in $G$, in particular $K_{0}=\operatorname{ker} \mathcal{L}$.

(5) $\mu_{i}: G_{i} \longrightarrow G_{i+1}$ are the canonical epimorphisms extending $x^{(i)} \mapsto x^{(i+1)}$, where $0 \leq i<\operatorname{mpl} X$, see Lemma 7.15 and Proposition 7.17.

(6) $N_{i}$ is the pull-back of $\operatorname{ker} \mu_{i}$ in $G$.

(7) $\varphi_{i}: \mathcal{G}_{i} \longrightarrow \mathcal{G}_{i+1}$ is the canonical epimorphism extending the assignments $\mathcal{L}_{\left(x^{(i)}\right)} \mapsto \mathcal{L}_{x^{(i+1)}}, x \in X$, see Lemma 7.15 and Proposition 7.17

(8) $H_{i}$ is the pull-back of $\operatorname{ker} \varphi_{i}$ in $G$.

Note that by definition, for $1 \leq i \leq m-1$ one has

$$
K_{1}=\left\{u \in S \mid \mathcal{L}_{[u]}=\operatorname{id}_{[X]}\right\}, \quad K_{i}=\left\{u \in S \mid \mathcal{L}_{\left(u^{(i)}\right)}=\operatorname{id}_{\operatorname{Ret}^{i}(X, r)}\right\} .
$$

Lemma 7.15. In assumption and notation as above the following conditions hold.

(1) The canonical epimorphism of solutions

$$
\mu:(X, r) \longrightarrow([X],[r]) ; \quad x \mapsto[x],
$$

extends to a group epimorphism $\mu_{0}: G_{0} \longrightarrow G_{1}$. Analogously there exists a group epimorphism $\mu_{1}: G_{1} \longrightarrow G_{2}$.

(2) There is a canonical epimorphism $\varphi_{0}: \mathcal{G}_{0} \longrightarrow \mathcal{G}_{1} \quad \mathcal{L}_{x} \mapsto \mathcal{L}_{[x]}, \forall x \in X$.

(3) The kernels $N_{0}=\operatorname{ker} \mu_{0}, K_{0}=\operatorname{ker} \mathcal{L}^{0}, H_{0}$ - the pull back of $\operatorname{ker} \varphi_{0}$ into $G$, and $K_{1}$, the pull back of $\operatorname{ker} \mathcal{L}^{1}$ into $G$ satisfy

$$
\begin{gathered}
N_{0} \subset K_{0} \subset K_{1}=H_{0} \\
\operatorname{ker} \mu_{1} \simeq N_{1} / N_{0} ; \quad \operatorname{ker} \mathcal{L}_{1} \simeq K_{1} / N_{0} ; \quad \operatorname{ker} \varphi_{0} \simeq K_{1} / K_{0} .
\end{gathered}
$$

(4) In particular, $N_{0}$ is an abelian normal subgroup of $G_{0}$, and there is a canonical epimorphism of groups

$$
f_{0}: G_{1} \longrightarrow \mathcal{G}_{0} \quad[x] \mapsto \mathcal{L}_{[x]}, \quad x \in X \quad \text { with } \operatorname{ker} f_{0} \simeq K_{0} / N_{0}
$$

(5) There are short exact sequences:

$$
\begin{array}{cc}
1 \longrightarrow N_{0} \longrightarrow G \stackrel{\mu_{0}}{\longrightarrow} G_{1} \longrightarrow 1 & 1 \longrightarrow K_{0} \longrightarrow G \stackrel{\mathcal{L}^{0}}{\longrightarrow} \mathcal{G} \longrightarrow 1 \\
1 \longrightarrow N_{1} / N_{0} \longrightarrow G_{1} \stackrel{\mu_{1}}{\longrightarrow} G_{2} \longrightarrow 1 & 1 \longrightarrow K_{0} / N_{0} \longrightarrow G_{1} \stackrel{f_{0}}{\longrightarrow} \mathcal{G} \longrightarrow 1 \\
1 \longrightarrow K_{1} / N_{0} \longrightarrow G_{1} \stackrel{\mathcal{L}^{1}}{\longrightarrow} \mathcal{G}_{1} \longrightarrow 1 & 1 \longrightarrow K_{1} / K_{0} \longrightarrow \mathcal{G} \stackrel{\varphi_{0}}{\longrightarrow} \mathcal{G}_{1} \longrightarrow 1,
\end{array}
$$

Moreover, the following diagram is commutative: 


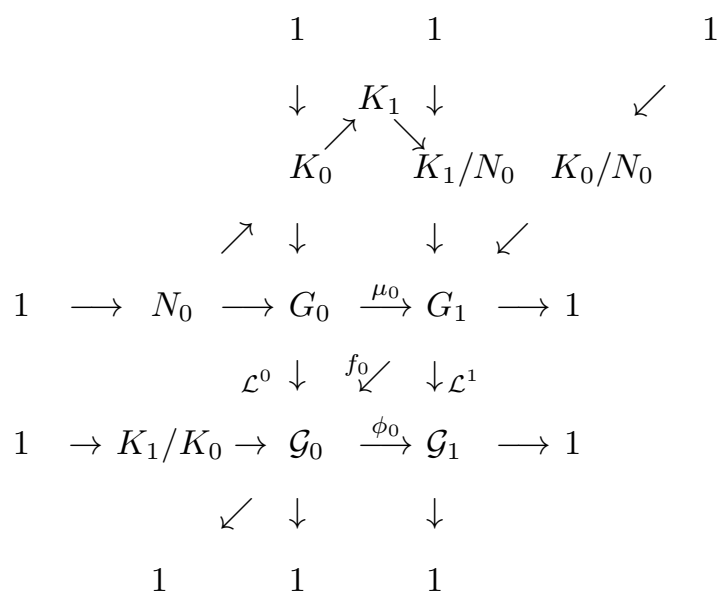

Proof. Parts (1), (2) are clear. We shall verify (3). Clearly, the kernel $N_{0}=\operatorname{Ker} \mu_{G}$ consists of all $a \in G$, such that $[a]=1_{[G]}$, hence it will be enough to show the implication

$$
[a]=1_{[G]} \Longrightarrow \mathcal{L}_{a}=\mathrm{id}_{X}
$$

Indeed, suppose $[a]=1_{[G]}$. Then for an arbitrary $x \in X$ one has $[a x]=[a][x]=[x]$, so

$$
{ }^{x} y={ }^{a x} y={ }^{a}\left({ }^{x} y\right) \quad \forall y \in X .
$$

In particular, (7.16) is true for $y=x$, thus for an arbitrary $x \in X$ one has

$$
x={ }^{x} x={ }^{a x} x={ }^{a}\left({ }^{x} x\right)={ }^{a} x,
$$

where the equality $x={ }^{x} x$ follows from our assumption $(X, r)$ square-free. We have shown ${ }^{a} x=x$, for every $x \in X$, thus $\mathcal{L}_{a}=\mathrm{id} x$. This verifies $N_{0} \subseteq K_{0}$. By Lemma 7.13 the group $K_{0}$ is abelian, so is $N_{0}$.

The equality $H_{0}=K_{1}$ follows from the implications:

$$
\begin{aligned}
u \in H_{0} & \Longleftrightarrow \mathcal{L}_{[u]}=\operatorname{id}_{[X]} \\
& \Longleftrightarrow{ }^{[u]}[x]=\left[{ }^{u} x\right]=[x] \quad \forall x \in X \\
& \Longleftrightarrow{ }^{u} x z=x \quad \forall x, z \in X \\
& \Longleftrightarrow u \in K_{1} .
\end{aligned}
$$

The inclusions (7.13) for the three kernels are clear. This implies the second line in (7.13). The existence of the short exact sequences (7.14) is straightforward from (17.13). One easily sees that the diagram (7.15) is commutative.

We discuss some basic differences between the two kernels $N_{0}$ and $K_{0}$ below.

Remark 7.16. Suppose $(X, r)$ is a nontrivial square-free solution of finite order (so $\mathrm{mpl} X \geq 2$ ). Then $K_{0}$ is a normal subgroup of $G$ of finite index $\left[G: K_{0}\right]$, and in contrast, the index $\left[G: N_{0}\right]$ of $N_{0}$ is not finite. Furthermore, $A \subset K_{0}$, but $A \cap N_{0}=e$. Indeed, by hypothesis $(X, r)$ is a nontrivial solution then, by Lemma 5.2 the set $[X]$ has order $>1$. Furthermore the retract $([X],[r])$ is a braided set. Hence $[X]$ generates the group $G_{1}=G\left([X], r_{[X]}\right),[x] \neq 1_{G_{[X]}}, \forall x \in X$. The group $G_{1}$ is torsion free as a YB group of square-free solution of order $>1$, see Facts 7.11] 
in particular, $\left[x^{p}\right]=[x]^{p} \neq 1_{G_{[X]}}$, so $\forall x \in X, x^{p}$ is not in $N_{0}$. On the other hand we have shown in Lemma 7.13 that $x^{p} \in K_{0}, \forall x \in X$.

The following proposition is an iteration of Lemma 7.15

Proposition 7.17. Let $(X, r)$ be a nontrivial square-free solution. Suppose $\operatorname{mpl}(X, r)=$ $m$. Then the following conditions hold.

(1) For all $j, 0 \leq j \leq m-1$, there are canonical group epimorphisms

$$
\begin{aligned}
\mu_{j}: G_{j} \longrightarrow G_{j+1} & x^{(j)} \mapsto x^{(j+1)} \\
\mathcal{L}_{j}: G_{j} \longrightarrow \mathcal{G}_{j} & x^{(j)} \mapsto \mathcal{L}_{x^{(j)}} \\
f_{j}: G_{j+1} \longrightarrow \mathcal{G}_{j} & x^{(j+1)} \mapsto \mathcal{L}_{x^{(j)}} \\
\varphi_{j}: \mathcal{G}_{j} \longrightarrow \mathcal{G}_{j+1} & \mathcal{L}_{x^{(j)}} \mapsto \mathcal{L}_{x^{(j+1)}}
\end{aligned}
$$

(2) For $0 \leq j \leq m-1$ let $N_{j}$, (respectively, $K_{j}, H_{j}$ ) be the pull-back in $G$ of the kernel $\operatorname{ker} \mu_{j},\left(\right.$ respectively, the pull-back of $\left.\operatorname{ker} \mathcal{L}_{j}, \operatorname{ker} \varphi_{j}\right)$. Then there are inclusions

$$
\begin{aligned}
& N_{0} \subset N_{1} \subset N_{2} \subset \cdots \subset N_{j} \subset N_{j+1} \subset \cdots \\
& \cap \cap \cap \cap \cap \\
& K_{0} \subset K_{1} \subset K_{2} \subset \cdots \subset K_{j} \subset K_{j+1} \subset \cdots \\
& \|\quad\| \quad \| \\
& H_{0} \subset H_{1} \subset \cdots \subset H_{j-1} \subset H_{j} \subset \cdots
\end{aligned}
$$

and

$$
\begin{array}{rlrl}
\operatorname{ker} \mu_{j} & \simeq N_{j} / N_{j-1}, & \operatorname{ker} \mathcal{L}^{j} \simeq K_{j} / N_{j-1} \\
\operatorname{ker} f_{j} \simeq K_{j} / N_{j}, & \operatorname{ker} \varphi_{j} \simeq K_{j+1} / K_{j} .
\end{array}
$$

Remark 7.18. Note that $\operatorname{mpl}(X, r)=m$ if and only if $H_{m-1}=G$.

Remark 7.19. By assumption $(X, r)$ is a square-free solution, thus lri holds and the graph $\Gamma(X, r)$ is well defined. $\mu$ induces a homomorphism of graphs

$$
\mu_{\Gamma}: \Gamma\left(X, r_{X}\right) \longrightarrow \Gamma\left([X], r_{[X]}\right)
$$

The graph $\Gamma\left([X], r_{[X]}\right)$ is a homomorphic image of $\Gamma\left(X, r_{X}\right)$, though not in general a retraction.

Recall that each solvable group $G$ has a canonical solvable series, namely the derived series

$$
G \supset G^{\prime} \supset G^{(2)} \supset \cdots \supset G^{(s)}=1,
$$

where the derived subgroups $G^{(k)}$ are defined recursively. $G^{\prime}$ is the commutator of $G$ (it is generated by the comutators $[x, y]=x y x^{-1} y^{-1}, x, y \in G$ ) and for all $k \geq 1, G^{(k+1)}=\left(G^{(k)}\right)^{\prime}$. Clearly, each $G^{k}$ is a normal subgroup of $G$. The length $s$ of the derived series is called the solvable length of $G$, it is the minimal length of solvable series for $G$. We shall denote the solvable length of $G$ by $\operatorname{sl}(G)$. The following fact is well known, and can be extracted, with a slight modification of the proof, from [MI, Proposition 6.6]. 
TATIANA GATEVA-IVANOVA AND PETER CAMERON

Fact 7.20. Let $N$ be a normal subgroup of $G$, and let $\bar{G}=G / N$. Suppose $N$ and $G / N$ are solvable of solvable lengths $m$ and $s$, respectively. Then the solvable length $\operatorname{sl}(G)$ satisfies $\max (m, s) \leq \operatorname{sl}(G) \leq m+s$.

Remark 7.21. It is known that the $\mathrm{YB}$ group $G(X, r)$ of every finite nondegenerate symmetric set is solvable, see [ESS], Theorem 2.15. In [GI], Theorem 7.10. is given a different proof for the case of finite square-free solutions.

We shall prove that for each multipermutation (square-free) solution $(X, r)$ of arbitrary cardinality the groups $G(X, r)$ and $\mathcal{G}(X, r)$ are solvable, and the solvable length of $G(X, r) \leq \operatorname{mpl}(X, r)$.

Proposition 7.22. Let $(X, r)$ be a square-free solution of arbitrary cardinality, $G=G(X, r), \mathcal{G}=\mathcal{G}(X, r)$. Suppose $\left([X], r_{[X]}\right)=\operatorname{Ret}(X, r), G_{1}=G\left([X], r_{[X]}\right)$. Then the following are equivalent

(1) $G$ is solvable.

(2) $\mathcal{G}$ is solvable.

(3) $G_{1}$ is solvable.

In this case the following inequalities hold:

$$
\operatorname{sl}(\mathcal{G}) \leq \operatorname{sl}\left(G_{1}\right) \leq \operatorname{sl}(G) \leq \operatorname{sl}(\mathcal{G})+1 .
$$

In particular, if some of the retracts $\operatorname{Ret}^{i}(X, r)(i \geq 0$ is a finite set, then $G(X, r)$ is solvable.

Proof. We know that there is a short exact sequence

$$
1 \longrightarrow K_{0} \longrightarrow G \stackrel{\mathcal{L}}{\longrightarrow} \mathcal{G} \longrightarrow 1,
$$

where the kernel $K_{0}=\operatorname{ker} \mathcal{L}$ is an abelian normal subgroup of $G$, see Lemma 7.13. Fact 7.20 implies then that

$$
\operatorname{sl}(G) \leq \operatorname{sl}(\mathcal{G})+1
$$

. By Lemma 7.15 there is a short exact sequence

$$
1 \longrightarrow N_{0} \longrightarrow G \stackrel{\mu_{0}}{\longrightarrow} G_{1} \longrightarrow 1
$$

where the kernel $N_{0}$ of $\mu_{0}$ is an abelian normal subgroup of $G$, so

$$
\operatorname{sl}\left(G_{1}\right) \leq \operatorname{sl}(G) .
$$

By Lemma $7.15 N_{0} \subset K_{0}$ and there is a short exact sequence

$$
1 \longrightarrow K_{0} / N_{0} \longrightarrow G_{1} \longrightarrow \mathcal{G} \longrightarrow 1
$$

thus

$$
\operatorname{sl}(\mathcal{G}) \leq \operatorname{sl}\left(G_{1}\right) .
$$

We have verified the inequalities (7.20). Clearly this implies the equivalence of (1), (2), (3).

Assume now that for some $i$ the retract $\operatorname{Ret}^{i}(X, r)$ is of finite order. Then by Remark 7.21 $G_{i}=G\left(\operatorname{Ret}^{i}(X, r)\right)$ is solvable, and therefore $G_{i-1}=G\left(\operatorname{Ret}^{i-1}(X, r)\right)$ is solvable. By decreasing induction on $i$ we deduce that $G_{0}=G(X, r)$ is solvable. 
Theorem 7.23. Let $(X, r)$ be a square-free solution of arbitrary cardinality, $G=$ $G(X, r), \mathcal{G}=\mathcal{G}(X, r)$. Suppose $(X, r)$ is a multipermutation solution with $\operatorname{mpl}(X, r)=$ $m$. Then $G$ and $\mathcal{G}$ are solvable with

$$
\operatorname{sl}(G) \leq m \quad \text { and } \quad \operatorname{sl}(\mathcal{G}) \leq m-1 .
$$

Furthermore,

$$
\operatorname{mpl}(X, r)=2 \Longrightarrow \operatorname{sl}(G)=2 \quad \text { and } \quad \operatorname{sl}(\mathcal{G})=1
$$

Proof. We shall use induction on $m$ to show that $\operatorname{sl}(G) \leq m$. Note that the retraction $([X],[r])$ is a multipermutation square-free solution of level $\operatorname{mpl}\left([X], r_{[X]}\right)=$ $m-1$. Base for the induction, $m=1$. Then $(X, r)$ is the trivial solution, $\mathcal{G}=e$, and by Lemma 5.2. $G$ is abelian, so $\operatorname{sl} G=1=\operatorname{mpl}(X, r)$.

Suppose the statement is true for $m \leq m_{0}$. Let $\operatorname{mpl}(X, r)=m_{0}+1$. Look at the short exact sequence (7.21). The retraction $\left([X], r_{[X]}\right)$ is a multipermutation square-free solution of level $\operatorname{mpl}\left([X], r_{[X]}\right)=m_{0}$, so by the inductive assumption the solvable length of $G_{1}$ is at most $m_{0}$. Clearly, the solvable length of $N_{0}$ is exactly 1, hence by Fact 7.20 the solvable length of $G$ is at most $m_{0}+1$.

Using analogous argument one shows that $\operatorname{sl} \mathcal{G} \leq m-1$, this time we use the short exact sequence

$$
1 \longrightarrow K_{1} / K_{0} \longrightarrow \mathcal{G} \longrightarrow \mathcal{G}_{1} \longrightarrow 1,
$$

where the kernel $K_{1} / K_{0}$ is an abelian normal subgroup of $\mathcal{G}$, see Lemma 7.15]again. (Here as usual $\mathcal{G}_{1}=\mathcal{G}\left([X], r_{[X])}\right)$. This verifies 7.22

Assume now that $\operatorname{mpl}(X, r)=2$. This implies that $\mathcal{G}(X, r)$ is abelian, or equivalently $\operatorname{sl} \mathcal{G}(X, r)=1$, see Theorem [5.4. We have already shown that $\operatorname{sl} G(X, r) \leq$ $\operatorname{mpl}(X, r)(=2)$. An assumption that there is a strict inequality $\operatorname{sl} G(X, r)<2$ would imply $G(X, r)$ is abelian, and therefore by Lemma $5.2 \operatorname{mpl}(X, r)=1$, a contradiction.

The theorem has been proved.

In the case when $(X, r)$ is of finite order we show that the solvable lengths of $G$ and $\mathcal{G}$ differ exactly with 1 , see Theorem 7.25 .

We need a preliminary lemma.

Lemma 7.24. Let $A$ be a non-zero free abelian group of finite rank, and let $H$ be a non-trivial finite group acting faithfully on $A$. Let

$$
[H, A]=\left\langle{ }^{h} a-a: a \in A, h \in H\right\rangle .
$$

Then $[H, A]$ is non-zero, and $H$ acts faithfully on $[H, A]$.

Proof. We begin by observing that $H$ does indeed act on $[H, A]$. If $k \in H$, then

$$
{ }^{k}\left({ }^{h} a-a\right)={ }^{k h k^{-1}} b-b, \text { where } b={ }^{k} a \in A,
$$

so ${ }^{k}\left({ }^{h} a-a\right) \in[H, A]$.

Let $\hat{A}=A \otimes \mathbb{Q}$. Then $\hat{A}$ is a vector space over $\mathbb{Q}$, with dimension equal to the rank of $A$, and $H$ acts faithfully on $\hat{A}$. It suffices to prove the lemma with $\hat{A}$ in place of $A$, since elements of $[H, A]$ are multiples of elements of $[H, \hat{A}]$. The advantage 
is that Maschke's Theorem holds: if $B$ is an $H$-submodule of $\hat{A}$, then there is a complement $C$, a $H$-submodule such that $\hat{A}=B \oplus C$ (in other words, $\hat{A} / B \cong C)$.

Now $[H, \hat{A}]$ is the smallest $H$-submodule $B$ of $\hat{A}$ such that $H$ acts trivially on $A / B$. So the complement guaranteed by Maschke's Theorem is $C_{H}(\hat{A})=\left\{a \in \hat{A}: a^{h}=\right.$ $a\}$. Since $H \neq\{1\}$ and the action is faithful, $C_{H}(\hat{A}) \neq \hat{A}$, so $[H, \hat{A}] \neq\{0\}$.

Finally, suppose that $h \in H$ acts trivially on $[H, \hat{A}]$. Since also $h$ acts trivially on $C_{H}(\hat{A})$ by definition, it acts trivially on the whole of $\hat{A}$; since we assume that $H$ acts faithfully on $A$, we deduce that $h=1$. The lemma is proved.

Theorem 7.25. Let $(X, r)$ be a square-free solution of finite order. Then

$$
\operatorname{sl}(G)=\operatorname{sl}(\mathcal{G})+1 .
$$

Proof. We know that there is a natural number $p$ such that the subgroup of $G$ generated by the $p$ th powers of the generators is a free abelian group $A$. Clearly $A$ is isomorphic to the integral permutation module for $\mathcal{G}$ (in its action on $X$ ), so the action is faithful. (This uses the equation $b a^{p} b^{-1}=\left({ }^{b} a\right)^{p}$, which follows from $b a^{p}=\left({ }^{b} a\right)^{p} b$, see Facts 7.11 1. i. $)$

Let $A^{(n)}$ be defined inductively by $A^{(0)}=A$ and

$$
A^{(n+1)}=\left[\mathcal{G}^{(n)}, A^{(n)}\right]
$$

for $n \geq 0$. By lemma 7.24 and induction, if $\mathcal{G}^{(n)} \neq\{1\}$, then $A^{(n+1)} \neq\{0\}$ and $\mathcal{G}^{(n)}$ acts faithfully on $A^{(n+1)}$. So, if $l=\operatorname{sl} G$, then $A^{(l)} \neq\{0\}$. But $A^{(l)} \leq G^{(l)}$ (the $l$ th derived group of $G$ ); so $\operatorname{sl} G>l$. By our previous observation, we know that sl $G \leq l+1$; so in fact $\mathrm{sl} G=l+1$ holds, and the theorem is proved.

We know that $\operatorname{mpl}(X, r)=2$ implies $\operatorname{sl}(G(X, r))=2$. Example 5.16 shows that a gap between $\operatorname{mpl}(X)$ and $\operatorname{sl}(G(X, r)$ can occur even for $\operatorname{mpl}(X, r)=3$.

Question 7.26. Suppose $(X, r)$ is a multipermutation square-free solution of finite order $|X|>1$. The following questions are closely related.

(1) Suppose $\operatorname{mpl}(X, r)=m$. Can we express a lower bound for $\operatorname{sl} G(X, r)$ in terms of $m$ ?

(2) When there is an equality?

$$
\operatorname{sl} G(X, r)=\operatorname{mpl}(X, r) \quad \text { or equivalently } \quad \operatorname{sl} \mathcal{G}(X, r)=\operatorname{mpl}(X, r)-1 \text { ? }
$$

In Section 9 we construct an infinite sequence of explicitly defined solutions $\left(X_{m}, r_{m}\right)$, $m=0,1,2 \cdots$, such that $\operatorname{mpl}\left(X_{m}\right)=m$, and $m=\operatorname{sl} G\left(X, r_{m}\right)=\operatorname{sl} \mathcal{G}\left(X, r_{m}\right)+1$, see Definition 9.9 and Theorem 9.11

\section{Wreath PRODUCTS OF SOlUtions}

In this section we define the notion of wreath product of solutions, by analogy with the wreath product of permutation groups.

The following result is true for arbitrary braided sets (without any further restrictions like being symmetric, finite, or square-free). 
Theorem 8.1. GIM08 Let $\left(X, r_{X}\right),\left(Y, r_{Y}\right)$ be disjoint solutions of the $Y B E$, with $Y B$ - groups $G_{X}=G\left(X, r_{X}\right)$, and $G_{Y}=G\left(Y, r_{Y}\right)$. Let $(Z, r)$ be a regular $Y B$ extension of $\left(X, r_{X}\right),\left(Y, r_{Y}\right)$, with a $Y B$-group $G_{Z}=G(Z, r)$. Then

- $G_{X}, G_{Y}$ is a matched pair of groups with actions induced from the braided group $\left(G_{Z}, r\right)$.

- $G_{Z}$ is isomorphic to the double crossed product $G_{X} \bowtie G_{Y}$.

Fact 8.2. [ESS] Let $\left(X, r_{X}\right)$ and $\left(Y, r_{Y}\right)$ be symmetric sets. If $Z \in \operatorname{Ext}^{+}(X, Y)$, then $G_{Z} \simeq G_{Y} \rtimes G_{X}$, where the semidirect product is formed using the action of $G_{Y}$ on $X$ via $\alpha \rightarrow \mathcal{L}_{\alpha}$.

$\operatorname{Ext}^{+}(X, Y)$ is the set of all symmetric sets $(Z, r)$ which are extensions of $\left(X, r_{1}\right),\left(Y, r_{2}\right)$ with $r(x, \alpha)=\left(\alpha, x^{\alpha}\right)$.

Lemma 8.3. Let $(X, r)$ be square-free multipermutation solution, let $\tau \in \operatorname{Aut}(X, r)$ be an automorphism of $(X, r)$. Let $\left(Y, r_{0}\right)$ be the trivial solution on the one element set $Y=\{\alpha\}$, where $\alpha$ is not in $X$. Let $\left(Z, r_{Z}\right)=X \boxminus Y$ be the strong twisted union defined via $\mathcal{L}_{\alpha}=\tau, \mathcal{L}_{x \mid Y}=\operatorname{id}_{Y}$, for all $x \in X$. (i.e. ${ }^{\alpha} x=\tau(x),{ }^{x} \alpha=\alpha$, for all $x \in X$.) Then

(1) $\left(Z, r_{Z}\right)$ is a square-free solution, so $Z=X \downarrow Y$.

(2) $G\left(Z, r_{Z}\right)$ is the semidirect product $G\left(Z, r_{Z}\right) \simeq G(X, r) \rtimes C_{\infty}$

(3) Furthermore, if $(X, r)$ is a multipermutation solution of finite multipermutation level, and $\tau$ does not belong to $\mathcal{G}(X, r)$ then

$$
\operatorname{mpl}\left(Z, r_{Z}\right)=\operatorname{mpl}(X, r)+1 \text {. }
$$

The following proposition can be deduced from GIM08.

Proposition 8.4. Let $\left(X, r_{x}\right),\left(Y, r_{Y}\right)$ be disjoint nondegenerate symmetric sets (not necessarily finite or square-free). Assume there is an injective map $Y \longrightarrow$ $\operatorname{Sym}(X), \alpha \mapsto \sigma_{\alpha} \in \operatorname{Sym}(X)$. Let $(Z, r)$ be the extension of $\left(X, r_{X}\right)$, and $\left(Y, r_{Y}\right)$ where $Z=X \bigcup Y, r$ extends $r_{X}$ and $r_{Y}$, and

$$
r(\alpha, x)=\left(\sigma_{\alpha}(x), \alpha\right) \quad r(x, \alpha)=\left(\alpha, \sigma_{\alpha}^{-1}(x)\right) \quad \forall x \in X, \alpha \in Y .
$$

Then $(Z, r)$ is a symmetric set if and only if the assignment $\alpha \mapsto \sigma_{\alpha}$ extends to a homomorphism $L_{Y}: G\left(Y, r_{Y}\right) \longrightarrow \operatorname{Aut}\left(X, r_{X}\right)$.

Lemma 8.5. Suppose $\left(X, r_{y}\right),\left(Y, r_{Y}\right)$ are disjoint symmetric sets (most general setting). Let $\sigma \in \operatorname{Sym} X, \rho \in \operatorname{Sym} Y$. Let $(Z, r)$, be an extension of $\left(X, r_{X}\right),\left(Y, r_{Y}\right)$, such that

$$
r(x, y)=\left(\rho(y), \sigma^{-1}(x)\right) \quad r(y, x)=\left(\sigma(x), \rho^{-1}(y)\right)
$$

Then $(Z, r)$ is involutive, nondegenerate quadratic set. Furthermore, $(Z, r)$ is a solution if and only if $\sigma \in \operatorname{Aut} X$ and $\rho \in \operatorname{Aut} Y$.

We now define the wreath product of solutions:

Definition 8.6. Suppose $\left(X_{0}, r_{0}\right)$, and $\left(Y, r_{Y}\right)$ are disjoint square-free solutions. Let $\left\{\left(X_{\alpha}, r_{\alpha}\right) \mid \alpha \in Y\right\}$ be the set of $|Y|$ disjoint solutions $\left(X_{\alpha}, r_{\alpha}\right)$ indexed by $Y$, where each $\left(X_{\alpha}, r_{\alpha}\right)$ is an isomorphic copy of $\left(X_{0}, r_{0}\right)$ defined on a set $X_{\alpha}=\left\{t_{\alpha, x} \mid\right.$ $\left.x \in X_{0}\right\}$, and $t_{\alpha, x}$ denotes the copy of $x$ in $X_{\alpha}\left(t_{\alpha, x} \neq t_{\alpha, z}\right.$ if and only if $\left.x \neq z\right)$, 
and the map $r_{\alpha}$ and the associated actions are translations of $r_{0}$ and its associated actions to $X_{\alpha}$. Thus

$$
r_{\alpha}\left(t_{\alpha, x}, t_{\alpha, z}\right)=\left(t_{(\alpha, x z)}, t_{\left(\alpha, x^{z}\right)}\right) \quad \forall x, z \in X .
$$

Let $\left(X, r_{X}\right)=\natural_{\alpha \in Y} X_{\alpha}$, be the trivial extension of all $X_{\alpha}$, for $\alpha \in Y$; that is, for all $\alpha, \beta \in Y$, we have

$$
\begin{gathered}
r_{\mid X_{\alpha} \times X_{\alpha}}=r_{\alpha} \\
r(x, z)=(z, x), \forall x \in X_{\alpha}, z \in X_{\beta}, \text { with } \alpha \neq \beta
\end{gathered}
$$

Define the map $Y \longrightarrow \operatorname{Sym} X, \quad \beta \mapsto \sigma_{\beta}$, where the permutations $\sigma_{\beta} \in \operatorname{Sym} X$ are defined as follows

$$
\begin{gathered}
\sigma_{\beta}: X_{\alpha} \longrightarrow X_{\beta}, \quad \alpha \in Y \\
\sigma_{\beta}\left(t_{\alpha, x}\right)=t_{(\beta \alpha), x}
\end{gathered}
$$

Let $(Z, r)$ be the extension of $\left(X, r_{X}\right)$ and $\left(Y, r_{Y}\right)$ defined as follows

$$
\begin{aligned}
Z= & X \bigcup Y, \\
r\left(\beta,\left(t_{\alpha, x}\right)\right)= & \left(\sigma_{\beta}\left(t_{\alpha, x}\right), \beta\right), \\
r\left(\left(t_{\alpha, x}\right), \beta\right)= & \left(\beta,\left(\sigma_{\beta}\right)^{-1}\left(t_{\alpha, x}\right)\right), \\
& \forall \alpha, \beta \in Y, \quad t_{\alpha, x} \in X_{\alpha} .
\end{aligned}
$$

We call $(Z, r)$ a wreath product of $\left(X_{0}, r_{X_{0}}\right)$ and $\left(Y, r_{Y}\right)$, and denote it by $(Z, r)=$ $\left(X_{0}, r_{X_{0}}\right) \operatorname{wr}\left(Y, r_{Y}\right)$.

Theorem 8.7. (1) The wreath product $(Z, r)=\left(X_{0}, r_{X_{0}}\right) \mathrm{wr}\left(Y, r_{Y}\right)$ is a squarefree solution.

$$
\mathcal{G}(Z, r)=\mathcal{G}\left(X_{0}, r_{X_{0}}\right) \text { wr } \mathcal{G}\left(Y, r_{Y}\right) .
$$

(3) Suppose $\left(X_{0}, r_{X_{0}}\right)$ and $\left(Y, r_{Y}\right)=$ are multipermutation solutions of finite multipermutation level. Then

$$
\operatorname{mpl}(Z, r)=\operatorname{mpl}\left(X_{0}, r_{X_{0}}\right)+\operatorname{mpl}\left(Y, r_{Y}\right)-1 .
$$

Proof. Note that $(Z, r)$ satisfies the hypothesis of Proposition 8.4 , hence $(Z, r)$ is a solution if and only if the map $Y \longrightarrow \operatorname{Sym} X, \quad \beta \mapsto \sigma_{\beta}$, extends to a homomorphism $L_{Y}: G\left(Y, r_{Y}\right) \longrightarrow \operatorname{Aut}\left(X, r_{X}\right)$.

We show first that

$$
\sigma_{\beta} \in \operatorname{Aut}\left(X, r_{X}\right) \quad \forall \beta \in Y .
$$

By Lemma 3.3 this is equivalent to

$$
\sigma_{\beta} \circ \mathcal{L}_{t_{\alpha, x}}=\left(\mathcal{L}_{\sigma_{\beta}\left(t_{\alpha, x}\right)}\right) \circ \sigma_{\beta} \quad \forall \alpha .
$$

Note that by the definition of $r$ the associated left action on $Z$ satisfies:

$$
\mathcal{L}_{t_{\alpha, x}}\left(t_{\gamma, y}\right)= \begin{cases}\left.t_{(\alpha, x} y\right) & \text { if } \gamma=\alpha \\ t_{\gamma, y} & \text { else }\end{cases}
$$


Let $t_{\gamma, y} \in X$. We apply both sides of (8.3) on $t_{\gamma, y}$, and compute using (8.4) and (8.11). Case 1. $\gamma=\alpha$.

$$
\begin{aligned}
\sigma_{\beta} \circ \mathcal{L}_{t_{\alpha, x}}\left(t_{\alpha, y}\right) & =\sigma_{\beta}\left(t_{\alpha, x} y\right) \\
& =t_{\beta}{ }^{x,{ }^{x} y} \\
\mathcal{L}_{\sigma_{\beta}}\left(t_{\alpha, x}\right) \circ \sigma_{\beta}\left(t_{\alpha, y}\right) & =\mathcal{L}_{t_{(\beta \alpha, x)}}\left(t_{\beta} \alpha, y\right) \\
& =t_{\left({ }^{\beta} \alpha,{ }^{x} y\right)},
\end{aligned}
$$

as desired. Case 2. $\gamma \neq \alpha$. In this case, due to the nondegeneracy of $r_{Y}$ one has

$$
{ }^{\beta} \alpha \neq{ }^{\beta} \gamma, \quad \forall \beta \in Y \text {. }
$$

Now our computation (applying (8.4), (8.1), and (8.6)) give:

$$
\begin{aligned}
\sigma_{\beta} \circ \mathcal{L}_{t_{\alpha, x}}\left(t_{\gamma, y}\right) & =\sigma_{\beta}\left(t_{\gamma, y}\right) \\
& =t_{\beta} \gamma, y \\
\mathcal{L}_{\sigma_{\beta}\left(t_{\alpha, x}\right)} \circ \sigma_{\beta}\left(t_{\gamma, y}\right) & =\mathcal{L}_{\left(t_{\beta, x}\right)}\left(t_{\beta} \gamma, y\right) \\
& =t_{\left({ }^{\beta} \gamma, y\right)} .
\end{aligned}
$$

We have verified (8.3), therefore (8.2) holds.

Next we show that the map $L_{0}: Y \longrightarrow \operatorname{Aut}(X, r) \quad \beta \mapsto \sigma_{\beta}$ extends to a homomporphism

$$
G\left(Y, r_{Y}\right) \longrightarrow \operatorname{Aut}(X, r) .
$$

$Y$ generates $G\left(Y, r_{Y}\right)$, so it will be enough to show that $L_{0}$ respects the relations of $G\left(Y, r_{Y}\right)$, or equivalently

$$
\sigma_{\beta} \circ \sigma_{\gamma}=\sigma_{\beta} \circ \sigma_{\beta^{\gamma}}, \quad \forall \beta, \gamma \in Y .
$$

Recall that since $\left(Y, r_{Y}\right)$ is a solution, condition $\mathbf{1 1}$ holds, that is

$$
{ }^{\beta}\left({ }^{\gamma} \alpha\right)={ }^{\beta} \gamma\left(\beta^{\gamma} \alpha\right) \quad \forall \alpha, \beta, \gamma \in Y .
$$

Let $t \in X$. Then $t=t_{\alpha, x}$ for some $\alpha \in Y, x \in X_{0}$. We apply both sides of (8.8) on $t$ and obtain:

$$
\begin{aligned}
\sigma_{\beta} \circ \sigma_{\gamma}\left(t_{\alpha, x}\right) & =\sigma_{\beta}\left(t_{\gamma} \alpha, x\right) \\
& =t_{(\beta(\gamma \alpha), x)} \\
& =t_{\left({ }^{\beta} \gamma\left({ }^{\beta \gamma} \alpha\right), x\right)} \\
\sigma_{\beta} \circ \sigma_{\beta \gamma}\left(t_{\alpha, x}\right) & =t_{\left({ }^{\beta} \gamma\left(\beta^{\beta} \alpha\right), x\right)}
\end{aligned}
$$

which verifies (8.7). We have shown that the sufficient conditions for $(Z, r)$ being a solution are satisfied which proves part (1) of the theorem.

(2) The wreath product of a group $G$ by a permutation group $H$ (acting on the set $Y$ ) is defined to be the semidirect product of $N$ by $H$, where $N$ (the base group) is the direct product of $|Y|$ copies of $G$, and $H$ acts on $N$ by permuting the direct factors in the same way as it permutes the indexing elements of $Y$. If $G$ is itself a permutation group on a set $X$, then $G$ wr $H$ is in a natural way a permutation group on $X \times Y$ (regarded as the disjoint union of $|Y|$ copies of $X$ ).

It is clear from our construction that $\mathcal{G}(Z, r)$ is isomorphic as abstract group to $\mathcal{G}\left(X_{0}, r_{X_{0}}\right)$ wr $\mathcal{G}\left(Y, r_{Y}\right)$, and in fact acts on the union of copies of $X_{0}$ by the natural permutation action of the wreath product; it acts on $Y$ according to the action of $\mathcal{G}\left(Y, r_{Y}\right)$ (that is, the base group is in the kernel of this action). 
(3) Suppose now that $\left(X_{0}, r_{0}\right)$, and $\left(Y, r_{Y}\right)$ are multipermutation solutions with $\operatorname{mpl}\left(X_{0}, r_{0}\right)=m$ and $\operatorname{mpl}\left(Y, r_{Y}\right)=n$. Every retract $\operatorname{Ret}^{k}(X, r)$ is a trivial extension of the retracts $\operatorname{Ret}^{k}\left(X_{\alpha}, r_{\alpha}\right), \alpha \in Y$. It follows straightforwardly that $\operatorname{mpl}\left(X, r_{X}\right)=$ $\operatorname{mpl}\left(X_{0}, r_{0}\right)=m$

We study the retracts $\operatorname{Ret}^{N}(Z, r), N=1,2, \cdots$. Note first that by the definition of the map $r$ on $Z \times Z$ it follows that

$$
\begin{gathered}
\mathcal{L}_{x \mid X}=\mathcal{L}_{y \mid X} \Longleftrightarrow \mathcal{L}_{x \mid Z}=\mathcal{L}_{y \mid Z} \quad x, y \in X \\
\mathcal{L}_{\alpha \mid Y}=\mathcal{L}_{\beta \mid Y} \Longleftrightarrow \mathcal{L}_{\alpha \mid Z}=\mathcal{L}_{\beta \mid Z} \quad \alpha, \beta \in Y .
\end{gathered}
$$

Hence the retract $\operatorname{Ret}(Z, r)$ can be viewed as the extension of $\operatorname{Ret}\left(X, r_{X}\right)$ and $\operatorname{Ret}\left(Y, r_{Y}\right)$, induced from the original actions of $Y$ onto $X$, but reduced after collapsing all elements $x \in X$, with $\mathcal{L}_{x}=\mathrm{id}_{X}$ and $\alpha \in Y$, with $\mathcal{L}_{\alpha}=\mathrm{id}_{Y}$ into a single point, say $\left[z_{0}\right]$. This does not have effect on $\operatorname{Ret}\left(X, r_{X}\right), \operatorname{Ret}\left(Y, r_{Y}\right)$, but now these solutions might intersect in a single joint point (with trivial action). For each $k, 1 \leq k \leq m, \alpha \in Y$, as usual, (see Notation 7.1) $\alpha^{(k)}$ denotes the equivalence class of $\alpha$ in $\operatorname{Ret}^{k}(Z, r)$. Denote $Y_{k}=\left\{\alpha^{(k)} \mid \alpha \in Y\right\}$. Note that $\left(Y_{k}, r_{k}\right)$ (with $r_{k}$ induced from $\left.r_{Y}\right)$ is isomorphic to $\operatorname{Ret}\left(Y, r_{Y}\right)=\left([Y], r_{[Y]}\right.$ for all $k, 1 \leq k \leq m$. Note also that each retract $\operatorname{Ret}^{k}(Z, r)$, with $1 \leq k \leq m-1$ is an union of $\operatorname{Ret}^{k}\left(X, r_{X}\right)$ and $\left(Y_{k}, r_{Y_{k}}\right)$ with possibly one joint point $z_{0}^{(k)}$, the class of all $\xi \in Z$ which act trivially on $\operatorname{Ret}^{k-1}(Z, r)$.

Indeed every such a retraction has the effect of obtaining the retractions $\operatorname{Ret}^{k}\left(X_{\alpha}, r_{\alpha}\right)$, where $\alpha \in Y$, but these are disjoint sets and therefore for each pair $\alpha, \beta \in Y$ the inequality of equivalence classes in $Y,[\alpha] \neq[\beta]$ implies $\left(\mathcal{L}_{[\alpha]}\right)_{\mid[X]} \neq\left(\mathcal{L}_{[\beta]}\right)_{\mid[X]}$, and therefore $\left(\mathcal{L}_{[\alpha]}\right)_{\mid[Z]} \neq\left(\mathcal{L}_{[\beta]}\right)_{\mid[Z]}$.

This way exactly on the $m$-th retraction $\operatorname{Ret}^{m}(Z, r)$ all the elements of $X$ collapse in a single element $z_{0}^{(m)}$ (which could be also the unique joint element with $Y_{m}$ ). From now on the $m+j$-th retraction $\operatorname{Ret}^{m+j}(Z, r)$ has effect of $j+1$-th retraction on $\left(Y, r_{Y}\right)$ in the usual way, since as we know $\left(Y_{m}, r_{m}\right)$ is isomorphic to $\operatorname{Ret}\left(Y, r_{Y}\right)$, all element of $Y_{m}$ have trivial action on $z_{0}^{(m)}$. It follows than that we need exactly $n-2$ more retractions to obtain that $\operatorname{Ret}^{m+n-2}(Z, r)$ is a trivial solution of order $\geq 2$, hence $\operatorname{Ret}^{m+n-1}(Z, r)=\left\{z_{0}^{(m+n-1)}\right\}$, is a one element solution. This verifies (3).

Open questions 8.8. Suppose the square-free solution $(Z, r)=X \boxminus Y$ is a strong twisted union of $\left(X, r_{X}\right)$ and $\left(Y, r_{Y}\right)$. . Denote $G_{Z}=G(Z, r), G_{X}=G\left(X, r_{X}\right), G_{Y}=$ $G\left(Y, r_{Y}\right)$

1) How are the groups $G_{Z}, G_{X}, G_{Y}$ related?

Proposition 4.6. GIM08, shows that for arbitrary braided set $(Z, r)$ which is an extension of two disjoint sets $\left(X, r_{X}\right)$ and $\left(Y, r_{Y}\right), G_{X}, G_{Y}$ is a matched pair of groups and $G_{Z}$ is isomorphic to the double crossed product $G_{X} \bowtie G_{Y}$.

Note that in the case when $Z$ is a strong twisted union of $X Y$, the group $G_{X}$ acts on $G_{Y}$ via automorphisms and $G_{Y}$, acts on $G_{X}$ vis automorphisms, so we expect the structure of $G_{Z} \simeq G_{X} \bowtie G_{Y}$ to be more special.

2) How are the groups $\mathcal{G}(Z, r), \mathcal{G}\left(X, r_{X}\right), \mathcal{G}\left(Y, r_{Y}\right)$ related? 
3) Can we determine a upper bound for the solvable length of $G(Z, r)$ in terms of the solvable lengths of $G_{X}, G_{Y}$. Analogous question for the solvable lengths of $\mathcal{G}(Z, r), \mathcal{G}_{X}, \mathcal{G}_{Y}$

Moreover, suppose both $\left(X, r_{X}\right)$ and $\left(Y, r_{Y}\right)$, are multipermutation solutions.

4) Is it true that $(Z, r)$ is always a multipermutation solution?

5) How is $\operatorname{mpl}(Z)$ related to $\operatorname{mpl}(X), \operatorname{mpl}(Y)$ ?

Clearly $\max (\operatorname{mpl} X, \operatorname{mpl} Y) \leq \operatorname{mpl}(Z)$. Can we express an upper bound for $\operatorname{mpl}(Z)$ in terms of $\operatorname{mpl}(X), \operatorname{mpl}(Y)$ ?

6) Suppose $(Z, r)$ is a finite multipermutation square-free solution, with $\operatorname{mpl} Z=m$. Is it true that $(Z, r)$ can be always presented as a strong twisted union of $r$-invariant subsets of multipermutation level $<m$ ?

Lemma 8.9. There exist two disjoint square-free symmetric sets $\left(X, r_{X}\right),\left(Y, r_{Y}\right)$, with $\operatorname{mpl}(X)=\operatorname{mpl}(Y)=2$, and a strong twisted union $(Z, r)=X \boxminus Y$, which is a multipermutation solution with $\operatorname{mpl}(Z, r)=\operatorname{mpl}(X)+\operatorname{mpl}(Y)=4$. Moreover, $\operatorname{sl}(G(Z, r))=4=\operatorname{sl}\left(G_{X}\right)+\operatorname{sl}\left(G_{Y}\right)$, and $\operatorname{sl}(\mathcal{G}(Z, r))=3$.

Proof. We shall define $\left(X, r_{X}\right),\left(Y, r_{Y}\right)$ and $(Z, r)$ explicitly, via the left actions. Let $\left(X, r_{X}\right),\left(Y, r_{Y}\right)$ be the solutions defined as

$$
\begin{array}{cl}
X=\bigcup_{1 \leq i \leq 4} X^{i} & X^{i}=\left\{x_{1}^{i}, x_{2}^{i}, x_{3}^{i}, x_{4}^{i}\right\}, 1 \leq i \leq 4 \\
\mathcal{L}_{x_{1}^{i}}=\mathcal{L}_{x_{3}^{i}}=\left(x_{2}^{i} x_{4}^{i}\right) & \mathcal{L}_{x_{2}^{i}}=\mathcal{L}_{x_{4}^{i}}=\left(x_{1}^{i} x_{3}^{i}\right), 1 \leq i \leq 4 . \\
Y=\left\{a_{1}, a_{2}, a_{3}, a_{4}, a_{1}^{\prime}, a_{2}^{\prime}, a_{3}^{\prime}, a_{4}^{\prime}, b, c\right\}, & \mathcal{L}_{a_{i}}=\mathcal{L}_{a_{i}^{\prime}}=\mathrm{id}_{Y}, \quad 1 \leq i \leq 4, \\
\mathcal{L}_{b}=\left(a_{1} a_{2}\right)\left(a_{3} a_{4}\right)\left(a_{1}^{\prime} a_{2}^{\prime}\right)\left(a_{3}^{\prime}, a_{4}^{\prime}\right), & \mathcal{L}_{c}=\left(a_{1} a_{3}\right)\left(a_{2} a_{4}\right)\left(a_{1}^{\prime} a_{3}^{\prime}\right)\left(a_{2}^{\prime} a_{4}^{\prime}\right) .
\end{array}
$$

Note that $X$ is a trivial extension of the four isomorphic solutions $\left(X^{i}, r_{i}\right), 1 \leq i \leq 4$, with $r_{i}$ defined, as usual, via the left action. It is easy to see that $\operatorname{mpl} X^{i}=2$ Clearly, one has mpl $X=2$. Direct computation shows that $\operatorname{mpl} Y=2$. We set $Z=X \bigcup Y$. In (8.11) we define a left action $Z \times Z \longrightarrow Z$ extending the given actions $X \times X \longrightarrow X$ and $Y \times Y \longrightarrow Y$. (All permutations bellow are considered as elements of $\operatorname{Sym} Z$ ).

$$
\begin{aligned}
& \mathcal{L}_{x_{1}^{i}}=\mathcal{L}_{x_{3}^{i}}=\left(x_{2}^{i} x_{4}^{i}\right)\left(a_{i} a_{i}^{\prime}\right) \quad \mathcal{L}_{x_{2}^{i}}=\mathcal{L}_{x_{4}^{i}}=\left(x_{1}^{i} x_{3}^{i}\right)\left(a_{i} a_{i}^{\prime}\right), \\
& \mathcal{L}_{a_{i}}=\mathcal{L}_{a_{i}^{\prime}}=\left(x_{1}^{i} x_{2}^{i} x_{3}^{i} x_{4}^{i}\right), \quad 1 \leq i \leq 4 ; \\
& \mathcal{L}_{b}=\left(a_{1} a_{2}\right)\left(a_{3} a_{4}\right)\left(a_{1}^{\prime} a_{2}^{\prime}\right)\left(a_{3}^{\prime} a_{4}^{\prime}\right) \prod_{1 \leq i \leq 4}\left[\left(x_{i}^{1} x_{i}^{2}\right)\left(x_{i}^{3} x_{i}^{4}\right)\right], \\
& \mathcal{L}_{c}=\left(a_{1} a_{3}\right)\left(a_{2} a_{4}\right)\left(a_{1}^{\prime} a_{3}^{\prime}\right)\left(a_{2}^{\prime} a_{4}^{\prime}\right) \prod_{1 \leq i \leq 4}\left[\left(x_{i}^{1} x_{i}^{3}\right)\left(x_{i}^{2} x_{i}^{4}\right)\right] .
\end{aligned}
$$

Consider the map $r: Z \times Z \longrightarrow Z \times Z$, defined as $r(z, t)=\left(\mathcal{L}_{z}(t), \mathcal{L}_{t}^{-1}(z)\right)$ where $t, z \in Z, \mathcal{L}_{z}, \mathcal{L}_{t}$ as in (8.11). One verifies straightforwardly condition 11, hence $(Z, r)$ is a symmetric set. Furthermore, condition stu holds, so $(Z, r)=\left(X, r_{X}\right) \natural\left(Y, r_{Y}\right)$. Direct computation shows that $\operatorname{mpl} Z=4$. Moreover, a short calculation with GAP GAP shows that the group $\mathcal{G}(Z, r)$ has order $2^{14}$ and solvable length 3 . (This can easily be seen directly, since $\mathcal{G}$ is the wreath product of the dihedral group of order 8 with the Klein group of order 4.) By Theorem 7.25, $\mathrm{sl} G=4$. 


\section{INFINITE SOLUTIONS}

We consider solutions $(X, r)$ with $X$ infinite but having finite multipermutation level.

Question 9.1. In the hypothesis and notation of Lemma 8.3, when can we express $\mathcal{G}(Z, r)$ ) as a wreath product $\mathcal{G}(X, r) \mathrm{wr}\left\langle\tau^{\prime}\right\rangle$ (where $\tau^{\prime}$ is some appropriate permutation deduced from $\tau)$ ? (This is not true in general.)

Note that, in general, $\operatorname{Aut}(X, r) \subseteq \mathcal{G}(X, r)$, is possible (so the existence of a $\tau \notin$ $\mathcal{G}(X, r)$ is not automatic), as show the following example.

Example 9.2. Let $(X, r)$ be the three element nontrivial solution, with $X=$ $\left\{x_{1}, x_{2}, x_{3}\right\}$ and $r$ defined via the left actions $\mathcal{L}_{x_{3}}=\left(x_{1} x_{2}\right), \mathcal{L}_{x_{1}}=\mathcal{L} x_{2}=\operatorname{id}_{X} x$. Then $\operatorname{mpl}(X, r)=2$ and $\operatorname{Aut}(X, r)=\mathcal{G}(X, r)=\left\{i d_{X},\left(x_{1} x_{2}\right)\right\}$,

Example 9.3. We shall construct an infinite sequence of explicitly defined squarefree symmetric sets

$$
\left(X_{0}, r_{0}\right),\left(X_{1}, r_{1}\right), \cdots,\left(X_{m}, r_{m}\right), \cdots
$$

such that

(i) for each $m, m=0,1,2, \cdots, X_{m} \subset X_{m+1}$ is an $r_{m+1}$-invariant subset of $X_{m+1}$. Furthermore,

(ii) $\left(X_{m}, r_{m}\right)$ is a finite multipermutation solution of order $|X|=2^{m+1}-1$, and

(iii) $\operatorname{mpl}\left(X_{m}, r_{m}\right)=m$, the solvable length of each $\mathcal{G}\left(X_{m}, r_{m}\right)$ is exactly $m$.

(iv) $\left(X_{m+1}, r_{m+1}\right.$ is a strong twisted union of a solution $\left(Y_{m}, r_{m}\right)$, with $\operatorname{mpl}\left(Y_{m}\right)=$ $m,\left|Y_{m}\right|=2\left(2^{m}-1\right)$, and a trivial one-element solution.

As a starting point we chose an infinite countable set $X=\left\{x_{n} \mid 1 \leq n\right\}$. We define the solutions $\left(X_{m}, r_{m}\right), m=0,1,2, \cdots$ recursively.

- $\left(X_{0}, r_{0}\right)$ is the one element trivial solution with $X_{0}=\left\{x_{1}\right\}$.

- $\left(X_{1}, r_{1}\right)$ is the trivial solution on the set $X_{1}=\left\{x_{1}, x_{2}\right\}$.

- We set $\left(X_{2}, r_{2}\right)=X_{1} \natural\left\{x_{3}\right\}$, where $\mathcal{L}_{x_{3}}=\left(x_{1} x_{2}\right)$. Clearly, mpl $X_{2}=2$.

- Construction of $\left(X_{3}, r_{3}\right)$. Let $\left(X_{2}^{\prime}, r_{2}^{\prime}\right)$ be an isomorphic copy of $\left(X_{2}, r_{2}\right)$, where $X_{2}^{\prime}=\left\{x_{4}, x_{5}, x_{6}\right\}$, and the map $\tau:\left(X_{2}, r_{2}\right) \longrightarrow\left(X_{2}^{\prime}, r_{2}^{\prime}\right)$ with $\tau\left(x_{i}\right)=$ $x_{i+3}, 1 \leq i \leq 3$, is an isomorphism of solutions. Let $\left(Y_{2}, r_{Y_{2}}\right)=X_{2} \natural_{0} X_{2}^{\prime}$ be the trivial extension. We set $\left(X_{3}, r_{3}\right)=Y_{2} \curvearrowleft\left\{x_{7}\right\}$, where the map $r_{3}$ is defined via the left action $\mathcal{L}_{x_{7}}=\left(x_{1} x_{4}\right)\left(x_{2} x_{5}\right)\left(x_{3} x_{6}\right)$. One has $\mathcal{L}_{x_{7}} \in$ $\operatorname{Aut}\left(Y_{2}, r_{Y_{2}}\right) \backslash \mathcal{G}\left(Y_{2}, r_{Y_{2}}\right)$, so $\mathrm{mpl} X_{3}=3$.

Assume we have constructed the sequence $\left(X_{0}, r_{0}\right),\left(X_{1}, r_{1}\right), \cdots,\left(X_{m}, r_{m}\right)$, satisfying conditions (i) and (ii). We shall construct effectively $\left(X_{m+1}, r_{m+1}\right)$ so that (i), (ii). and (iii) are satisfied. For $N=2^{m}-1=\left|X_{m}\right|$, let $X_{m}^{\prime}=\left\{x_{N+1}, \cdots x_{2 N},\right\}$ and let $\left(X_{m}^{\prime}, r_{X_{m}^{\prime}}\right)$ be the solution isomorphic to $\left(X_{m}, r_{m}\right)$ via the isomorphism $\tau: X_{m} \longrightarrow X_{m}^{\prime}$ with $\tau\left(x_{i}\right)=x_{i+N}, 1 \leq i \leq N$. We denote by $\left(Y_{m}, r_{Y_{m}}\right)$ the trivial extension $X_{m} \natural_{0} X_{m}^{\prime}$, and set $X_{m+1}=\left(Y_{m}, r_{Y_{m}}\right) \natural\left\{x_{2 N+1}\right\}$, where $r_{m+1}$ is defined via the action $\mathcal{L}_{x_{2 N+1}}=\left(x_{1} x_{N+1}\right)\left(x_{2} x_{N+2}\right) \cdots\left(x_{N} x_{2 N}\right)$. One can show that $\operatorname{mpl}\left(X_{m+1}, r_{m+1}\right)=m+1$, and $\mathcal{G}\left(X_{m+1}, r_{m+1}\right)=\mathcal{G}\left(X_{m}, r_{m}\right)$ wr $C_{2}$. 
The following question was posed by Paul Martin.

Question 9.4. For each positive integer $m$ denote by $\mathbf{n}_{m}$, the minimal integer so that there exists a square-free multipermutation solution $\left(X_{m}, r_{m}\right)$ of order $\left|X_{m}\right|=$ $\mathbf{n}_{m}$, and with $\operatorname{mpl}\left(X_{m}, r_{m}\right)=m$. How does $\mathbf{n}_{m}$ depend on $m$ ?

In the proof of Theorem 9.11 we construct an infinite sequence of recursively defined explicit solutions $\left(X_{m}, r_{m}\right), m=0,1,2 \cdots$, s.t. $\operatorname{mpl}\left(X_{m}\right)=m$, and $\left|X_{m}\right|=$ $2^{m-1}+1$. Therefore $\mathbf{n}_{m} \leq 2^{m-1}+1$. By definition, the unique symmetric set with multipermutation level 0 is the one element solution, so $\mathbf{n}_{0}=1$. Direct computation show that for $1 \leq m \leq 3$ the square-free solutions $\left(X_{m}, r_{m}\right)$ in the Construction 9.11, are of minimal possible order, so $\mathbf{n}_{m}=2^{m-1}+1, m=1,2,3$.

Question 9.5. Is it true that $\mathbf{n}_{m}=2^{m-1}+1$, for all integers $m \geq 1$ ?

Let $\mathbb{X}=\left\{x_{i} \mid i=1,2,3, \cdots\right\}$ be an infinite countable set.

Definition 9.6. Let $\rho=\left(x_{i_{1}} x_{i_{2}} \cdots x_{i_{k}}\right)$ and $\sigma=\left(y_{i_{1}} y_{i_{2}} \cdots y_{i_{k}}\right)$ be disjoint cycles of length $k$ in $\operatorname{Sym}(X)$, and let $N$ be a natural number. Define

$$
\begin{aligned}
& \rho[N]=\left(\begin{array}{llll}
x_{i_{1}+N} & x_{i_{2}+N} & \cdots & x_{i_{k}+N}
\end{array}\right)
\end{aligned}
$$

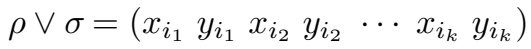

So $\rho[N]$ is a cycle of the same length $k$ as $\rho$ and is obtained by shifting the indices $N$-steps to the right. $\rho \vee \sigma$ is a cycle of length $2 k$ and

$$
(\rho \vee \sigma)^{2}=\rho \circ \sigma \text {. }
$$

Definition 9.7. For a pair $\left(x_{i}, x_{j}\right) \in \mathbb{X} \times \mathbb{X}$ we define $\left(x_{i}, x_{j}\right)[N]=\left(x_{i+N}, x_{j+N}\right)$. Let $(Y, r)$ be a symmetric set, where $Y=\left\{x_{1}, x_{2} \cdots x_{k}\right\} \subset \mathbb{X}$. For each integer $N, N>k$ we define the quadratic set $(Y, r)[N])=(Y[N], r[N])$, where $Y[N]=$ $\left\{x_{1+N}, x_{2+N} \cdots x_{k+N}\right\}$, and

$$
r[N]\left(x_{i+N}, x_{j+N}\right)=\left(r\left(x_{i}, x_{j}\right)\right)[N]
$$

Remark 9.8. Clearly the left action induced by $r[N]$ satisfies

$$
{ }^{(x[N])}(y[N])=\left({ }^{x} y\right)[N]
$$

So $\mathbf{1 1}$ is satisfied and therefore $(Y[N], r[N])$ is a square-free solution. There is an isomorphism of solutions

$$
\varphi_{N}:(Y, r) \longrightarrow(Y[N], r[N]) \quad x_{i} \mapsto x_{i+N} .
$$

Furthermore,

$$
\mathcal{G}(Y[N], r[N])=\mathcal{G}(Y, r)[N]={ }_{g r}\left\langle\mathcal{L}_{x}[N] \mid x \in Y\right\rangle .
$$

Definition 9.9. For $m=0,1,2 \cdots$ define a sequence of cycles $\sigma_{m} \in \operatorname{Sym} \mathbb{X}$, each of length $2^{m}$ as follows

$$
\begin{aligned}
& \sigma_{1}=\left(x_{1} x_{2}\right) \\
& \sigma_{2}=\sigma_{1} \vee\left(\sigma_{1}[2]\right)=\left(\begin{array}{llll}
x_{1} & x_{3} & x_{2} & x_{4}
\end{array}\right) \\
& \sigma_{3}=\sigma_{2} \vee\left(\sigma_{2}[4]\right) \\
& \sigma_{m+1}=\sigma_{m} \vee\left(\sigma_{m}\left[2^{m}\right]\right)
\end{aligned}
$$


For $m=0,1,2, \cdots$ we define the solutions $\left(X_{m}, r_{m}\right)$ and $\left(Y_{m}, r_{Y_{m}}\right)$ recursively, as follows.

Clearly $\mathbb{X}=\bigcup_{0 \leq m} X_{m}$.

Definition 9.10. Define the map

$$
r_{\mathbb{X}}: \mathbb{X} \times \mathbb{X} \longrightarrow \mathbb{X} \times \mathbb{X} \quad r(x, y)=r_{m}(x, y) \text { where } x, y \in X_{m}
$$

Theorem 9.11. In assumption and notation as above Let

$$
\left(X_{0}, r_{0}\right),\left(X_{1}, r_{1}\right), \cdots,\left(X_{m}, r_{m}\right), \cdots
$$

be the infinite sequence of quadratic sets defined in Definition 9.9 . Then the following conditions hold for each $m=0,1,2, \ldots$ :

(1) $\left(X_{m}, r_{m}\right)$ is a square-free solution of order $\left|X_{m}\right|=2^{m-1}+1$

(2) $\left(X_{m+1}, r_{m+1}\right)$ is an extension of $\left(X_{m}, r_{m}\right)$.

(3) $\operatorname{Ret}\left(X_{m+1}, r_{m+1}\right) \approx\left(X_{m}, r_{m}\right)$. (i.e. $\left(X_{m+1}, r_{m+1}\right)$ is a blow up of $\left(X_{m}, r_{m}\right)$.

(4) $\operatorname{mpl}\left(X_{m}, r_{m}\right)=m, m=0,1,2 \cdots$.

(5) Each group $\mathcal{G}_{m+1}=\mathcal{G}\left(X_{m+1}, r_{m+1}\right)$ is isomorphic to the wreath product $\mathcal{G}\left(X_{m}, r_{m}\right)$ wr $C_{2}$, so $\mathcal{G}_{m+1}=\underbrace{\left(\left(C_{2} \text { wr } C_{2}\right) \mathrm{wr} \cdots\right) \text { wr } C_{2}}_{m \text { times }}$.

(6) There are equalities $\operatorname{sl}\left(G_{m}\right)=m, \operatorname{sl}\left(\mathcal{G}_{m}\right)=m-1$.

(7) $\left(\mathbb{X}, r_{\mathbb{X}}\right)$ is the inverse limit of the solutions $\left(X_{m}, r_{m}\right)$. For the retracts one has $\operatorname{Ret}^{m}\left(\mathbb{X}, r_{\mathbb{X}}\right) \neq \operatorname{Ret}^{m+1}\left(\mathbb{X}, r_{\mathbb{X}}\right)$, and $\operatorname{mpl}(\mathbb{X}, r)=\infty$. Furthermore, the group $\mathcal{G}\left(\mathbb{X}, r_{\mathbb{X}}\right)$ acts nontransitively on $\mathbb{X}$.

Proof. Under the hypothesis of the theorem we prove first several preliminary statements.

Remark 9.12. Let $m$ be an integer, $m \geq 2, N_{m}=2^{m}$

(1) $\left|Y_{m}\right|=2^{m}$ and $\left|X_{m+1}\right|=2^{m}+1$. 
(2) $\sigma_{m}$ is well defined via $\sigma_{k}, k \leq m-1$ and the shift $y \mapsto y\left[N_{m-1}\right]$. We have the following explicit formulae:

$$
\begin{gathered}
\left(\sigma_{m-1}\left[N_{m-1}\right]\right)\left(y\left[N_{m-1}\right]\right)=\left(\sigma_{m-1}(y)\left[N_{m-1}\right] \quad \forall y \in Y_{m-1}\right. \\
\sigma_{m}(y) \quad=y\left[N_{m-1}\right] \in Y_{m-1}\left[N_{m-1}\right], \quad \forall y \in Y_{m-1} \\
\sigma_{m}\left(y\left[N_{m-1}\right]\right)=\sigma_{m-1} y \in Y_{m-1}, \quad \forall y \in Y_{m-1} \\
x\left[N_{m-1}\right] z\left[N_{m-1}\right]=\left({ }^{x} z\right)\left[N_{m-1}\right]
\end{gathered}
$$

Lemma 9.13. For each integer $m \geq 1,\left(Y_{m}, r_{Y_{m}}\right)$ is a square-free solution of order $N_{m}=2^{m}$ and $\sigma_{m} \in \operatorname{Aut}\left(Y_{m}, r_{Y_{m}}\right)$,

Proof. We shall prove the lemma using induction on $m$. Base for the induction, $m=1$. By definition $\left(Y_{1}, r_{Y_{1}}\right)$ is the trivial solution $\left\{x_{1}, x_{2}\right\}$ and clearly $\sigma_{1}=$ $\left(x_{1} x_{2}\right) \in \operatorname{Aut}\left(Y_{1}, r_{Y_{1}}\right)$. Assume now that the lemma is true for all $k \leq m$.

Clearly, by Definition of as a set $Y_{m+1}$ is a disjoint union $Y_{m+1}=Y_{m} \cup Y_{m}\left[N_{m}\right]$, so $\left|Y_{m+1}\right|=2\left|Y_{m}\right|=2^{m+1}$. Furthermore (by definition), the quadratic set $\left(Y_{m+1}, r_{m+1}\right)$ with $r=r_{Y_{m+1}}$ satisfies

$r(x, y)=\left(\sigma_{m}\left[N_{m}\right](y), \sigma_{m}^{-1} x\right) \quad r(y, x)=\left(\sigma_{(} x\right), \sigma_{m}\left[N_{m}\right]^{-1}(y) \quad \forall x \in Y_{m}, y \in Y_{m}\left[N_{m}\right]$.

By assumption $\sigma_{m} \in \operatorname{Aut}\left(Y_{m}, r_{Y_{m}}\right)$, hence $\sigma_{m}\left[N_{m}\right] \in \operatorname{Aut}\left(Y_{m}\left[N_{m}\right], r_{Y_{m}}\left[N_{m}\right]\right)$, Hence by Lemma $8.5\left(Y_{m+1}, r_{m+1}\right)$ is a solution.

We shall now prove that $\sigma_{m+1} \in$ Aut $Y_{m+1}$. By Lemma 3.33 ) it will be enough to show that for each $x \in Y_{m}$

$$
\sigma_{m+1} \circ \mathcal{L}_{x}=\mathcal{L}_{\sigma_{m+1}(x)} \circ \sigma_{m+1}
$$

is an equality of maps in $Y_{m+1}$, or equivalently

$$
\sigma_{m+1}\left({ }^{x} z\right)={ }^{\sigma_{m+1}(x)} \sigma_{m+1}(z) \forall z \in Y_{m+1} .
$$

By the inductive assumption we have

$$
\sigma_{m}\left({ }^{x} z\right)={ }^{\sigma_{m}(x)} \sigma_{m}(z) \forall x, z \in Y_{m} .
$$

Let $x, z \in Y_{m+1}$. By definition $Y_{m+1}=Y_{m} \bigcup Y_{m}\left[N_{m}\right]$ (this is a disjoint union).

Case 1. $x \in Y_{m}$. 1.a. $z \in Y_{m}$

$$
\begin{aligned}
& x z \in Y_{m} \quad \sigma_{m}\left({ }^{x} z\right)={ }^{x} z\left[N_{m}\right] \quad \text { by } \\
& \sigma_{m}(x)=x\left[N_{m}\right] \quad \sigma_{m}(z)=z\left[N_{m}\right] \quad \text { by } \\
& { }^{\sigma_{m}(x)} \sigma_{m}(z)={ }^{x\left[N_{m}\right]} z\left[N_{m}\right]={ }^{x} z\left[N_{m}\right] \text { by }
\end{aligned}
$$


So in this case (9.8) holds. 1.b. $z=y\left[N_{m}\right]$, where $y \in Y_{m}$.

$$
\begin{aligned}
& x z=\left(\sigma_{m}\left[N_{m}\right]\right)(z)=\sigma_{m}(y)\left[N_{m}\right] \in Y_{m}\left[N_{m}\right] \\
& \sigma_{m}\left({ }^{x} z\right)=\sigma_{m}\left(\sigma_{m}(y)\left[N_{m}\right]\right)=\left(\sigma_{m}\right)^{2}(y) \\
& \sigma_{m}(x)=x\left[N_{m}\right] \quad \sigma_{m}(z)=\sigma_{m}\left(y\left[N_{m}\right]\right)=\sigma_{m}(y) \text { by } \\
& \sigma_{m}(x) \sigma_{m}(z)={ }^{x\left[N_{m}\right]} \sigma_{m}(y)=\sigma_{m}\left(\sigma_{m}(y)\right) \quad \text { by }
\end{aligned}
$$

Hence (9.8) holds.

Case 2. $x=\xi\left[N_{m}\right] \in Y_{m}\left[N_{m}\right]$. 2.a. $z \in Y_{m}$ In this case (9.8) follows from the equalities

$$
\begin{array}{ll}
{ }^{x} z=\sigma_{m}(z)=\in Y_{m} & \text { by } \\
\sigma_{m}\left({ }^{x} z\right)=\sigma_{m}\left(\sigma_{m}(z)\right)=\sigma_{m}(z)\left[N_{m}\right] & \text { by } \\
\sigma_{m}(x)=\sigma_{m}\left(\xi\left[N_{m}\right]\right)=\sigma_{m}(\xi) \in Y_{m} & \sigma_{m}(z)=z\left[N_{m}\right] \in Y_{m}\left[N_{m}\right] \\
\sigma_{m}(x) & \text { by } \\
\quad \sigma_{m}(z)={ }^{\sigma_{m}(\xi)} z\left[N_{m}\right]=\sigma_{m}\left[N_{m}\right]\left(z\left[N_{m}\right]\right) & \text { by } \\
\quad=\left(\sigma_{m}(z)\right)\left[N_{m}\right] & \text { by }
\end{array}
$$

2.b. $z=y\left[N_{m}\right], y \in Y_{m}$. Note that

$$
\begin{aligned}
& { }^{x} z={ }^{\xi}\left[N_{m}\right] y\left[N_{m}\right]=\left({ }^{\xi} y\right)\left[N_{m}\right] \in Y_{m}\left[N_{m}\right] \quad \text { by } \\
& \sigma_{m}\left({ }^{x} z\right)=\sigma_{m}\left(\left({ }^{\xi} y\right)\left[N_{m}\right]\right)=\sigma_{m}\left({ }^{\xi} y\right) \quad \text { by } \\
& \sigma_{m}(x)=\sigma_{m}(\xi) \in Y_{m} \quad \sigma_{m}(z)=\sigma_{m}\left(y\left[N_{m}\right]\right)=\sigma_{m}(y) \in Y_{m} \quad \text { by } \quad \text { (9.5) } \\
& { }^{\sigma_{m}(x)} \sigma_{m}(z)={ }^{\sigma_{m}(\xi)} \sigma_{m}(y)=\sigma_{m}\left(\xi_{y} \quad\right. \text { by IH and (9.8). }
\end{aligned}
$$

where IH denotes the inductive hypothesis.

We have shown that $\sigma_{m+1} \in$ Aut $Y_{m+1}$, which verifies the lemma.

The following corollary is straightforward from the recursive definition of the quadratic sets $\left(X_{m}, r_{m}\right)$ and Lemma 8.5 .

Corollary 9.14. For each $m=0,1,2, \cdots,\left(X_{m}, r_{m}\right)$ is a square-free solution, of order $\left|X_{m}\right|=2^{m-1}+1$. Furthermore, $\left(X_{m+1}, r_{m+1}\right)$ is an extension of $\left(X_{m}, r_{m}\right)$.

The following lemma gives explicit recursive presentation of the left actions in $X_{m}$, respectively $Y_{m}$

Lemma 9.15. Let $m \geq 2$. 
(1) Let $x \in X_{m+1}$ then following equalities hold.

$$
\begin{aligned}
\forall x \in Y_{m-1} & \mathcal{L}_{x \mid Y_{m}}=\mathcal{L}_{x \mid Y_{m-1}} \circ \sigma_{m-1}\left[N_{m-1}\right] \\
& \mathcal{L}_{x \mid X_{m+1}}=\mathcal{L}_{x \mid Y_{m}}
\end{aligned}
$$

$\forall y \in Y_{m-1}\left[N_{m-1}\right], y=x\left[N_{m-1}\right], x \in Y_{m-1} \quad \mathcal{L}_{y \mid Y_{m}}=\left(\mathcal{L}_{x \mid Y_{m-1}}\right)\left[N_{m-1}\right] \circ \sigma_{m-1}$

$$
\begin{gathered}
\mathcal{L}_{y \mid X_{m+1}}=\mathcal{L}_{y \mid Y_{m}} \\
z=x_{2^{m}+1} \quad \mathcal{L} z \mid X_{m+1}=\sigma_{m} .
\end{gathered}
$$

(2) For all $i, i=2 k-1,1 \leq k \leq 2^{m-1}$ one has

$$
\mathcal{L}_{x_{i} \mid X_{m+1}}=\mathcal{L}_{x_{i+1} \mid X_{m+1}},
$$

$$
\mathcal{L}_{x_{i}} \neq \mathcal{L}_{x_{j}} \quad \text { whenever } \quad j \neq i, i+1, \quad 1 \leq j \leq 2^{m}+1
$$

(3) There is an isomorphism of solutions

$$
\operatorname{Ret}\left(X_{m+1}, r_{m+1}\right) \simeq\left(X_{m}, r_{m}\right) .
$$

Proof. The equalities (9.10) follow from the recursive definition of $Y_{m}$ and $X_{m}, m=$ $0,1,2, \cdots$ see Definition 9.9, (9.3). The recursive definition and (9.10) imply (9.11). Then

$$
\left[X_{m+1}\right]=\left\{\left[x_{1}\right],\left[x_{3}\right], \cdots\left[x_{2^{m-2}+1}\right], \cdots\left[x_{2^{m-1}-1}\right]\right\} .
$$

It is easy to see that $\left(\left[X_{m+1}\right], r_{\left[X_{m+1}\right]}\right) \simeq\left(X_{m}, r_{m}\right)$.

Now the statement of the theorem follows easily. Indeed, Corollary 9.14 verifies parts (11), and (2). Part (3) follows from Lemma 9.15, Clearly, induction on $m$ and (3i) straightforwardly imply (4). (5) is clear from the construction. (5D) implies $\operatorname{sl}\left(\mathcal{G}_{m}\right)=m-1$. Hence, by Theorem 7.25 we have $\operatorname{sl}\left(G_{m}\right)=\operatorname{sl}\left(\mathcal{G}_{m}\right)+1$, which proves (6). Finally, (7) is clear.

Construction 9.16. Let $R$ be a finite ring and $A$ a finite faithful $R$-module. Let $\omega$ be a fixed unit in $R$. For $a \in A$, let $L_{a}$ be the permutation $x \mapsto \omega x+(1-\omega) a$.

Note: We do not need to assume that $R$ is commutative, since we work only in the subring generated by 1 and $\omega$. Also, note that $L_{a}$ has the effect of multiplying $x-a$ by $\omega$; so it is clearly a permutation fixing $a$. (Its inverse is obtained by replacing $\omega$ by its inverse.) If $\omega=-1$, it is the inversion in $a$.

Proposition 9.17. The following are equivalent:

(a) the maps $L_{a}$, for $a \in A$, give a solution;

(b) the maps $L_{a}$, for $a \in A$, all commute;

(c) $(1-\omega)^{2}=0$.

Proof. Clearly $L_{a}(a)=a$ holds for all $a \in A$. Thus, the maps form a solution if and only if

$$
L_{a_{b}} L_{a^{b}}=L_{a} L_{b}
$$


where ${ }^{a} b=L_{a}(b)$ and $a^{b}=L_{b}^{-1}(a)$; and a short calculation (see below) shows that this condition and the condition $L a L_{b}=L_{b} L_{a}$ hold for all $a$ and $b$ if and only if $(1-\omega)^{2}=0$.

If $\omega=1$, then $L_{a}$ is the identity map for all $a$, and the multipermutation level is 1 . Otherwise, $L_{a}=L_{b}$ if and only if $(1-\omega)(a-b)=0$; so the elements of the reduct are the cosets of the submodule $\{a \in A:(1-\omega) a=0$ (the annihilator of $1-\omega$. Since $(1-\omega)^{2}=0$, the element $(1-\omega)$ acts as zero on the quotient module $A / I$, so the reduct has mpl 1, and the original solution has mpl 2 .

Calculations. $L_{a}$ maps $x$ to $\omega x+(1-\omega) a$, and so

$$
\begin{aligned}
L_{a} L_{b} & \mapsto \omega x+(1-\omega) b \\
& \mapsto \omega(\omega x+(1-\omega) b)+(1-\omega) a \\
& =\omega^{2} x+(1-\omega)(a+\omega b) .
\end{aligned}
$$

Similarly $L_{b} L_{a}$ maps $x$ to $\omega^{2} x+(1-\omega)(b+\omega a)$. These are equal if and only if $(1-\omega)^{2}(a-b)=0$. For this to hold for all $a$ and $b$, it is necessary and sufficient that $(1-\omega)^{2}=0$.

Now $a^{b}=\omega^{-1} a+\left(1-\omega^{-1}\right) b$ and ${ }^{a} b=\omega b+(1-\omega) a$, so we calculate that $L_{a_{b}} L_{a^{b}}$ maps $x$ to

$$
\omega^{2} x+(1-\omega)(2-\omega) a+(1-\omega)(2 \omega-1) b .
$$

This is equal to $L_{a} L_{b}(x)$ if and only if $(1-\omega)^{2}(a-b)=0$, and the conclusion follows as before.

\section{More about YB PERmutation Groups}

Let $(Z, r)$ be an YB extension of the disjoint solutions $\left(X, r_{X}\right)$ and $\left(Y, r_{Y}\right)$. Then for every $z \in Z$ the action $\mathcal{L}_{z}$ splits

$$
\mathcal{L}_{z}=\left(\mathcal{L}_{z}\right)_{\mid X} \circ\left(\mathcal{L}_{z}\right)_{\mid X Y} .
$$

Recall that if $\left(X, r_{X}\right)$ and $\left(Y, r_{Y}\right)$ are two disjoint solutions Then the trivial extension $(Z, r)$ is defined as $Z=X \bigcup Y$, with $r(x, \alpha)=(\alpha, x)$, for all $x \in X, \alpha \in Y$.

Proposition 10.1. Let $\left(X, r_{X}\right)$ and $\left(Y, r_{Y}\right)$ be disjoint square-free solutions with $G_{1}=G\left(X, r_{X}\right), G_{2}=\left(Y, r_{Y}\right), \mathcal{G}_{1}=\mathcal{G}\left(X, r_{X}\right), \mathcal{G}_{2}=\mathcal{G}\left(Y, r_{Y}\right)$. Then the following conditions hold.

(1) Suppose $(Z, r)$ is the trivial extension of $\left(X, r_{X}\right)$ and $\left(Y, r_{Y}\right)$. Then it is a square-free solution, and

$$
G(Z, r)=G_{1} \times G_{2} \quad \mathcal{G}(Z, r)=\mathcal{G}_{1} \times \mathcal{G}_{2}
$$

Conversely, if $G_{1}$ and $G_{2}$ ate permutation $Y B$ groups, then $G=G_{1} \times G_{2}$ is a permutation $Y B$ group. 
(2) Suppose $(Z, r)$ is an involutive extension which satisfies

$$
r(\alpha, x)=\left({ }^{\alpha} x, \alpha\right) \quad \forall x \in X, \alpha \in Y .
$$

Then $(Z, r)$ is a solution if and only if the assignment $\alpha \mapsto\left(\mathcal{L}_{\alpha}\right)_{\mid X}$ extends to a homomorphism

$$
G\left(Y, r_{Y}\right) \longrightarrow \operatorname{Aut}\left(X, r_{X}\right)
$$

In other words, $G\left(Y, r_{Y}\right)$ acts as automorphisms on $(X, r)$. In this case $G=G(Z, r)$ and the $Y B$ permutation group $\mathcal{G}=\mathcal{G}(Z, r)$ are semidirect products:

$$
G=G_{1} \rtimes G_{2}, \quad \mathcal{G}=\mathcal{G}_{1} \rtimes \mathcal{G}_{2} .
$$

(3) Conversely, suppose there is an action of $G\left(Y, r_{Y}\right)$ on $G\left(X, r_{X}\right)$, such that $X$ is invariant under this action. Then the formula (10.1) induces canonically a solution $(Z, r)$ on $Z$. In particular, a semidirect product $\mathcal{G}_{1} \rtimes \mathcal{G}_{2}$ of two permutation $Y B$ groups defined via an action of $G\left(Y, r_{Y}\right)$ on $G\left(X, r_{X}\right)$, which keeps $X$ invariant is itself a permutation $Y B$ group.

(4) Suppose $(Z, r)$ is the wreath product of solutions $(Z, r)=\left(X, r_{X}\right) \mathrm{wr}\left(Y, r_{Y}\right)$, see Definition 8.6. Then there are equalities

$$
G(Z, r)=G_{1} \text { wr } G_{2} \quad \mathcal{G}(Z, r)=\mathcal{G}_{1} \text { wr } \mathcal{G}_{2}
$$

Question 10.2. Under what conditions is the wreath product $G_{1}$ wr $G_{2}$ of two permutation $Y B$ groups $G_{1}$ and $G_{2}$ ismorphic to a permutation $Y B$ group?

Acknowledgements. The work was completed while the authors were attending the Isaac Newton Institute Programme on Combinatorics and Statistical Mechanics (CSM) 2008. They thank the Isaac Newton Institute for local support and for the inspiring working atmosphere. The second author also thanks the ICTP in Trieste, and the University of Granada, for support during some stages of the project.

\section{REFERENCES}

[AS] Artin, M. And Schelter, W., Graded algebras of global dimension 3, Adv. Math. 66 (1987), 171-216.

[C] Peter J. Cameron, Permutation Groups, Cambridge University Press 45 (1999) 220pp.

[C08] Cameron, Peter J., Introduction to Algebra, Oxford University Press (2008) 337pp.

[CJR08] Cedó, F., Jespers, E. AND DELRIo, A, Involutive Yang-Baxter groups, arXiv:0803.4054v2 [math.QA] 28 Mar 2008, 19 pp.

[CJR09] Cedó, F., Jespers, E., OKniŃski J., Retractability of set theoretic solutions of the Yang Baxter equation, arXiv:0903.3478v2 [math.GR] 23 Mar 2009, 13 pp.

[Dri] Drinfeld, V., On some unsolved problems in quantum group theory, Lecture Notes in Mathematics 1510 (1992), 1-8.

[ESS] Etingof, P., Schedler, T. And Soloviev, A., Set-theoretical solutions to the quantum Yang-Baxter equation, Duke Math. J. 100 (1999), 169-209.

[GAP] The GaP Group, GAP - Groups, Algorithms, and Programming, Version 4.4.12; 2008. (HTTP://WWW.GAP-SYSTEM.ORG)

[GI94] Gateva-Ivanova, T., Noetherian properties of skew polynomial rings with binomial relations, Trans. Amer. Math. Soc. 343 (1994), 203-219.

[GI96-1] Gateva-Ivanova, T., Skew polynomial rings with binomial relations, J. Algebra 185 (1996), 710-753.

[GI96-2] Gateva-Ivanova, T., Regularity of the skew polynomial rings with binomial relations, Preprint (1996). 
[GI00] Gateva-Ivanova, T., Set theoretic solutions of the Yang-Baxter equation, Mathematics and educhtion in Mathematics, Proc. of the Twenty Ninth Spring Conference of the Union of Bulgarian Mathematicians, Lovetch (2000), 107-117.

[GI04] T. Gateva-Ivanova, Quantum binomial algebras, Artin-Schelter regular rings, and solutions of the Yang-Baxter equations, SERdica Math. J. 30 (2004), 431-470.

[GI] Gateva-Ivanova, T., A combinatorial approach to the set-theoretic solutions of the YangBaxter equation, J.MATH.PHYS., 45 (2004), 3828-3858.

[GI08] Gateva-Ivanova, T.. Set-theoretic solutions of YBE, a combinatorial approach - TalK to the seminar Combinatorics and Statistical Mechanics, Isaac Neuton Institute, MARCH 2008.

[GIM08] T. Gateva-Ivanova, T. And Majid, S., Matched pairs approach to set theoretic solutions of the Yang-Baxter equation, J. Algebra 319 (2008) 1462-1529.

[GIM07] T. Gateva-Ivanova, T. and Majid, S., Set Theoretic Solutions of the Yang-Baxter Equations, Graphs and Computations, J. Symb. Comp. 42 (2007) 1079-1112.

[GIM0806] T. Gateva-IVAnova, T. And Majid, S., Quantum spaces associated to multipermutation solutions of level two, ARXIV:0806.2928v2 [MATH.QA] 23 Jun 2008, 34 PP.

[GB] Gateva-Ivanova, T. and Van den Bergh, M., Semigroups of I-type, J. Algebra 206 (1998), 97-112.

[GJO] Gateva-Ivanova, T., Jespers, E. and Okninski, J., Quadratic algebras of skew polynomial type and underlying semigroups, ARXIV:MATH.RA/0210217 v1; J.AlgEBRA, 270 (2003), 635-659.

[JO] Jespers, E. and Okninski, J., Binomial Semigroups, J. Algebra 202 (1998), 250-275.

[L] G. Laffaille, Quantum binomial algebras, Colloquium on Homology and Representation Theory (Spanish) (Vaquerías, 1998). Bol. Acad. Nac. Cienc. (Córdoba) 65 (2000), 177-182.

[LYZ] J. Lu, M. Yan, Y. Zhu On the set-theoretical Yang-Baxter equation, Duke Math. J. 104 (2000) $1-18$.

[M90] Majid, S., Matched pairs of Lie groups associated to solutions of the YangBaxter equations. Pac. J. Math., 141 (1990) 311-332.

[M95] Majid, S., Foundations of quantum group theory, Cambridge Univ. Press (1995).

[Ma] Manin, Yu., Quantum Groups and Non Commutative Geometry Montreal UniverSITY REPORT NO. CRM- 1561, 1988

[MI] Milne, J.S., Group Theory, v2.11. (2003), HTtP://WWW.JMilne.org/Math/

[Ru] Rump, W., A decomposition theorem for square-free unitary solutions of the quantum YangBaxter equation, AdVANCES in Mathematics, 193 (2005), 40-55.

[RTF] N.Yu. Reshetikhin, L.A. Takhtadzhyan, L.D. Faddeev Quantization of Lie groups and Lie algebras (in Russian), Algebra i Analiz 1 (1989), PP. 178-206; English translation in Leningrad Math.J. 1 (1990), PP. 193-225.

[TA81] M. TAKEUCHI Matched pairs of groups and bismash products of Hopf algebras, Commun. ALG., 9 (1981) 841.

[TA] M. Takeuchi Survey on matched pairs of groups. An elementary approach to the ESS-LYZ theory, Banach Center Publ. 61 (2003) 305-331.

[V] A.P. Veselov Yang-Baxter maps: dynamical point of view, ARXIV:MATH/0612814v1 [MATH.QA]

[WX] A. Weinstein And P. Xu Classical solutions of the quantum Yang-Baxter equation, Comm. Math. Phys. 148 (1992), Pp. 309-343.

TGi: Institute of Mathematics and Informatics, Bulgarian Academy of Sciences, Sofia 1113, Bulgaria, P.C: Queen Mary, University of London, School of Mathematics, Mile END RD, LONDON E1 4NS, UK

E-mail address: tatianagateva@yahoo.com, tatyana@aubg.bg, p.j.cameron@qmul.ac.uk 